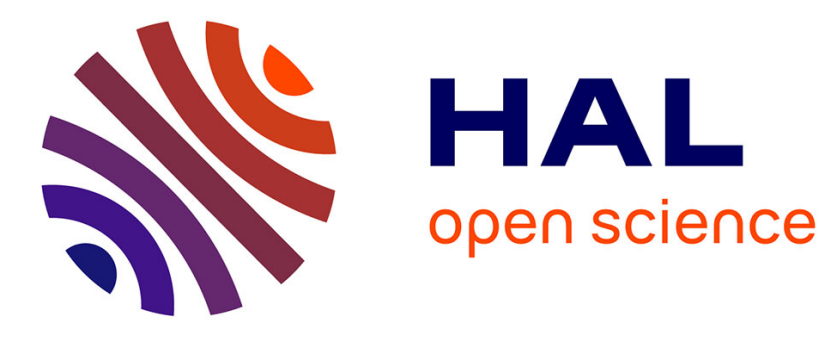

\title{
Fonctions Régulues
}

Goulwen Fichou, Johannes Huisman, Frédéric Mangolte, Jean-Philippe

Monnier

\section{To cite this version:}

Goulwen Fichou, Johannes Huisman, Frédéric Mangolte, Jean-Philippe Monnier. Fonctions Régulues. Journal für die reine und angewandte Mathematik, 2016, 718 (718), pp.103-151. 10.1515/crelle-20140034. hal-00652828v4

\section{HAL Id: hal-00652828 \\ https://hal.science/hal-00652828v4}

Submitted on 28 Mar 2014

HAL is a multi-disciplinary open access archive for the deposit and dissemination of scientific research documents, whether they are published or not. The documents may come from teaching and research institutions in France or abroad, or from public or private research centers.
L'archive ouverte pluridisciplinaire HAL, est destinée au dépôt et à la diffusion de documents scientifiques de niveau recherche, publiés ou non, émanant des établissements d'enseignement et de recherche français ou étrangers, des laboratoires publics ou privés. 


\title{
FONCTIONS RÉGULUES
}

\author{
GOULWEN FICHOU, JOHANNES HUISMAN, FRÉDÉRIC MANGOLTE \\ JEAN-PHILIPPE MONNIER
}

MSC 2000 : 14P99, 14E05, 14F17, 26C15

Keywords : regular function, regulous function, rational function, real algebraic variety

RÉSumé. Nous étudions l'anneau des fonctions rationnelles qui se prolongent par continuité sur $\mathbf{R}^{n}$. Nous établissons plusieurs propriétés algébriques de cet anneau dont un Nullstellensatz fort. Nous étudions les propriétés schématiques associées et montrons une version régulue des Théorèmes $\mathrm{A}$ et $\mathrm{B}$ de Cartan. Nous caractérisons géométriquement les idéaux premiers de cet anneau à travers leurs lieux d'annulation et montrons que les fermés régulus coïncident avec les fermés algébriquement constructibles.

\section{English title and abstract. Regulous functions}

We study the ring of rational functions admitting a continuous extension to the real affine space. We establish several properties of this ring. In particular, we prove a strong Nullstelensatz. We study the scheme theoretic properties and prove regulous versions of Theorems A and B of Cartan. We also give a geometrical characterization of prime ideals of this ring in terms of their zero-locus and relate them to euclidean closed Zariski-constructible sets.

\section{TABLE DES MATIÈRES}

1. Introduction

2. Variétés réelles algébriques et fonctions régulières

3. Fonctions régulues sur les variétés affines 10

4. La topologie $k$-régulue est noethérienne $\quad 15$

5. Nullstellenzatz et Théorèmes A et B 23

6. Ensembles régulument fermés 37

Références 


\section{INTRODUCTION}

Lorsqu'on développe la géométrie réelle algébrique ${ }^{1}$ selon le modèle de la géométrie algébrique complexe, des obstacles techniques apparaissent. L'un des exemples le plus emblématique en étant que les versions algébriques complexes des Théorèmes A et B de Cartan (cf. [30, 2.4]) n'ont pas leurs analogues en géométrie réelle algébrique. Dans la théorie régulière telle que développée dans [6, Chapitre 3], aucun des Théorèmes $\mathrm{A}$ et $\mathrm{B}$ de Cartan n'est valable ${ }^{2}$. De même à travers l'approche Nash de la géométrie réelle algébrique, les Théorèmes $\mathrm{A}$ et $\mathrm{B}$ de Cartan ne sont pas vérifiés ${ }^{3}$. Dans cet article, nous proposons une approche nouvelle de la géométrie réelle algébrique, basée sur les fonction rationnelles continues, et plus généralement les fonctions rationnelles de classe $\mathcal{C}^{k}$, qu'on appellera $k$-régulues. Pour ces fonctions, qui constituent un assouplissement des fonctions régulières lorsque $k<\infty$, les analogues des Théorèmes $\mathrm{A}$ et $\mathrm{B}$ de Cartan deviennent valables (cf. 5.46 et 5.47).

Avant de donner un aperçu des principaux résultats de cet article, il convient de rappeler certaines notions de géométrie réelle algébrique. Suivant un abus courant en géométrie algébrique, nous dirons qu'une fonction rationnelle sur $\mathbf{R}^{n}$ (cf. 2.8 pour une définition formelle) est une fonction à valeurs réelles $f$ définie sur un ouvert de Zariski non vide $U$ de $\mathbf{R}^{n}$ telle qu'il existe des polynômes $p, q \in \mathbf{R}\left[x_{1}, \ldots, x_{n}\right]$ pour lesquels

$$
f(x)=\frac{p(x)}{q(x)}
$$

pour chaque $x \in U$. Cette écriture fractionnaire sous-entend que la fonction polynomiale $q$ ne s'annule pas sur $U$. Deux fonctions rationnelles sur $\mathbf{R}^{n}$ sont alors égales si elles coïncident sur un ouvert de Zariski non vide contenu dans leurs domaines de définition. Une fonction rationnelle $f$ sur $\mathbf{R}^{n}$ possède un plus grand ouvert de Zariski sur lequel elle est définie (cf. [6, Proposition 3.2.3]). Cet ouvert est le domaine de $f$, il est noté $\operatorname{dom}(f)$. Nous noterons $\operatorname{indet}(f)$ le complémentaire du domaine de $f$, c'est le lieu d'indétermination de $f$. A titre d'exemple, le domaine de la fonction rationnelle $f$ sur $\mathbf{R}$ définie par $f(x)=1 / x$ est égal à $\mathbf{R} \backslash\{0\}$ et indet $(f)=\{0\}$. L'ensemble des fonctions rationnelles sur $\mathbf{R}^{n}$ est un corps et s'identifie au corps des fractions rationnelles $\mathbf{R}\left(x_{1}, \ldots, x_{n}\right)$, il peut aussi être noté $\mathbf{R}\left(\mathbf{R}^{n}\right)$.

Une fonction rationnelle $f$ sur $\mathbf{R}^{n}$ est une fonction régulière sur $\mathbf{R}^{n}$ si elle est définie sur $\mathbf{R}^{n}$ tout entier, i.e., si $\operatorname{dom}(f)=\mathbf{R}^{n}$ [6, Définition 3.2.1]. A titre d'exemple, la fonction rationnelle $f(x)=1 /\left(x^{2}+1\right)$ est régulière sur $\mathbf{R}$. L'ensemble des fonctions régulières sur $\mathbf{R}^{n}$ est un sous-anneau du corps des fonctions rationnelles sur $\mathbf{R}^{n}$, que nous noterons $\mathcal{R}^{\infty}\left(\mathbf{R}^{n}\right)$. Nous justifierons plus loin ce choix de notation.

1. Nous ferons la distinction ici entre la «géométrie algébrique réelle» et la «géométrie réelle algébrique»; cette dernière étudie les variétés réelles possédant une structure algébrique, alors que la première étudie les variétés algébriques complexes munies d'une structure réelle.

2. Voir [6, Exemple 12.1.5] pour un contre-exemple au Théorème A, et [8, Theorem 1] pour un contre-exemple au Théorème $\mathrm{B}$ en géométrie réelle régulière.

3. Voir [17] pour des contre-exemples en géométrie réelle Nash. 
Dans ce travail, nous nous proposons donc d'étudier les fonctions rationnelles sur $\mathbf{R}^{n}$ qui s'étendent par continuité à $\mathbf{R}^{n}$ tout entier. Par «continuité» nous entendons ici la continuité par rapport à la topologie euclidienne. Remarquons tout de suite qu'une fonction rationnelle sur $\mathbf{R}^{n}$ étant continue sur son domaine de définition, il s'agit de fonctions qui s'étendent par continuité à leur lieu d'indétermination. Plus précisément, une fonction régulue sur $\mathbf{R}^{n}$ est une fonction à valeurs réelles définie en tout point de $\mathbf{R}^{n}$, qui est continue pour la topologie euclidienne et qui est rationnelle sur $\mathbf{R}^{n}$. A titre d'exemple, la fonction régulière

$$
f(x, y)=\frac{x^{3}}{x^{2}+y^{2}}
$$

sur $\mathbf{R}^{2} \backslash\{0\}$ s'étend par continuité en l'origine et définit donc une fonction régulue sur $\mathbf{R}^{2}$. Son graphe est la toile du célèbre parapluie de Cartan (voir 6.12). L'ensemble des fonctions régulues sur $\mathbf{R}^{n}$ est un sous-anneau du corps $\mathbf{R}\left(\mathbf{R}^{n}\right)$ des fonctions rationnelles sur $\mathbf{R}^{n}$, que nous notons $\mathcal{R}^{0}\left(\mathbf{R}^{n}\right)$. Une fonction régulière sur $\mathbf{R}^{n}$ étant évidemment régulue, nous obtenons une chaîne de sous-anneaux

$$
\mathcal{R}^{\infty}\left(\mathbf{R}^{n}\right) \subseteq \mathcal{R}^{0}\left(\mathbf{R}^{n}\right) \subseteq \mathbf{R}\left(\mathbf{R}^{n}\right) .
$$

Plus généralement, une fonction sur $\mathbf{R}^{n}$ est $k$-régulue, si elle est à la fois régulière sur un ouvert de Zariski non vide, et de classe $\mathcal{C}^{k}$ sur $\mathbf{R}^{n}$. Ici, $k$ désigne un entier surnaturel, i.e., $k$ est ou bien un entier naturel, ou bien $k$ est égal à $\infty$. A titre d'exemple, la fonction régulière

$$
f(x, y)=\frac{x^{3+k}}{x^{2}+y^{2}}
$$

sur $\mathbf{R}^{2} \backslash\{0\}$ s'étend par continuité en l'origine et définit une fonction $k$ régulue sur $\mathbf{R}^{2}$, si $k$ est un entier naturel. Nous démontrons (cf. Théorème 3.3) qu'une fonction $\infty$-régulue sur $\mathbf{R}^{n}$ est nécessairement régulière. Pour $k$ un entier surnaturel, l'ensemble des fonctions $k$-régulues est un sous-anneau du corps $\mathbf{R}\left(\mathbf{R}^{n}\right)$ des fonctions rationnelles sur $\mathbf{R}^{n}$, qui sera noté $\mathcal{R}^{k}\left(\mathbf{R}^{n}\right)$. Remarquons qu'il n'y a pas de conflit de notation ni avec l'anneau des fonctions régulues $\mathcal{R}^{0}\left(\mathbf{R}^{n}\right)$, ni avec l'anneau de fonctions régulières $\mathcal{R}^{\infty}\left(\mathbf{R}^{n}\right)$ introduits ci-dessus. Nous obtenons finalement une chaîne de sous-anneaux

$$
\mathcal{R}^{\infty}\left(\mathbf{R}^{n}\right) \subseteq \cdots \subseteq \mathcal{R}^{2}\left(\mathbf{R}^{n}\right) \subseteq \mathcal{R}^{1}\left(\mathbf{R}^{n}\right) \subseteq \mathcal{R}^{0}\left(\mathbf{R}^{n}\right) \subseteq \mathbf{R}\left(\mathbf{R}^{n}\right) .
$$

Le plus petit de ses sous-anneaux est égal à l'intersection de tous les autres sous-anneaux de la chaîne, i.e.,

$$
\mathcal{R}^{\infty}\left(\mathbf{R}^{n}\right)=\bigcap_{k \in \mathbf{N}} \mathcal{R}^{k}\left(\mathbf{R}^{n}\right) .
$$

Revenons au contenu de cet article. Dans un premier temps nous déterminons les propriétés algébriques de l'anneau $\mathcal{R}^{k}\left(\mathbf{R}^{n}\right)$ des fonctions $k$-régulues sur $\mathbf{R}^{n}$, où $k$ est un entier naturel. Ces anneaux ont été assez peu étudiés; les seules références qui nous sont connues étant [22, 19]. L'anneau $\mathcal{R}^{\infty}\left(\mathbf{R}^{n}\right)$ des fonctions régulières sur $\mathbf{R}^{n}$, en revanche, a attiré beaucoup d'attention [6]. 
Nous montrons que $\mathcal{R}^{k}\left(\mathbf{R}^{n}\right)$ est un anneau non-noethérien pour lequel le Nullstellensatz est valable (5.24). Cela est d'autant plus remarquable que l'intersection de tous ces anneaux, à savoir $\mathcal{R}^{\infty}\left(\mathbf{R}^{n}\right)$, est un anneau noethérien pour lequel le Nullstellensatz n'est pas valable! Une version affaiblie du Nullstellensatz est néanmoins vraie pour $\mathcal{R}^{\infty}\left(\mathbf{R}^{n}\right)$, cette version fait intervenir le radical réel d'un idéal de fonctions régulières $[6, \S 4.4]$. Ce Nullstellensatz réel, bien qu'intéressant en lui-même, ne répare en rien le défaut accablant de l'anneau des fonctions régulières qui est de posséder trop d'idéaux premiers, ce qui ne manque pas de poser des problèmes en géométrie réelle régulière. Nous verrons, en revanche, que la géométrie réelle $k$-régulue, pour $k$ fini, ne pose pas ces problèmes, et, de ce fait, se rapproche plus de la géométrie algébrique sur un corps algébriquement clos que la géométrie réelle régulière.

Malgré le caractère non noethérien de l'anneau $\mathcal{R}^{k}\left(\mathbf{R}^{n}\right)$, son usage reste raisonnable en géométrie algébrique car nous démontrons que son spectre de Zariski Spec $\mathcal{R}^{k}\left(\mathbf{R}^{n}\right)$ est un espace topologique noethérien. De manière équivalente (grâce à la validité du Nullstellensatz justement) l'ensemble $\mathbf{R}^{n}$ muni de la topologie $k$-régulue est un espace topologique noethérien, lorsque $k$ est fini (cf. 4.3). Ici et dans toute la suite de cet article, la «topologie $k$-régulue» est la topologie dont une base de fermés est la collection des sous-ensembles de la forme

$$
\mathcal{Z}(f)=\left\{x \in \mathbf{R}^{n} \mid f(x)=0\right\},
$$

où $f$ est $k$-régulue sur $\mathbf{R}^{n}$. Cette topologie est strictement plus fine que la topologie de Zariski sur $\mathbf{R}^{n}$, lorsque $n \geq 2$. Cette dernière pouvant également être dénommée topologie régulière ou $\infty$-régulue.

Dans un deuxième temps, nous posons les bases de l'étude des variétés régulues abstraites. Nous revenons sur un fibré en droites régulier pathologique sur $\mathbf{R}^{2}$ bien connu qui n'est pas engendré par ses sections régulières globales [6, Example 12.1.5], et montrons que cette pathologie disparait si ce fibré est interprété comme fibré en droites régulu. Nous en déduisons alors la validité des Théorèmes $\mathrm{A}$ et $\mathrm{B}$ de Cartan dans le cadre régulu (5.46 et 5.47).

Nous concluons par un chapitre consacré à la caractérisation géométrique des fermés régulus de $\mathbf{R}^{n}$ et montrons un résultat fondamental : les fermés régulument irréductibles de $\mathbf{R}^{n}$ coïncident avec les sous-ensembles algébriquement constructibles fermés de $\mathbf{R}^{n}$ (Théorème. 6.4). Dans le cas des courbes et des surfaces, nous poussons notre étude et proposons en particulier une relecture régulue des fameux parapluies de Cartan, de Whitney, et d'un parapluie de Kollár. Nous introduisons aussi un nouveau parapluie cornu (6.12).

A notre connaissance, les fonctions régulues ont été étudiées de façon systématique pour la première fois par Kucharz dans [22] (où elles sont appelées continuous rational). Dans son article, Kucharz montre que ce sont les bonnes fonctions pour approcher le plus algébriquement possible les fonctions continues. Il démontre notamment que toute classe d'homotopie d'une application continue entre deux sphères de dimensions quelconques contient une application régulue, là où les applications polynomiales et régulières font défaut!

Dans [19], Kollár étudie les problèmes de restriction et d'extension de fonctions continues rationnelles définies sur une variété réelle algébrique affine. 
Lorsque la variété est lisse, notre notion de fonction régulue (cf. Définition 2.15) coïncide avec la notion de fonction continue rationnelle de Kollár et de Kucharz. Lorsque la variété est singulière, notre notion de fonction régulue coïncide avec ce que Kollár appelle "héréditairement rationnelle continue" (cf. Remarque 5.39). Mentionnons qu'une nouvelle version [20] de l'article [19], en collaboration avec K. J. Nowak contient une version plus forte du théorème d'extension [20, Proposition 10], donnant un contrôle optimal sur la régularité de l'extension, ceci en utilisant des propriétés élémentaires des ensembles semi-algébriques.

Objets naturels, les fonctions régulues apparaissent aussi dans des résultats antérieurs. En 1978, voir [9, p. 369], Kreisel remarque (sans employer le terme "fonction régulue" bien sûr) que le Positivstellensatz de Stengle [32] permet de représenter tout polynôme $f \in \mathbf{R}\left[x_{1}, \ldots, x_{n}\right]$ positif sur $\mathbf{R}^{n}$ comme une somme de carrés de fonctions régulues sur $\mathbf{R}^{n}$.

Il est à noter que l'étude des isomorphismes $\infty$-régulus sur les surfaces a connu récemment des progrès importants, cf. e.g. [4, 15, 21, 5].

Pour être complets, rappelons que sur $\mathbf{C}$, toute fonction rationnelle continue sur une variété normale est régulière. Dans le cas d'une variété singulière générale, l'étude des fonctions rationnelles continues amène aux concepts de semi-normalité et de semi-normalisation, cf. $[2,1]$.

Remerciements. Nous remercions J. Kollár pour nous avoir transmis une version préliminaire de son article, ainsi que S. Cantat, M. Coste, L. Evain, W. Kucharz, K. Kurdyka, D. Naie et A. Parusiński pour l'intérêt précoce qu'ils ont porté à nos travaux et pour leurs suggestions qui ont contribué à améliorer ce texte. Merci aussi à F. Broglia et F. Acquistapace pour nous avoir signalé les références [2] et [1]. La version finale de cet article doit beaucoup au referee dont la lecture attentive et les remarques ont été très constructives.

Ce travail a bénéficié d'un support partiel provenant du contrat ANR "BirPol" ANR-11-JS01-004-01.

\section{VARIÉtÉS RÉELles ALGÉBRIQUes ET FONCTIONS RÉGULIÈRES}

Ensemble des zéros d'une fonction réelle. Soit $n$ un entier naturel. On définit, de manière générale, l'ensemble des zéros et l'ensemble des non zéros d'une fonction réelle sur $\mathbf{R}^{n}$, ou d'un ensemble de fonctions réelles :

Notation 2.1. Soit $f: \mathbf{R}^{n} \rightarrow \mathbf{R}$ une fonction réelle sur $\mathbf{R}^{n}$. On note $\mathcal{Z}(f)$ l'ensemble des zéros de $f$ dans $\mathbf{R}^{n}$, i.e.,

$$
\mathcal{Z}(f)=\left\{x \in \mathbf{R}^{n} \mid f(x)=0\right\} .
$$

L'ensemble des non-zéros de $f$ est

$$
\mathcal{D}(f)=\left\{x \in \mathbf{R}^{n} \mid f(x) \neq 0\right\} .
$$

Soit $E$ un ensemble de fonctions réelles sur $\mathbf{R}^{n}$. On note $\mathcal{Z}(E)$ l'ensemble des zéros communs des fonctions dans $E$, i.e.,

$$
\mathcal{Z}(E)=\bigcap_{f \in E} \mathcal{Z}(f)
$$

On a les propriétés habituelles suivantes : 
Proposition 2.2. Soit $n$ un entier naturel.

(1) $\mathcal{Z}(1)=\emptyset$ et $\mathcal{Z}(\emptyset)=\mathbf{R}^{n}$.

(2) Soit $E_{\alpha}, \alpha \in A$, une collection d'ensembles de fonctions réelles sur $\mathbf{R}^{n}$. Alors

$$
\mathcal{Z}\left(\bigcup_{\alpha \in A} E_{\alpha}\right)=\bigcap_{\alpha \in A} \mathcal{Z}\left(E_{\alpha}\right)
$$

(3) Soient $E_{1}, \ldots, E_{m}$ un nombre fini d'ensembles de fonctions réelles sur $\mathbf{R}^{n}$, où $m$ est un entier naturel. Alors

$$
\mathcal{Z}\left(E_{1} \cdot \ldots \cdot E_{m}\right)=\mathcal{Z}\left(E_{1}\right) \cup \cdots \cup \mathcal{Z}\left(E_{m}\right) .
$$

(4) Soit $E$ un sous-ensemble d'un anneau $A$ de fonctions réelles sur $\mathbf{R}^{n}$. Alors $\mathcal{Z}(E)=\mathcal{Z}(I)$, où $I$ est l'idéal de $A$ engendré par $E$.

Un propriété moins habituelle, mais cruciale en géométrie réelle, est la suivante :

Proposition 2.3. Soit $n$ un entier naturel. Soit $A$ un anneau de fonctions réelles sur $\mathbf{R}^{n}$, et soient $f_{1}, \ldots, f_{m}$ un nombre fini d'éléments de A. Alors, il existe une fonction réelle $f \in A$ telle que

$$
\mathcal{Z}(f)=\mathcal{Z}\left(f_{1}, \ldots, f_{m}\right)
$$

Plus précisément, la fonction $f=f_{1}^{2}+\cdots+f_{m}^{2}$ convient.

Rappelons qu'un sous-ensemble $F$ de $\mathbf{R}^{n}$ est un fermé de Zariski, s'il existe un sous-ensemble $E$ de l'anneau des fonctions polynomiales réelles sur $\mathbf{R}^{n}$ tel que

$$
\mathcal{Z}(E)=F
$$

Comme ce dernier anneau est isomorphe à l'anneau noethérien des polynômes réels $\mathbf{R}\left[x_{1}, \ldots, x_{n}\right]$, un sous-ensemble $F$ de $\mathbf{R}^{n}$ est un fermé de Zariski si et seulement s'il existe un nombre fini de fonctions polynomiales $f_{1}, \ldots, f_{m}$ sur $\mathbf{R}^{n}$ telles que

$$
\mathcal{Z}\left(f_{1}, \ldots, f_{m}\right)=F
$$

En appliquant la proposition précédente 2.3, on pourra encore dire qu'un sousensemble $F$ de $\mathbf{R}^{n}$ est un fermé de Zariski si et seulement s'il existe une fonction polynomiale $f$ sur $\mathbf{R}^{n}$ telle que

$$
\mathcal{Z}(f)=F
$$

Les fermés de Zariski de $\mathbf{R}^{n}$ constituent la collection des fermés d'une topologie sur $\mathbf{R}^{n}$, la topologie de Zariski sur $\mathbf{R}^{n}$.

Fonctions régulières sur $\mathbf{R}^{n}$. Soit $n$ un entier naturel, et soit $U$ un ouvert de Zariski de $\mathbf{R}^{n}$. Soit $f$ une fonction réelle sur $U$, et $x \in U$. La fonction $f$ est régulière en $x$ s'il existe un voisinage Zariski ouvert $V$ de $x$ dans $U$ et des fonctions polynomiales $p$ et $q$ sur $\mathbf{R}^{n}$ tel que $f(y)=p(y) / q(y)$ pour tout $y \in V$, où il est sous-entendu que $q$ ne s'annule pas sur $V$. La fonction $f$ est régulière sur $U$ si elle est régulière en tout point de $U$. On note $\mathcal{Q}(U)$ l'ensemble des fonctions régulières sur $U$. Cet ensemble est une algèbre réelle de manière évidente. 
Soit $V$ un ouvert de Zariski contenu dans $U$, la restriction à $V$ d'une fonction régulière sur $U$ est régulière sur $V$. Il s'ensuit que $\mathcal{Q}$ est un préfaisceau d'algèbres réelles sur $\mathbf{R}^{n}$. Compte tenu de la définition locale d'une fonction régulière, il est évident que $\mathcal{Q}$ est un faisceau sur $\mathbf{R}^{n}$.

Soit $x$ un point de $\mathbf{R}^{n}$. La fibre $\mathcal{Q}_{x}$ s'identifie avec l'algèbre réelle locale des fonctions rationnelles $p / q$ définies en $x$, i.e., $q(x) \neq 0$. Le faisceau $\mathcal{Q}$ sur $\mathbf{R}^{n}$ est donc un faisceau en algèbres réelles locales.

Il est remarquable que les sections du faisceau $\mathcal{Q}$ au-dessus d'un ouvert de Zariski $U$ de $\mathbf{R}^{n}$ admettent une description globale (cf. [6, Proposition 3.2.3]) :

Proposition 2.4. Soit $n$ un entier naturel, et soit $U$ un ouvert de Zariski de $\mathbf{R}^{n}$. Soit $f$ une fonction réelle sur $U$. Alors $f$ est régulière sur $U$ si et seulement s'il existe des fonctions polynomiales $p$ et $q$ sur $\mathbf{R}^{n}$ telles que $f(x)=$ $p(x) / q(x)$, avec $q(x) \neq 0$, pour tout $x \in U$.

Démonstration. Voir Proposition 2.5 ci-dessous où on démontre l'énoncé dans un cadre plus général.

Variétés réelles algébriques affines. Soit $n$ un entier naturel, et soit $F$ un fermé de Zariski de $\mathbf{R}^{n}$. On considère $F$ muni de la topologie induite par la topologie de Zariski, qu'on appelle topologie de Zariski sur $F$. Notons que cette topologie est encore noethérienne.

Soit $\mathcal{I}$ le faisceau d'idéaux de $\mathcal{Q}$ des fonctions régulières s'annulant sur $F$. Le faisceau quotient $\mathcal{Q} / \mathcal{I}$ sera noté $\mathcal{Q}_{F}$. Son support est égal à $F$. De ce fait, on considère $\mathcal{Q}_{F}$ comme un faisceau sur $F$. C'est un faisceau en algèbres réelles locales de corps résiduel $\mathbf{R}$. On peut donc le considérer comme un sous-faisceau des fonctions réelles sur $F$, et on l'appelle le faisceau des fonctions régulières sur $F$.

On a à nouveau une description globale des sections de $\mathcal{Q}_{F}$ (cf. [6, Proposition 3.2.3]) :

Proposition 2.5. Soit $n$ un entier naturel, et soit $F$ un fermé de Zariski de $\mathbf{R}^{n}$. Soit $U$ un ouvert de Zariski de $F$ et soit $f$ une fonction réelle sur $U$. Alors $f$ est régulière sur $U$ si et seulement s'il existe des fonctions polynomiales $p$ et $q$ sur $\mathbf{R}^{n}$ telles que $f(x)=p(x) / q(x)$, avec $q(x) \neq 0$, pour tout $x \in U$.

Pour la commodité du lecteur, on reproduit la démonstration de [6, Proposition 3.2.3].

Démonstration. Il suffit de démontrer l'implication directe. Supposons donc que $f$ est régulière sur $U$. Comme la topologie de Zariski sur $F$ est noethérienne, il existe un recouvrement ouvert fini $\left\{U_{1}, \ldots, U_{m}\right\}$ de $U$ et des fonctions polynomiales $p_{1}, q_{1}, \ldots, p_{m}, q_{m}$ sur $\mathbf{R}^{n}$ telles que $f_{i}(x)=p_{i}(x) / q_{i}(x)$ pour tout $x \in U_{i}$, pour $i=1, \ldots, m$. Soit $s_{i}$ une fonction polynomiale sur $\mathbf{R}^{n}$ avec $\mathcal{Z}\left(s_{i}\right)=F \backslash U_{i}$, pour tout $i$. Montrons que

sur $U$.

$$
f=\frac{s_{1}^{2} p_{1} q_{1}+\cdots+s_{m}^{2} p_{m} q_{m}}{s_{1}^{2} q_{1}^{2}+\cdots+s_{m}^{2} q_{m}^{2}}
$$

Montrons d'abord que le second membre, qu'on notera $g$ dans la suite, est bien une fonction régulière sur $U$. Soit $x \in U$. Il existe $i$ avec $x \in U_{i}$. On a 
donc $s_{i}^{2}(x) q_{i}^{2}\left(x_{i}\right)>0$. Comme $s_{j}^{2}(x) q_{j}^{2}(x) \geq 0$, pour $j \neq i$, le dénominateur de $g$ ne s'annule pas en $x$. Le second membre est donc bien une fonction régulière sur $U$.

Il reste à montrer que $f=g$ sur $U$. Soit $x \in U$. Soit $I$ l'ensembles des indices $i$ pour lesquels $x \in U_{i}$. Lorsque $i \in I$, on a $f(x)=p_{i}(x) / q_{i}(x)$, et donc aussi

$$
s_{i}^{2}(x) q_{i}^{2} f(x)=s_{i}^{2}(x) p_{i}(x) q_{i}^{2}(x) .
$$

Observons que cette dernière formule est également valable lorsque $i \notin I$ puisque $s_{i}(x)=0$ dans ce cas. Il s'ensuit que

$\left(s_{1}^{2}(x) q_{1}^{2}(x)+\cdots+s_{m}^{2}(x) q_{m}^{2}(x)\right) f(x)=\left(s_{1}^{2}(x) p_{1}(x) q_{1}(x)+\cdots+s_{m}^{2}(x) p_{m}(x) q_{m}(x)\right)$ et donc aussi que $f(x)=g(x)$.

Définition 2.6. Une variété réelle algébrique affine est un espace localement annelé $(X, \mathcal{O})$, où $\mathcal{O}$ est un faisceau en algèbres réelles locales ayant la propriété suivante. Il existe un entier naturel $n$ et un fermé de Zariski $F$ de $\mathbf{R}^{n}$ tels que $\left(F, \mathcal{Q}_{F}\right)$ soit isomorphe à $(X, \mathcal{O})$. $\operatorname{Si}(X, \mathcal{O})$ est une variété réelle algébrique affine, on appellera encore sa topologie la topologie de Zariski sur $X$, et une section de $\mathcal{O}$ sur un ouvert $U$ une fonction régulière sur $U$.

Remarque 2.7. On pourrait penser que cette définition induit une notion naturelle de variété réelle algébrique abstraite, mais l'intérêt d'une telle notion est limité par le fait que tout sous-ensemble localement fermé de $\mathbf{R}^{n}$ (voire de $\mathbf{P}^{n}(\mathbf{R})$ ) muni de son faisceau naturel des fonctions régulières est une variété réelle algébrique affine (voir [6, Proposition 3.2.10], [6, Théorème 3.4.4] et la remarque qui suit).

Définition 2.8. Soit $X$ une variété réelle algébrique affine. Une fonction rationnelle sur $X$ est une classe d'équivalence de paires $(f, U)$, où $U$ est un ouvert dense de $X$ et $f$ est une fonction régulière sur $U$. Deux telles paires $(f, U)$ et $(g, V)$ étant équivalentes s'il existe un ouvert dense $W$ de $X$ contenu dans $U \cap V$ tel que $f_{\mid W}=g_{\mid W}$.

Notons que cette dernière condition est équivalente à la condition

$$
f_{\mid U \cap V}=g_{\mid U \cap V} .
$$

Soit $f$ une fonction rationnelle sur la variété réelle algébrique affine $X$. Considérons l'ensemble $\mathcal{F}$ de toutes les paires $(g, V)$ qui sont équivalentes à $(f, U)$. On introduit une relation d'ordre partiel sur $\mathcal{F}$ en définissant $(g, V) \leq$ $(h, W)$ lorsque $V \subseteq W$. Comme $\mathcal{O}$ est un faisceau, l'ensemble $\mathcal{F}$ contient un plus grand élément $(g, V)$. Comme cet élément est unique, on appelle $V$ le domaine de définition de $f$ et on le note $\operatorname{dom}(f)$. Du coup, on pourra également considérer $f$ comme fonction régulière définie sur $\operatorname{dom}(f)$, son extension rationnelle étant unique.

Proposition 2.9. Soit $X$ une variété réelle algébrique affine et soit $f$ une fonction rationnelle sur $X$. Alors il existe des fonctions régulières $p$ et $q$ sur $X$, avec $q \neq 0$ sur $\operatorname{dom}(f)$, telles que $f=p / q$ sur $\operatorname{dom}(f)$.

Démonstration. Conséquence immédiate de la Proposition 2.5. 
Remarque 2.10. On aurait pu s'attendre à ce que les fonctions $p$ et $q$ ci-dessus soient des fonctions polynomiales sur $X$. La notion de «fonction polynomiale» sur une variété réelle algébrique affine n'as pas de sens intrinsèque. Cette notion dépend du choix d'un plongement de $X$ dans un espace affine $\mathbf{R}^{n}$. Cela dit, si $X$ est un fermé de Zariski de $\mathbf{R}^{n}$, pour un certain entier naturel $n$, on peut supposer que $p$ et $q$ sont des fonctions polynomiales avec les propriétés voulues, comme le montre la démonstration de 2.5 .

Soit $X$ une variété réelle algébrique affine. L'ensemble des fonctions rationnelles sur $X$ est un anneau de manière évidente, l'anneau total des fonctions rationnelles sur $X$. On le note $\mathbf{R}(X)$.

Proposition 2.11. Soit $X$ une variété réelle algébrique affine irréductible. Alors $\mathbf{R}(X)$ est un corps.

Proposition 2.12. Soit $X$ une variété réelle algébrique affine et notons $X_{1}, \ldots, X_{m}$ ses composantes irréductibles. Les morphismes de restriction $\mathbf{R}(X) \rightarrow \mathbf{R}\left(X_{i}\right)$ induisent un isomorphisme d'algèbres réelles

$$
\mathbf{R}(X) \longrightarrow \prod_{i=1}^{m} \mathbf{R}\left(X_{i}\right)
$$

En particulier, l'anneau $\mathbf{R}(X)$ est un produit de $m$ corps.

Variétés réelles algébriques affines lisses et fonctions $k$-régulues. Rappelons la définition d'une variété réelle algébrique lisse (cf. [6, Definition 3.3.9]) :

Définition 2.13. Soit $X$ une variété réelle algébrique affine. La variété $X$ est lisse en un point $x$ de $X$ si l'anneau local $\mathcal{O}_{x}$ est régulier. La variété $X$ est lisse si elle l'est en chacun de ses points.

On rappelle le résultat suivant (cf. [6, Proposition 3.3.10]) :

Proposition 2.14. Soit $X$ une variété réelle algébrique affine et notons $X_{1}, \ldots, X_{m}$ ses composantes irréductibles. Alors, $X$ est lisse si et seulement si

(1) $X$ est réunion disjointe des $X_{i}$, et

(2) chaque variété réelle algébrique affine $X_{i}$ est lisse.

On va étendre la notion de fonction $k$-régulue de l'introduction aux fonctions réelles définies sur une variété réelle algébrique affine lisse. Remarquons que si $X$ est une variété réelle algébrique affine lisse, alors $X$ possède une structure sous-jacente de variété différentiable de classe $\mathcal{C}^{k}$, pour tout entier surnaturel $k$ (cf. [6, Proposition 3.3.6]). La définition suivante est donc naturelle.

Définition 2.15. Soit $k$ un entier surnaturel. Soit $X$ une variété réelle algébrique affine lisse, et soit $f$ une fonction réelle sur $X$. La fonction $f$ est $k$-régulue sur $X$ si

(1) $f$ est de classe $\mathcal{C}^{k}$ sur $X$, et

(2) il existe un ouvert de Zariski $U$ dense dans $X$ tel que $f_{\mid U}$ est régulière. 
On dira qu'une fonction réelle sur $X$ est régulue lorsqu'elle est 0-régulue sur $X$.

On note $\mathcal{R}^{k}(X)$ l'ensemble des fonctions $k$-régulues sur $X$. Cet ensemble est de toute évidence un anneau.

Remarque 2.16. Si $k=0$, la définition ci-dessus a même un sens lorsque la variété réelle algébrique affine est singulière. Pourtant, elle ne donne pas lieu à la bonne notion de fonction régulue sur une telle variété. En effet, Kollár montre qu'une fonction rationnelle sur une variété réelle algébrique affine singulière qui s'étend par continuité à toute la variété peut avoir des propriétés non souhaitables [19, Ex. 2]. Voilà pourquoi on introduit, pour l'instant, uniquement la notion de fonction $k$-régulue sur une variété réelle algébrique affine lisse. Cela nous suffira jusqu'en page 34 où nous reviendrons sur cette définition.

Pour une variété réelle algébrique affine lisse $X$ donnée on dispose d'une chaîne croissante

$$
\mathcal{O}(X) \subseteq \mathcal{R}^{\infty}(X) \subseteq \cdots \subseteq \mathcal{R}^{1}(X) \subseteq \mathcal{R}^{0}(X) .
$$

Le but du paragraphe suivant est de montrer que $\mathcal{O}(X)=\mathcal{R}^{\infty}(X)$.

\section{Fonctions RÉGUlues SUR LES VARIÉtéS AFFines}

Fonctions $\infty$-régulues. Soit $f$ une fonction réelle définie sur $\mathbf{R}^{n}$. Le but de cette section est de démontrer que si $f$ est $\infty$-régulue sur $\mathbf{R}^{n}$, alors elle est régulière sur $\mathbf{R}^{n}$. Cet énoncé est connu (voir par exemple [22, Proposition 2.1]), mais nous allons en donner une démonstration un peu plus détaillée.

On a bien l'inclusion $\mathcal{Q}\left(\mathbf{R}^{n}\right) \subseteq \mathcal{R}^{\infty}\left(\mathbf{R}^{n}\right)$. Le but de cette section est de montrer que cette inclusion est une égalité. Dans le reste de l'article, comme dans l'introduction, il sera ainsi justifié d'utiliser la notation $\mathcal{R}^{\infty}\left(\mathbf{R}^{n}\right)$ pour l'anneau des fonctions régulières sur $\mathbf{R}^{n}$.

Soit $f$ une fonction réelle définie sur $\mathbf{R}^{n}$. Rappelons que $f$ est semialgébrique si son graphe est un sous-ensemble semi-algébrique de $\mathbf{R}^{n} \times \mathbf{R}[6$, Définition 2.2.5]. Rappelons également que $f$ est Nash si $f$ est à la fois semialgébrique et de classe $\mathcal{C}^{\infty}$ [6, Définition 2.9.3]. De manière équivalente, $f$ est Nash si $f$ est une fonction analytique réelle et elle est algébrique sur l'anneau des fonctions régulières sur $\mathbf{R}^{n}$, i.e., il existe des fonctions polynomiales $a_{0}, \ldots, a_{d}$ sur $\mathbf{R}^{n}$ telles que

$$
a_{d} f^{d}+a_{d-1} f^{d-1}+\cdots+a_{0}=0
$$

sur $\mathbf{R}^{n}$, où $a_{d}$ est non identiquement nulle [6, Proposition 8.1.8].

Proposition 3.1 (Kucharz [22]). Soient $n$ un entier naturel et $k$ un entier surnaturel. Une fonction $k$-régulue sur $\mathbf{R}^{n}$ est semi-algébrique. En particulier, une fonction $\infty$-régulue sur $\mathbf{R}^{n}$ est Nash.

Démonstration. Soit $f$ une fonction $k$-régulue sur $\mathbf{R}^{n}$. Notons $U$ le domaine de $f$ comme fonction rationnelle, et soient $p$ et $q$ deux fonctions polynomiales sur $\mathbf{R}^{n}$ telles que $f(x)=p(x) / q(x)$ pour tout $x \in U$. Clairement, le graphe de la restriction de $f$ à $U$ est un sous-ensemble semi-algébrique de $U \times \mathbf{R}$. Comme $f$ est continue, le graphe de la fonction $f$ sur $\mathbf{R}^{n}$ est l'adhérence de 
l'ensemble précédent pour la topologie euclidienne dans $\mathbf{R}^{n+1}$. Or, l'adhérence d'un ensemble semi-algébrique est semi-algébrique [6, Prop. 2.2.2]. Il s'ensuit que $f$ est une fonction semi-algébrique.

Corollaire 3.2. Soient $n$ un entier naturel et $k$ un entier surnaturel. Un sousensemble fermé $k$-régulu de $\mathbf{R}^{n}$ est semi-algébriquement fermé.

Théorème 3.3. Soit $n$ un entier naturel. On a

$$
\mathcal{R}^{\infty}\left(\mathbf{R}^{n}\right)=\mathcal{Q}\left(\mathbf{R}^{n}\right)
$$

i.e., une fonction réelle sur $\mathbf{R}^{n}$ est $\infty$-régulue si et seulement si elle est régulière.

Cet énoncé se généralise certainement au cas où $X$ est une variété réelle algébrique affine lisse mais la preuve en devient plus technique et ne nous semble pas apporter d'idée nouvelle.

Démonstration. Comme une fonction régulière est trivialement $\infty$-régulue, il suffit de démontrer la réciproque.

Soit $U$ le domaine de la fonction rationnelle $f$. Ecrivons $f=p / q$, où $p$ et $q$ sont des fonctions polynomiales sur $\mathbf{R}^{n}, q$ ne s'annulant pas sur $U$. On montre que la fraction rationnelle $p / q$ est définie en tout point de $\mathbf{R}^{n}$. Autrement dit, on montre que, pour tout $x \in \mathbf{R}^{n}$, la fraction rationnelle $p / q$ appartient à l'anneau local $\mathcal{Q}_{x}$ des germes des fonctions régulières en $x$. Il suffit de le montrer pour $x$ l'origine de $\mathbf{R}^{n}$.

D'après la proposition précédente, la fonction $f$ sur $\mathbf{R}^{n}$ est semi-algébrique. Comme $f$ est de classe $\mathcal{C}^{\infty}$, elle est Nash et donc analytique réelle [6, Proposition 8.1.6]. Ainsi $f$ définit un germe d'une fonction analytique réelle en 0 ayant la propriété que $q f=p$. La fonction polynomiale $q$ divise donc $p$ dans l'anneau local des germes des fonctions analytiques réelles en 0. Cela implique que $q$ divise $p$ dans l'anneau des séries formelles $\mathbf{R}\left[\left[x_{1}, \ldots, x_{n}\right]\right]$. Ce dernier est aussi la complétion de l'anneau local des fonctions rationnelles sur $\mathbf{R}^{n}$ définies en 0 . Comme ce dernier anneau est noethérien, $q$ divise $p$ dans $\mathcal{Q}_{0}$ [6, Proposition 8.2.11].

On a donc démontré que $f$ est une section globale du faisceau $\mathcal{Q}$, i.e., $f$ est une fonction régulière sur $\mathbf{R}^{n}$.

La topologie $k$-régulue de $\mathbf{R}^{n}$. Soient $n$ un entier naturel et $k$ un entier surnaturel. Un sous-ensemble $F$ de $\mathbf{R}^{n}$ est un fermé $k$-régulu s'il existe un sous-ensemble $E$ de $\mathcal{R}^{k}\left(\mathbf{R}^{n}\right)$ tel que

$$
\mathcal{Z}(E)=F
$$

Remarquons que $F$ n'est pas a priori le lieu des zéros communs d'un nombre fini de fonctions $k$-régulues. Un sous-ensemble $U$ de $\mathbf{R}^{n}$ est un ouvert $k$-régulu si son complémentaire est un fermé $k$-régulu. Les ouverts $k$-régulus de $\mathbf{R}^{n}$ constituent une topologie sur $\mathbf{R}^{n}$, la topologie $k$-régulue.

Soit $X$ un espace topologique. On rappelle que $X$ est noethérien si et seulement si toute suite décroissante dénombrable $F_{i} \supseteq F_{i+1}, i \in \mathbf{N}$, de sous-ensembles fermés de $X$ est stationnaire. De manière équivalente, tout recouvrement ouvert d'un ouvert de $X$ contient un sous-recouvrement fini. 
Compte tenu du Théorème 3.3, un ouvert $\infty$-régulu de $\mathbf{R}^{n}$ est un ouvert de Zariski, et réciproquement. Comme l'anneau des fonctions polynomiales sur $\mathbf{R}^{n}$ est noethérien, la topologie de Zariski sur $\mathbf{R}^{n}$ est noethérienne. Il s'ensuit que la topologie $\infty$-régulue sur $\mathbf{R}^{n}$ est noethérienne. On verra ci-dessous qu'il en est de même pour la topologie $k$-régulue lorsque $k$ est fini (voir Théorème 4.3).

Si $k \leq k^{\prime}$, la topologie $k$-régulue est plus fine que la topologie $k^{\prime}$-régulue. En particulier, La topologie $k$-régulue est plus fine que la topologie de Zariski sur $\mathbf{R}^{n}$. L'exemple suivant montre que la topologie $k$-régulue est strictement plus fine que la topologie de Zariski, lorsque $k$ est un entier naturel et $n \geq 2$.

Exemple 3.4. Soit $C$ la courbe cubique d'équation $y^{2}=x^{2}(x-1)$ dans $\mathbf{R}^{2}$. Comme le polynôme définissant la courbe $C$ est irréductible dans $\mathbf{R}[x, y]$, le sous-ensemble $C$ de $\mathbf{R}^{2}$ est un fermé de Zariski irréductible. En particulier, la clôture de Zariski de $C \backslash\{O\}$ est égale à $C$.

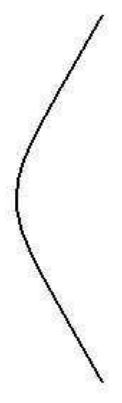

Figure 1. Courbe cubique avec un point isolé.

Soit $f$ la fonction rationnelle sur $\mathbf{R}^{2}$ définie par

$$
f(x, y)=\frac{y^{2}-x^{2}(x-1)}{y^{2}+x^{2}}=1-\frac{x^{3}}{x^{2}+y^{2}} .
$$

Il est clair que $f$ s'étend par continuité en une fonction régulue sur $\mathbf{R}^{2}$. On a $f(O)=1$ et $\mathcal{Z}(f)=C \backslash\{O\}$. Remarquons que l'origine $O$ est un point isolé de la courbe $C$. Par conséquent, $C \backslash\{O\}$ est régulument fermé dans $\mathbf{R}^{2}$, mais non fermé au sens de Zariski. En particulier, le fermé $\infty$-régulu irréductible $C$ n'est pas irréductible pour la topologie régulue sur $\mathbf{R}^{2}$ et s'écrit comme réunion de deux sous-fermés régulus stricts.

$$
C=(C \backslash\{O\}) \cup\{O\} .
$$

Pour montrer que $C \backslash\{O\}$ est un fermé $k$-régulu, pour tout entier naturel $k$, on peut supposer que $k+1$ est impair. Posons

$$
f_{k}=1-(1-f)^{k+1} \text {. }
$$

La fonction $f_{k}$ est bien $k$-régulue sur $\mathbf{R}^{2}$, et, comme $k+1$ est impair, on a $\mathcal{Z}\left(f_{k}\right)=\mathcal{Z}(f)=C \backslash\{O\}$. Cela montre bien que ce dernier ensemble est un fermé $k$-régulu, pour tout entier naturel $k$.

Cet exemple représente en fait le cas général. En effet, on verra dans le Corollaire 6.5 que les topologies $k$-régulue et $k^{\prime}$-régulue coincident pour $k$ et $k^{\prime}$ des entiers naturels quelconques. 
Propriétés élémentaires des fonctions $k$-régulues sur $\mathbf{R}^{n}$. Soit $n$ un entier naturel et $k$ un entier surnaturel. Dans ce paragraphe nous allons étudier les fonctions $k$-régulues sur $\mathbf{R}^{n}$, et les comparer avec d'autres classes de fonctions sur $\mathbf{R}^{n}$.

Dans l'énoncé suivant, et dans le reste de l'article d'ailleurs, on utilise librement la notion de dimension, ou plutôt de codimension, d'un fermé de Zariski de $\mathbf{R}^{n}$, et plus généralement d'un ensemble semi-algébrique de $\mathbf{R}^{n}[6$, $\S 2.8]$.

Proposition 3.5. Soient $n$ un entier naturel et $k$ un entier surnaturel. Soit $f \in \mathcal{R}^{k}\left(\mathbf{R}^{n}\right)$. Soient $p$ et $q$ des fonctions polynomiales sur $\mathbf{R}^{n}$ telles que $f(x)=p(x) / q(x)$ pour chaque $x \in \operatorname{dom}(f)$. Si $p, q$ sont premiers entre eux, alors $\mathcal{Z}(q) \subseteq \mathcal{Z}(p)$ et $\operatorname{codim}_{\mathbf{R}^{n}} \mathcal{Z}(q) \geq 2$.

Démonstration. Il suffit de montrer l'énoncé lorsque $k=0$. Montrons d'abord l'inclusion $\mathcal{Z}(q) \subseteq \mathcal{Z}(p)$. Soit $x \in \mathcal{Z}(q)$. Comme $q$ n'est pas identiquement nulle, l'ensemble de ses zéros $\mathcal{Z}(q)$ est nulle part dense dans $\mathbf{R}^{n}$. Il existe donc une suite $\left(x_{m}\right)$ dans $\mathbf{R}^{n}$ convergeant vers $x$ telle que $q\left(x_{m}\right) \neq 0$, pour tout $k$. On a alors

$$
p(x)=\lim p\left(x_{m}\right)=\lim q\left(x_{m}\right) f\left(x_{m}\right)=q(x) f(x)=0,
$$

i.e., $x \in \mathcal{Z}(p)$.

Montrons ensuite que $\operatorname{codim} \mathcal{Z}(q) \geq 2$. Par l'absurde, supposons que $\operatorname{codim} \mathcal{Z}(q) \leq 1$. Comme $q$ n'est pas identiquement nulle, on a $\operatorname{codim} \mathcal{Z}(q)=$ 1. Il existe donc un diviseur irréductible $q^{\prime}$ de $q$ dans $\mathbf{R}\left[x_{1}, \ldots, x_{n}\right]$ avec $\operatorname{codim} \mathcal{Z}\left(q^{\prime}\right)=1$. D'après ce qui précède, $\mathcal{Z}\left(q^{\prime}\right) \subseteq \mathcal{Z}(p)$. Comme codim $\mathcal{Z}\left(q^{\prime}\right)=$ 1 , on en déduit que $q^{\prime}$ divise $p$ [6, Th. 4.5.1, p.85]. Cela contredit l'hypothèse que $p$ et $q$ sont premiers entre eux.

Comme on a vu, l'énoncé ci-dessus peut être considérablement renforcé lorsque $k=\infty$. En effet, dans ce cas la fonction $f$ est régulière et est de la forme $p / q$ où $q$ ne s'annule pas sur $\mathbf{R}^{n}$, i.e., $\mathcal{Z}(q)=\emptyset$.

Corollaire 3.6. Soient $n \leq 1$ et $k$ un entier surnaturel. Toute fonction $k$ régulue sur $\mathbf{R}^{n}$ est régulière.

Corollaire 3.7. Soit $k$ un entier surnaturel. Une fonction $k$-régulue sur $\mathbf{R}^{2}$ est régulière en dehors d'un ensemble fini.

Corollaire 3.8. Soient $n$ un entier naturel et $k$ un entier surnaturel. Soit $f \in \mathcal{R}^{k}\left(\mathbf{R}^{n}\right)$. Le lieu $\operatorname{indet}(f)$ où $f$ n'est pas une fonction régulière est un fermé de Zariski de $\mathbf{R}^{n}$ de codimension $\geq 2$.

Une application de $\mathbf{R}^{n}$ dans $\mathbf{R}^{m}$ est $k$-régulue lorsque toutes ses fonctions coordonnées le sont. Il n'est pas clair que la composition de deux applications $k$-régulues soit encore $k$-régulue. Un cas où c'est évident est le suivant :

Corollaire 3.9. Soient $\ell, m, n$ des entiers naturels et $k$ un entier surnaturel. Soient

$$
f: \mathbf{R}^{n} \rightarrow \mathbf{R}^{m} \quad \text { et } \quad g: \mathbf{R}^{m} \rightarrow \mathbf{R}^{\ell}
$$

deux applications $k$-régulues. Si l'image de $f$ est de codimension $\leq 1$, alors la composition $g \circ f: \mathbf{R}^{n} \rightarrow \mathbf{R}^{\ell}$ est k-régulue. 
On verra ci-dessous que la conclusion est valable même si la codimension de l'image de $f$ est plus grande que 1 (voir Corollaire 4.14).

Fonctions régulues et fonctions régulières après éclatement. Soit $X$ une variété réelle algébrique affine lisse. Soit $Y$ une sous-variété réelle algébrique lisse de $X$. Soient $f_{0}, \ldots, f_{n}$ des fonctions régulières sur $X$ engendrant l'idéal de $Y$ dans $X$. Notons $f$ l'application régulière de $X \backslash Y$ dans $\mathbf{P}^{n}(\mathbf{R})$ de coordonnées homogènes $f_{0}, \ldots, f_{n}$. L'éclaté $E_{Y}(X)$ de $X$ le long de $Y$ est la clôture de Zariski du graphe de $f$ dans le produit $X \times \mathbf{P}^{n}(\mathbf{R})$. L'éclaté $E_{Y}(X)$ est encore une variété réelle algébrique affine lisse. L'application de projection

$$
\pi: E_{Y}(X) \longrightarrow X
$$

est une application régulière, et birégulière au-dessus de $X \backslash Y$. L'image réciproque de $Y$ par $\pi$ peut s'identifier avec le fibré normal projectif de $Y$ dans $X$.

On aura besoin du résultat suivant :

Théorème 3.10 (Hironaka). Soit $X$ une variété réelle algébrique affine lisse et $f$ une fonction rationnelle sur $X$. Alors il existe une composition finie d'éclatements de centres lisses

$$
\phi: \widetilde{X} \longrightarrow X
$$

telle que l'application rationnelle

$$
f \circ \phi: \widetilde{X} \rightarrow \mathbf{P}^{1}(\mathbf{R})
$$

est régulière sur $\widetilde{X}$ toute entière.

Pour une démonstration, on renvoi le lecteur vers le livre de Kollár [18] (notamment le Corollaire 3.18 amélioré en utilisant Théorème 3.21 au lieu de Théorème 3.17, ceci appliqué à une complexification lisse $X_{\mathbf{C}}$ de $X$ ) .

Soit $X$ une variété réelle algébrique affine lisse. Une fonction réelle $f$ sur $X$ est régulière après éclatements (blow-regular, en anglais) s'il existe une composition d'éclatements à centres lisses $\phi: \tilde{X} \rightarrow X$ telle que la fonction réelle $f \circ \phi$ est régulière. Cette notion est l'analogue algébrique des fonctions qui sont Nash après éclatements ou encore des fonctions analytiques réelles après éclatements [23].

Théorème 3.11. Soit $X$ une variété réelle algébrique affine lisse. Soit $f$ une fonction réelle sur $X$. La fonction $f$ est régulue sur $X$ si et seulement si $f$ est régulière après éclatements. Plus précisément, $f$ est régulue si et seulement s'il existe une composition finie d'éclatements à centres lisses

$$
\phi: \widetilde{X} \longrightarrow X
$$

telle que la composition

$$
f \circ \phi: \widetilde{X} \longrightarrow \mathbf{R}
$$

est régulière. 
Démonstration. Supposons que $f$ est régulière après éclatements, et montrons que $f$ est régulue. Il existe une suite d'éclatements de centres lisses

$$
\phi: \tilde{X} \longrightarrow X
$$

telle que $f \circ \phi$ est régulière. Comme $\phi$ est birationnelle, il existe un ouvert de Zariski, qui est dense dans $X$, sur lequel $f$ est régulière. Il nous reste à montrer que $f$ est continue. Or, la topologie euclidienne sur $X$ est la topologie quotient induite par la topologie euclidienne sur $\tilde{X}$. Le fait que $f \circ \phi$ soit continue implique donc que $f$ est continue. Cela montre bien que $f$ est régulue lorsque $f$ est régulière après éclatements.

Montrons la réciproque. Soit $f$ une fonction régulue sur $X$. Il existe un ouvert de Zariski $U$, dense dans $X$, tel que $f_{\mid U}$ est régulière. En appliquant Théorème 3.10 à $f$, vue comme fonction rationnelle sur $X$, il existe une suite d'éclatements de centres lisses

$$
\phi: \widetilde{X} \longrightarrow X
$$

telle que l'application rationnelle

$$
f \circ \phi: \widetilde{X} \rightarrow \mathbf{P}^{1}(\mathbf{R})
$$

est régulière sur $\tilde{X}$ toute entière. Par définition de composition d'applications rationnelles, cela veut dire que l'application régulière $f_{\mid U} \circ \phi_{\mid \phi^{-1}(U)}$ de $\phi^{-1}(U)$ dans $\mathbf{P}^{1}(\mathbf{R})$ s'étend à $\tilde{X}$ toute entière comme application régulière. Cette extension est nécessairement continue. Or, la composition d'applications continues $f \circ \phi$ est également une extension continue de $f_{\mid U} \circ \phi_{\mid \phi^{-1}(U)}$. Comme $\phi^{-1}(U)$ est dense dans $\tilde{X}$ pour la topologie euclidienne, ces deux extensions sont la même ce qui veut dire que $f \circ \phi$ est régulière, et $f$ est régulière après éclatements.

\section{La topologie $k$-RÉGUlue est noethérienne}

Fonctions régulues et fonctions régulières par strates. Rappelons qu'un sous-ensemble localement fermé de $\mathbf{R}^{n}$, au sens de Zariski, est un sous-ensemble de la forme $U \cap F$, où $F \subseteq \mathbf{R}^{n}$ est un fermé de Zariski et $U \subseteq \mathbf{R}^{n}$ un ouvert de Zariski.

Théorème 4.1. Soient $n$ un entier naturel et $k$ un entier surnaturel. Soit $f$ une fonction $k$-régulue sur $\mathbf{R}^{n}$. Alors, il existe une stratification finie de $\mathbf{R}^{n}$

$$
\mathbf{R}^{n}=\coprod_{i=1}^{m} S_{i}
$$

en sous-ensembles localement fermés de $\mathbf{R}^{n}$ au sens de Zariski telle que la restriction $f_{\mid S_{i}}$ est régulière, pour tout $i$.

Démonstration. Il suffit de traiter le cas $k=0$. On peut, bien-sûr, supposer que $f$ est non identiquement nulle sur $\mathbf{R}^{n}$.

Notons $\mathbf{A}^{n}$ l'espace affine $\operatorname{Spec} \mathbf{R}\left[x_{1}, \ldots, x_{n}\right]$ sur $\mathbf{R}$, de sorte que $\mathbf{A}^{n}(\mathbf{R})=$ $\mathbf{R}^{n}$ et $\mathbf{A}^{n}(\mathbf{C})=\mathbf{C}^{n}$. La conjugaison complexe $\gamma$ agit sur $\mathbf{A}^{n}(\mathbf{C})$. On identifie $\mathbf{A}^{n}(\mathbf{R})$ avec l'ensemble des points fixes de $\gamma$ sur $\mathbf{A}^{n}(\mathbf{C})$. 
Ecrivons $f=r / s$ avec $r$ et $s$ des polynômes réels en $x_{1}, \ldots, x_{n}$. Soit $Y$ le sous-schéma réduit de $\mathbf{A}^{n}$ défini par le polynôme rs. Comme $f$ est non nulle, $Y$ est un sous-schéma de $\mathbf{A}^{n}$ de codimension 1.

Appliquons le théorème de Hironaka ${ }^{4}$ au sous-schéma $Y$ de $\mathbf{A}^{n}$ en éclatant $\mathbf{A}^{n}$, de manière successive, mais seulement en des centres dont l'ensemble des points réels est dense. Plus précisément, il existe une suite finie de morphismes

$$
X_{\ell} \longrightarrow X_{\ell-1} \longrightarrow \cdots \longrightarrow X_{0}=\mathbf{A}^{n}
$$

où chaque morphisme $\pi_{i}: X_{i} \rightarrow X_{i-1}$ est un éclatement de centre lisse $C_{i-1} \subseteq$ $X_{i-1}$ de codimension $\geq 2$ ayant les propriétés suivantes. Pour tout $i$, l'ensemble des points réels $C_{i}(\mathbf{R})$ est dense dans $C_{i}$, et le transformé strict $Y_{\ell}$ de $Y$ dans $X_{\ell}$ n'a que des points réels lisses. Notons $E_{i} \subseteq X_{i}$ le diviseur exceptionnel de $\pi_{i}$. On peut supposer, de plus, que $Y_{\ell}(\mathbf{R})$ intersecte $E_{\ell}(\mathbf{R})$ transversalement en des points réels lisses de $E_{\ell}$. On note encore $\phi$ la composition $\pi_{1} \circ \cdots \circ \pi_{\ell}$. Par construction, $f \circ \phi$ est une fonction rationnelle sur $X_{\ell}$ dont le domaine de définition contient tous les points réels de $X_{\ell}$.

Soit $p$ un point du schéma $\mathbf{A}^{n}$ dont le corps résiduel $\kappa(p)$ est réel. La fibre $\phi^{-1}(p)$ est une réunion finie connexe d'espaces projectifs sur $\kappa(p)$. Soit $P$ l'un de ces espaces projectifs. Comme le domaine de définition de $f \circ \phi$ contient tous les points réels de $X_{\ell}$, la fonction rationnelle $f \circ \phi$ se restreint à une fonction rationnelle sur $P$. Il existe un point $\kappa(p)$-rationnel $q$ de $P$ en lequel $f \circ \phi$ est définie. Soit $F$ le sous-schéma de $X_{0}$ dont le point générique est $p$, et $G$ le sous-schéma de $X_{\ell}$ dont le point générique est $q$. La restriction de $\phi$ à $G$ est un morphisme birationnel dans $F$. Il existe donc des ouverts non vides $U$ de $F$ et $V$ de $G$ tels que $\phi_{\mid V}$ soit un isomorphisme de $V$ sur $U$. Quitte à remplacer $U$ et $V$ par des ouverts plus petits, on peut supposer que $f \circ \phi$ est définie sur $V$. Or $\phi$ induit, sur les points réels, un isomorphisme birégulier de $V(\mathbf{R})$ sur $U(\mathbf{R})$. Il s'ensuit que la restriction de $f$ à $U(\mathbf{R})$ est régulière.

La construction précédente montre que pour tout idéal premier réel $p$ de $\mathbf{R}\left[x_{1}, \ldots, x_{n}\right]$, il existe un sous-ensemble localement fermé irréductible $R_{p}$ dense de $\mathcal{Z}(p)$ tel que $f_{\mid R_{p}}$ est régulière. Il existe alors un nombre fini d'idéaux premiers réels distincts $p_{1}, \ldots, p_{m}$ tels que

$$
\mathbf{R}^{n}=\bigcup_{i=1}^{m} R_{p_{i}}
$$

Pour simplifier la notation, on notera $R_{i}$ au lieu de $R_{p_{i}}$.

On construit ensuite une stratification selon le procédé habituel : en agrandissant la famille $\left\{R_{i}\right\}$ si nécessaire, on peut supposer que toutes les composantes irréductibles des intersections finies des sous-ensembles de la forme $R_{i}$ appartiennent encore à la famille $\left\{R_{i}\right\}$. Introduisons un ordre partiel sur la collection $\left\{R_{i}\right\}$. On pose $R_{i} \leq R_{j}$ si $R_{i}$ est contenu dans la clôture de Zariski $\bar{R}_{j}$ de $R_{j}$. Soit

$$
S_{i}=R_{i} \backslash \bigcup_{R_{j}<R_{i}} \bar{R}_{j}
$$

4. Nous utilisons ici la version [18, Théorème 3.27] 
Le sous-ensemble $S_{i}$ de $\mathbf{R}^{n}$ est localement fermé, et contenu dans $R_{i}$. La restriction de la fonction $f$ à $S_{i}$ est donc bien régulière.

Montrons que $S_{i} \cap S_{j}=\emptyset$ lorsque $i \neq j$. C'est clair lorsque $R_{j}<R_{i}$ ou l'inverse. Supposons donc que $R_{i}$ et $R_{j}$ ne sont pas comparables. Ecrivons l'intersection $R_{i} \cap R_{j}$ comme réunion des $R_{k}$ où $k$ parcourt un ensemble d'indice $I$. Comme on a $R_{k} \subseteq \bar{R}_{i}$ et $R_{k} \subseteq \bar{R}_{j}$, pour tout $k \in I$, on a bien $S_{i} \cap S_{j}=\emptyset$, lorsque $i \neq j$.

Il nous reste à montrer que $\mathbf{R}^{n}=\bigcup S_{i}$. Soit $x \in \mathbf{R}^{n}$ et soit $R_{i}$ le plus petit élément de la collection $\left\{R_{i}\right\}$ tel que $x \in R_{i}$. On a bien $x \in S_{i}$.

Remarque 4.2. Il est possible de prouver le Théorème 4.1, et donc aussi le Théorème 4.3 ci-dessous, sans utiliser le Théorème d'Hironaka; et ce grâce à la Proposition 7 de [20]. Pour cela, on a besoin de la notion de restriction d'une fonction régulue à une sous-variété, cf. Proposition 5.37 et Remarque 5.39.

L'anneau $\mathcal{R}^{\infty}\left(\mathbf{R}^{n}\right)$ est noethérien, la topologie induite est donc noethérienne. En fait c'est encore le cas pour la topologie $k$-régulue, comme on peut le voir en utilisant la noethérianité de la topologie algébriquement constructible $[26,28]$ combinée avec le Théorème 4.1 (qui implique que les fermés $k$-régulus sont algébriquement constructibles, cf. Corollaire 4.12). Nous allons en donner une démonstration directe, en nous inspirant de l'idée donnée dans [27] pour établir la noethérianité de la topologie quasi-analytique. Soit $F=\mathcal{Z}(f)$ avec $f \in \mathcal{R}^{0}\left(\mathbf{R}^{n}\right)$. D'après le Théorème 4.1 , il existe une stratification finie $\mathbf{R}^{n}=\coprod_{i=1}^{m} S_{i}$ en sous-ensembles localement fermés telle que la restriction de $f$ à $S_{i}$ est régulière. On peut même exiger que les strates soient lisses et irréductibles (au sens Zariski, ou encore algébriquement constructible, cf. [28]), et que $F$ soit une réunion de strates. À une telle stratification de $F$, on associe le multi-indice $\mu=\left(\mu_{l}, \mu_{l-1}, \ldots, \mu_{0}\right) \in \mathbf{N}^{l+1}$ où $\mu_{j}$ est le nombre de strates de dimension $j$. On note $\mu_{\mathcal{Z}(f)}$ le plus petit (pour l'ordre lexicographique) des multi-indices associés aux stratifications de $\mathcal{Z}(f)$.

Théorème 4.3. Soient $n$ un entier naturel et $k$ un entier surnaturel. La topologie $k$-régulue sur $\mathbf{R}^{n}$ est noethérienne.

Démonstration. L'anneau $\mathcal{R}^{\infty}\left(\mathbf{R}^{n}\right)$ est noethérien, on peut donc supposer que $k$ est un entier naturel. Il suffit de prouver que la topologie régulue (c'est-à-dire pour $k=0$ ) sur $\mathbf{R}^{n}$ est noethérienne. Tout fermé régulu est une intersection de fermés principaux (un fermé est principal s'il est de la forme $\mathcal{Z}(f)$ pour $f \in \mathcal{R}^{0}\left(\mathbf{R}^{n}\right)$ ). Il suffit donc de montrer que toute suite décroissante de fermés principaux est stationnaire.

On considère une suite décroissante de fermés régulus principaux $\mathcal{Z}\left(f_{\alpha}\right)$, $f_{\alpha} \in \mathcal{R}^{0}\left(\mathbf{R}^{n}\right)$. Il suffit alors de remarquer que $\mu_{\mathcal{Z}(g)}<\mu_{\mathcal{Z}(f)}$ si $f, g \in \mathcal{R}^{0}\left(\mathbf{R}^{n}\right)$ sont telles que $\mathcal{Z}(g)$ est inclus strictement dans $\mathcal{Z}(f)$.

Pour le montrer, prenons une strate $S$ de dimension $s$ pour $\mathcal{Z}(f)$, et supposons que $g$ s'annule sur un sous-ensemble semi-algébrique de dimension $s$ de $S$. Pour une stratification en irréductibles et lisses associée à $\mathcal{Z}(g)$ par le Théorème 4.1, il existe une strate dont l'intersection $U$ avec $S$ est dense dans $S$ au sens de la topologie de Zariski puisque $S$ est irréductible. En particulier $g_{\mid U}$ est régulière et s'annule sur un sous-ensemble semi-algébrique 
de $U$ de dimension maximale, donc $g_{\mid U}$ est identiquement nulle. Par continuité, $g$ s'annule sur l'adhérence de $U$ pour la topologie euclidienne, donc $g$ est nulle sur $S$ par lissité de $S$.

Corollaire 4.4. Soient $n$ un entier naturel et $k$ un entier surnaturel. Tout fermé $k$-régulu de $\mathbf{R}^{n}$ est de la forme $\mathcal{Z}(f)$, pour une certaine fonction $k$ régulue $f$ sur $\mathbf{R}^{n}$.

Démonstration. Ce corollaire se déduit de 4.3 en utilisant la Proposition 2.3.

Corollaire 4.5. Soient $n$ un entier naturel et $k$ un entier surnaturel. Tout fermé $k$-régulu $F$ de $\mathbf{R}^{n}$ est un fermé de Zariski après éclatements, i.e., il existe une composition d'éclatements à centres lisses

$$
\phi: \widetilde{\mathbf{R}}^{n} \longrightarrow \mathbf{R}^{n}
$$

telle que l'image réciproque $\phi^{-1}(F)$ est Zariski fermée dans $\widetilde{\mathbf{R}}^{n}$.

Démonstration. D'après Corollaire 4.4, il existe une fonction $k$-régulue $f$ sur $\mathbf{R}^{n}$ telle que $\mathcal{Z}(f)=F$. D'après Théorème 3.11 , il existe une composition d'éclatements de $\mathbf{R}^{n}$ à centres lisses $\phi$ telle que $f \circ \phi$ est régulière. En particulier,

$$
\mathcal{Z}(f \circ \phi)=\phi^{-1}(\mathcal{Z}(f))=\phi^{-1}(F)
$$

est un fermé de Zariski.

Remarque 4.6. La réciproque du corollaire ci-dessus est fausse comme le prouve l'exemple suivant. Soit $F$ la clôture euclidienne de la partie de dimension $2 \mathrm{du}$ parapluie de Whitney (cf. Exemple 6.12). Après résolution des singularités du parapluie, la préimage de $F$ est la résolution du parapluie complet, qui est un fermé de Zariski.

Même si la topologie $k$-régulue sur $\mathbf{R}^{n}$ est plus fine, $\mathbf{R}^{n}$ reste irréductible pour cette topologie :

Proposition 4.7. Soient $n$ un entier naturel et $k$ un entier surnaturel. Alors $\mathbf{R}^{n}$ muni de la topologie $k$-régulue est irréductible.

Démonstration. Supposons que $\mathbf{R}^{n}=F \cup G$, où $F$ et $G$ sont des fermés $k$ régulus de $\mathbf{R}^{n}$. Il existe des fonctions $k$-régulues $f$ et $g$ sur $\mathbf{R}^{n}$ telles que $\mathcal{Z}(f)=F$ et $\mathcal{Z}(g)=G$. On a

$$
\mathcal{Z}(f g)=\mathcal{Z}(f) \cup \mathcal{Z}(g)=F \cup G=\mathbf{R}^{n} .
$$

Par conséquent, $f g=0$. L'anneau $\mathcal{R}^{k}\left(\mathbf{R}^{n}\right)$ étant un sous-anneau du corps $\mathbf{R}\left(\mathbf{R}^{n}\right)$, il est intègre. On a donc $f=0$ ou $g=0$, i.e., $F=\mathbf{R}^{n}$ ou $G=\mathbf{R}^{n}$.

Ensembles symétriques par arcs. Dans ce paragraphe, nous faisons le lien avec la théorie des ensembles symétriques par arcs introduite par K. Kurdyka. Une fonction semi-algébrique $f: \mathbf{R}^{n} \rightarrow \mathbf{R}$ est appelée analytique par arcs [24] si $f \circ \gamma$ est analytique pour tout arc analytique $\gamma: I \rightarrow \mathbf{R}^{n}$, où $I$ est un intervalle ouvert de $\mathbf{R}$. Il est immédiat que si $f: \mathbf{R}^{n} \rightarrow \mathbf{R}$ devient analytique après une suite finie d'éclatements à centres algébriques lisses bien choisis, alors $f$ est analytique par arcs (Dans [3, Thm. 1.1], Bierstone et Milman montrent en 
fait l'équivalence). Comme une fonction régulière est analytique, on obtient la conséquence suivante du Théorème 3.11, grâce à la Proposition 3.1 :

Corollaire 4.8. Soient $n$ un entier naturel et $k$ un entier surnaturel. Une fonction $k$-régulue sur $\mathbf{R}^{n}$ est analytique par arcs.

Remarque 4.9. Il existe bien évidemment des fonctions analytiques par arcs qui ne sont pas régulues. Par exemple $f(x, y)=\sqrt{x^{4}+y^{4}}$ est analytique par arcs [3] et n'est clairement pas régulue sur $\mathbf{R}^{2}$.

On rappelle qu'un ensemble $E$ semi-algébrique dans $\mathbf{R}^{n}$ est dit symétrique par arcs si et seulement si pour tout arc analytique $\gamma:]-1,1\left[\rightarrow \mathbf{R}^{n}\right.$, si $\gamma(]-1,0[) \subseteq E$ alors $\gamma(]-1,1[) \subseteq E$, voir [24]. Le lien avec les fonctions analytiques par arcs est le suivant. Si $f$ est une fonction analytique par arcs sur $\mathbf{R}^{n}$, alors son lieu de zéros $\mathcal{Z}(f)$ est symétrique par arcs.

Suivant les notations de [24], on appelle $\mathcal{A} \mathcal{R}$ la topologie dont les ensembles fermés sont les ensembles symétriques par arcs. On verra ci-dessous que la topologie $\mathcal{A} \mathcal{R}$ est plus fine que la topologie régulue.

Proposition 4.10. Soient $n$ un entier naturel et $k$ un entier surnaturel. Soit $F$ un fermé $k$-régulu de $\mathbf{R}^{n}$. Alors $F$ est un ensemble symétrique par arcs.

Démonstration. Soit $F=\mathcal{Z}(E)$ un fermé $k$-régulu de $\mathbf{R}^{n}$. D'après le Corollaire $4.8, \mathcal{Z}(f)$ est symétrique par arcs, pour tout $f \in E$. Comme les sousensembles symétriques par arcs sont les fermés d'une topologie,

$$
\mathcal{Z}(E)=\bigcap_{f \in E} \mathcal{Z}(f)
$$

est encore symétrique par arcs.

Remarque 4.11. Le théorème de noethérianité 4.3 est alors un corollaire immédiat du théorème de noethérianité de Kurdyka [24, Th. 1.4.] grâce à la proposition ci-dessus.

Ensembles algébriquement constructibles. Rappelons qu'un sous-ensemble $E$ de $\mathbf{R}^{n}$ est Zariski constructible ou algébriquement constructible s'il est réunion finie de sous-ensembles localement fermés au sens de Zariski.

Corollaire 4.12. Soient $n$ un entier naturel et $k$ un entier surnaturel. Un ensemble fermé $k$-régulu de $\mathbf{R}^{n}$ possède une stratification finie en sous-ensembles localement fermés de $\mathbf{R}^{n}$. En particulier, tout ensemble $k$-régulument fermé de $\mathbf{R}^{n}$ est Zariski constructible.

Notons qu'un sous-ensemble $k$-régulument fermé de $\mathbf{R}^{n}$ n'est pas forcément localement fermé, comme le montre l'exemple suivant.

Exemple 4.13. Soit $C \subseteq \mathbf{R}^{2}$ la courbe cubique ayant la singularité isolée en $O$ de l'Exemple 3.4. Soit $C^{\prime}=C \backslash\{O\}$. Le sous-ensemble

$$
F=\left(C^{\prime} \times \mathbf{R}\right) \cup\{O\}
$$

est un sous-ensemble $k$-régulument fermé de $\mathbf{R}^{3}$ qui n'est pas localement fermé au sens de Zariski. En effet, la clôture de Zariski de $F$ est égale à $C \times \mathbf{R}$. Le sous-ensemble $F$ de $C \times \mathbf{R}$ n'est pas Zariski ouvert pour la topologie induite 
car son intersection avec $\{O\} \times \mathbf{R}$ est réduite à l'origine de $\mathbf{R}^{3}$ et n'est pas ouvert dans $\{O\} \times \mathbf{R}$.

Dans le cas $k=\infty$, l'énoncé du Corollaire 4.12 peut être considérablement renforcé car les fermés sont alors les fermés de Zariski.

Le Théorème 4.1 a encore l'énoncé suivant comme conséquence :

Corollaire 4.14. Soient $\ell, m, n$ des entiers naturels et $k$ un entier surnaturel. Soient

$$
f: \mathbf{R}^{n} \rightarrow \mathbf{R}^{m} \quad \text { et } \quad g: \mathbf{R}^{m} \rightarrow \mathbf{R}^{\ell}
$$

deux applications $k$-régulues. Alors la composition $g \circ f: \mathbf{R}^{n} \rightarrow \mathbf{R}^{\ell}$ est $k$-régulue.

Démonstration. L'application $g \circ f$ est bien-sûr de classe $\mathcal{C}^{k}$. Soit $U$ l'intersection des domaines des fonctions coordonnées de $f$. La restriction de $f$ à $U$ est donc une application régulière dans $\mathbf{R}^{m}$. D'après le Théorème 4.1, il existe une stratification

$$
\mathbf{R}^{m}=\coprod_{i=1}^{p} S_{i}
$$

en sous-ensembles localement fermés de $\mathbf{R}^{m}$ au sens de Zariski telle que la restriction $g_{\mid S_{i}}$ est régulière, pour tout $i$. Soit $i$ tel que $U \cap f^{-1}\left(S_{i}\right)$ est Zariski dense dans $U$. Soit $U^{\prime}$ un ouvert Zariski dense dans $U$ avec $f\left(U^{\prime}\right) \subseteq S_{i}$. La restriction à $U^{\prime}$ de $g \circ f$ est alors régulière.

Corollaire 4.15. Soient $m, n$ des entiers naturels et $k$ un entier surnaturel. Une application $k$-régulue de $\mathbf{R}^{n}$ dans $\mathbf{R}^{m}$ est continue pour la topologie $k$ régulue.

Démonstration. Soit $f: \mathbf{R}^{n} \rightarrow \mathbf{R}^{m}$ une application $k$-régulue et $F$ un fermé $k$ régulu de $\mathbf{R}^{m}$. Il existe une fonction $k$-régulue $g$ sur $\mathbf{R}^{m}$ dont l'ensemble des zéros est égal à $F$. D'après le Corollaire $4.14, g \circ f$ est $k$-régulue. L'image réciproque

$$
f^{-1}(F)=\mathcal{Z}(g \circ f)
$$

est donc un fermé $k$-régulu de $\mathbf{R}^{n}$.

Grâce au Corollaire 4.14, on est en mesure de montrer que l'anneau $\mathcal{R}^{k}\left(\mathbf{R}^{n}\right)$ n'est pas noethérien, lorsque $k$ est fini, même si la topologie $k$-régulue est noethérienne :

Proposition 4.16. Soient $n$ et $k$ des entiers naturels. L'anneau $\mathcal{R}^{k}\left(\mathbf{R}^{n}\right)$ n'est pas noethérien lorsque $n \geq 2$.

Démonstration. Pour $m \in \mathbf{N}$, soit $f_{m}$ la fonction $k$-régulue sur $\mathbf{R}^{n}$ définie par

$$
f_{m}=\frac{x_{2}^{3+k}}{x_{2}^{2}+\left(x_{1}-m\right)^{2}} .
$$

Soit $I_{m}$ l'idéal de $\mathcal{R}^{k}\left(\mathbf{R}^{n}\right)$ engendré par les fonctions $f_{0}, \ldots, f_{m}$. On montre comme dans [24, Ex. 6.11] que la suite d'idéaux croissante $I_{0}, I_{1}, \ldots$ n'est 
pas stationnaire. En fait, on montre par l'absurde que $I_{m+1} \neq I_{m}$. En effet, supposons que $f_{m+1}$ appartient à $I_{m}$. On peut donc écrire

$$
f_{m+1}=\sum_{i=1}^{m} g_{i} f_{i}
$$

où $g_{1}, \ldots, g_{m}$ sont des fonctions $k$-régulues sur $\mathbf{R}^{n}$. En restreignant à la courbe réelle algébrique $C=\{m+1\} \times \mathbf{R} \times\{0\}^{n-2}$, qu'on identifie avec $\mathbf{R}$, on obtient

$$
x_{2}^{1+k}=\sum_{i=1}^{m}\left(g_{i}\right)_{\mid C}\left(f_{i}\right)_{\mid C}
$$

sur $C$. D'après Corollaire 4.14 , les fonctions $\left(g_{i}\right)_{\mid C}$ et $\left(f_{i}\right)_{\mid C}$ sont $k$-régulues et donc régulières. De plus, la fonction

$$
\left(f_{i}\right)_{\mid C}=\frac{x_{2}^{3+k}}{x_{2}^{2}+(m+1-i)^{2}}
$$

s'annule au point $x_{2}=0$ avec multiplicité $3+k$, pour $i=1, \ldots, m$. Il s'ensuit que $x_{2}^{1+k}$ s'annule en $x_{2}=0$ avec multiplicité au moins $3+k$. Contradiction.

Jets de fonctions $k$-régulues. Soient $k, \ell$ et $n$ des entiers naturels, avec $\ell \leq k$. Soit $f$ une fonction de classe $\mathcal{C}^{k}$ sur un ouvert $U$ de $\mathbf{R}^{n}$. Habituellement, on écrit le $\ell$-ième polynôme de Taylor de $f$ en un point $a$ sous la forme

$$
\sum_{|I| \leq \ell} \frac{\partial^{|I|} f}{\partial x^{I}}(a) \frac{(x-a)^{I}}{I !} .
$$

Lorsqu'on s'intéresse à celui-ci comme fonction de $a$, on est amené à le noter

$$
\sum_{|I| \leq k} \frac{\partial^{|I|} f}{\partial x^{I}}(x) \frac{(y-x)^{I}}{I !}
$$

où $y$ désigne un système de coordonnées affine arbitraire sur $\mathbf{R}^{n}$. On appelle ce dernier le $\ell$-jet de $f$, et on le note $j_{\ell}(f)$.

Le fibré vectoriel trivial $J_{\ell}$ sur $\mathbf{R}^{n}$ de base

$$
\frac{(y-x)^{I}}{I !}, \quad|I| \leq \ell
$$

est le fibré des $\ell$-jets sur $\mathbf{R}^{n}$. Le $\ell$-jet $j_{\ell}(f)$ de $f$ est une section de $J_{\ell}$ de classe $\mathcal{C}^{k-\ell}$ au-dessus $U$.

Le jonglage notationnel ci-dessus a l'avantage que le 0 -jet $j_{0}(f)$ de $f$ coïncide avec $f$. Plus généralement, le coefficient de $(y-x)^{I} / I$ ! du $\ell$-jet de $f$ est égal à la dérivée partielle d'ordre $I$ de $f$.

Le fibré vectoriel des $\ell$-jets $J_{\ell}$ possède une structure de fibré en algèbres si on définit

$$
\frac{(y-x)^{I}}{I !} \cdot \frac{(y-x)^{I}}{J !}= \begin{cases}\left(\begin{array}{c}
I+J \\
I
\end{array}\right) \frac{(y-x)^{I+J}}{(I+J) !} & \text { si }|I+J| \leq \ell, \text { et } \\
0 & \text { sinon. }\end{cases}
$$

Si $f$ et $g$ sont des fonctions de classe $\mathcal{C}^{k}$ sur un ouvert $U$ de $\mathbf{R}^{n}$, on a

$$
j_{\ell}(f g)=j_{\ell}(f) j_{\ell}(g)
$$


sur $U$.

Comme le fibré vectoriel $J_{\ell}$ est libre d'une base explicitée, on peut identifier ses sections au-dessus d'un ouvert $U$ de $\mathbf{R}^{n}$ avec des applications de $U$ dans $\mathbf{R}^{N}$, où $N=N_{n, k, \ell}$ est le rang de $J_{\ell}$. Du coup, cela a un sens de parler de section rationnelle ou encore de section $m$-régulue de $J_{\ell}$, où $m$ est un entier surnaturel quelconque.

Lemme 4.17. Soit $\ell$ un entier naturel. Soit $f$ une fonction rationnelle sur $\mathbf{R}^{n}$, et soit $U$ son domaine. Le $\ell$-jet $j_{\ell}(f)$ est une section rationnelle de $J_{\ell}$ de domaine $U$.

Démonstration. Ecrivons $f=p / q$ sur $U$, où $p$ et $q$ sont des fonctions polynomiales, $q$ ne s'annulant pas sur $U$. On sait que la dérivée partielle d'ordre $I$ de $f$ est de la forme $r / q^{|I|+1}$, où $r$ est une fonction polynomiale sur $\mathbf{R}^{n}$. Il s'ensuit que $j_{\ell}(f)$ est une section rationnelle de $J_{\ell}$ de domaine $V \supseteq U$. Comme le coefficient de $(y-x)^{0} / 0$ ! est $p / q=f$, le domaine $V$ est égal à $U$.

Proposition 4.18. Soit $f$ une fonction réelle sur $\mathbf{R}^{n}$. La fonction $f$ est 1 régulue si et seulement si $f$ est rationnelle et la section rationnelle $j_{1}(f)$ de $J_{1}$ au-dessus de $\operatorname{dom}(f)$ s'étend de manière continue à $\mathbf{R}^{n}$.

Démonstration. L'implication directe est évidente compte tenu du Lemme 4.17. Montrons donc l'implication réciproque, et soit $f$ une fonction rationnelle telle que $j_{1}(f)$ s'étend de manière continue à $\mathbf{R}^{n}$. Notons $U$ le domaine de $f$. D'après l'hypothèse, $f$ est de classe $\mathcal{C}^{1}$ sur $U$. Soit $Z$ le complémentaire de $U$, i.e., $Z=\operatorname{indet}(f)$. Comme $Z$ est un sous-ensemble Zariski fermé strictement contenu dans $\mathbf{R}^{n}$, il existe un système de coordonnées affine $x^{\prime}$ sur $\mathbf{R}^{n}$ avec la propriété suivante. Aucune des fibres des projections associées

$$
p_{i}: \mathbf{R}^{n} \longrightarrow \mathbf{R}^{n-1}
$$

omettant la $i$-ième coordonnée $x_{i}^{\prime}$ n'est contenue entièrement dans $Z$. Quitte à remplacer $x$ par $x^{\prime}$, on peut supposer que la propriété est satisfaite par le système des coordonnées $x$, i.e., pour tout $i=1, \ldots, n$ et pour tout $a \in \mathbf{R}^{n-1}$, l'intersection $Z \cap p_{i}^{-1}(a)$ est un ensemble fini.

Comme $f$ est de classe $\mathcal{C}^{1}$ sur $U$, les dérivées partielles $f_{i}=\partial f / \partial x_{i}$, $i=1, \ldots, n$, de $f$ existent et sont continues sur $U$. De plus, d'après l'hypothèse, elles s'étendent par continuité sur $\mathbf{R}^{n}$ tout entier. En particulier, $f_{i}$ s'étend par continuité sur $p_{i}^{-1}(a)$ pour tout $a \in \mathbf{R}^{n-1}$ et pour tout $i$. D'après un résultat classique en analyse [31, Theorem 11.7], cela implique que la restriction de $f_{i}$ à $p_{i}^{-1}(a)$ est de classe $\mathcal{C}^{1}$ sur $p_{i}^{-1}(a)$, pour tout $a$ et $i$. (Nous utilisons ici la finitude de $Z \cap p_{i}^{-1}(a)$.) Autrement dit, $f$ est partiellement différentiable sur $\mathbf{R}^{n}$ tout entier, et ses dérivées partielles d'ordre 1 sont continues sur $\mathbf{R}^{n}$. D'après un autre résultat classique d'analyse [29, Thm 9.21], $f$ est alors de classe $\mathcal{C}^{1}$ $\operatorname{sur} \mathbf{R}^{n}$.

Théorème 4.19. Soit $k$ un entier naturel. Soit $f$ une fonction réelle sur $\mathbf{R}^{n}$. La fonction $f$ est $k$-régulue si et seulement si $f$ est rationnelle et la section rationnelle $j_{k}(f)$ de $J_{k}$ au-dessus de $\operatorname{dom}(f)$ s'étend de manière continue à $\mathbf{R}^{n}$.

Démonstration. L'implication directe étant encore évidente, on démontre l'implication réciproque. Le cas $k=0$ est trivial. Supposons que $k>0$ et soit $f$ 
une fonction rationnelle de domaine $U$ telle que la section $j_{k}(f)$ au-dessus de $U$ s'étend de manière continue à $\mathbf{R}^{n}$. Les dérivées partielles $f_{I}$ d'ordre $k-1$ de $f$ ont des 1-jets qui s'étendent de manière continue à $\mathbf{R}^{n}$. D'après la Proposition 4.18, ces dérivées partielles sont toutes 1-régulues. Il s'ensuit que $f$ est $k$-régulue.

Théorème 4.20. Soient $k$ un entier surnaturel et $\ell$ un entier naturel $\leq k$. Soit $f$ une fonction réelle sur $\mathbf{R}^{n}$. La fonction $f$ est $k$-régulue si et seulement si $f$ est rationnelle et la section rationnelle $j_{\ell}(f)$ de $J_{\ell}$ au-dessus de $\operatorname{dom}(f)$ s'étend de manière $\mathcal{C}^{k-\ell} \grave{a} \mathbf{R}^{n}$.

Démonstration. On peut supposer que $k$ est fini. Comme précédemment, il suffit de démontrer l'implication indirecte. Soit $f$ une fonction rationnelle dont toutes les dérivées d'ordre $\leq \ell$ sont $(k-\ell)$-régulues. D'après l'implication directe du Théorème 4.19 , le $k$-jet de $f$ s'étend de manière continue à $\mathbf{R}^{n}$. D'après l'implication indirecte du même théorème, $f$ est $k$-régulue.

\section{Nullstellenzatz et Théorèmes A et B}

L'inégalité de Łojasiewicz. Un rôle clé dans la suite est joué par une version $k$-régulue d'un corollaire de l'inégalité de Łojasiewicz, cf. [6, Prop. 2.6.4].

Lemme 5.1. Soient $n$ et $k$ des entiers naturels. Soit $f$ une fonction $k$-régulue sur $\mathbf{R}^{n}$. Si

$$
g: \mathcal{D}(f) \rightarrow \mathbf{R}
$$

est $k$-régulue, alors il existe un entier naturel $N$ tel que l'extension à $\mathbf{R}^{n}$ par 0 de $f^{N} g$ est $k$-régulue.

Démonstration. Si $k=0$, le résultat est classique, cf. [6, Prop. 2.6.4]. Par conséquent, pour tout entier naturel $k$, il existe un entier naturel $M$ tel que pour tout $I$ avec $|I| \leq k$, l'extension de

$$
f^{M} \cdot \frac{\partial^{|I|} g}{\partial x^{I}}
$$

par 0 sur $\mathbf{R}^{n}$ est continue. Soit $N=M+k$. La fonction $f^{N} g$ étendue par 0 sur $\mathbf{R}^{n}$ est de classe $\mathcal{C}^{k}$. En effet, toutes les dérivées partielles d'ordre inférieur ou égal à $k$ se prolongent par continuité par 0 sur $\mathbf{R}^{n}$. La preuve pour tout entier naturel $k$ se déduit donc du cas $k=0$.

On peut préciser cela en termes de $k$-jets. Comme $f$ est $k$-régulue sur $\mathbf{R}^{n}$, tout coefficient du $k$-jet $j_{k}\left(f^{N}\right)$ de $f^{N}$ est de la forme $f^{M} \cdot h$, où $h$ est une fonction régulue sur $\mathbf{R}^{n}$. Du coup, $j_{k}\left(f^{N}\right)$ lui-même est de la forme $f^{M} \cdot s$, où $s$ est une section régulue de $J_{k}$ au dessus de $\mathbf{R}^{n}$. Il s'ensuit que l'extension par 0 de

$$
j_{k}\left(f^{N} g\right)=j_{k}\left(f^{N}\right) j_{k}(g)=f^{M} j_{k}(g)
$$

est une section régulue de $J_{k}$ au-dessus $\mathbf{R}^{n}$ tout entier. D'après le Théorème 4.19 , la fonction $f^{N} g$ est $k$-régulue sur $\mathbf{R}^{n}$.

Le lemme ci-dessus nous sera utile sous la forme suivante : 
Lemme 5.2. Soient $n$ et $k$ des entiers naturels. Soit $U$ un ouvert $k$-régulu de $\mathbf{R}^{n}$, et soit $f$ une fonction $k$-régulue sur $U$. Si

$$
g: \mathcal{D}(f) \rightarrow \mathbf{R}
$$

est une fonction $k$-régulue, il existe un entier naturel $N$ tel que l'extension à $U$ par 0 de $f^{N} g$ est $k$-régulue.

Démonstration. Soit $h$ une fonction $k$-régulue sur $\mathbf{R}^{n}$ telle que $\mathcal{D}(h)=U$. D'après le Lemme 5.1, il existe un entier naturel $M$ tel que l'extension par 0 de $h^{M} f$ est $k$-régulue sur $\mathbf{R}^{n}$. Comme $g$ est $k$-régulue sur $\mathcal{D}\left(h^{M} f\right)$, il existe encore d'après le Lemme 5.1, un entier naturel $N$ tel que l'extension par 0 de $\left(h^{M} f\right)^{N} g$ est $k$-régulue sur $\mathbf{R}^{n}$. Il s'ensuit que l'extension par 0 de $f^{N} g$ à $U=\mathcal{D}(h)$ est $k$-régulue.

Remarquons que les deux énoncés ci-dessus sont faux lorsque $k=\infty$. Un contre-exemple est le suivant. Soit $f$ la fonction sur $\mathbf{R}^{2}$ définie par $f(x)=$ $x^{2}+y^{2}$, et soit $g$ la fonction sur $\mathbf{R}^{2} \backslash\{0\}$ définie par $g(x, y)=1 /\left(x^{2}+2 y^{2}\right)$. Les fonctions $f$ et $g$ sont bien de classe $\mathcal{C}^{\infty}$, mais l'extension à $\mathbf{R}^{2}$ par 0 de $f^{N} g$ n'est de classe $\mathcal{C}^{\infty}$ pour aucun entier naturel $N$.

Le faisceau des fonctions $k$-régulues sur $\mathbf{R}^{n}$. Soient $n$ un entier naturel et $k$ un entier surnaturel. Soit $U$ un ouvert $k$-régulu de $\mathbf{R}^{n}$. La topologie de Zariski sur $U$ est la topologie induite par la topologie de Zariski sur $\mathbf{R}^{n}$. Une fonction réelle $f$ définie sur $U$ est $k$-régulue sur $U$ si $f$ est de classe $\mathcal{C}^{k}$ sur $U$, et il existe des fonctions polynomiales $p$ et $q$ sur $\mathbf{R}^{n}$ telles que $f=p / q$ sur un ouvert de Zariski dense de $U$. On note $\mathcal{R}^{k}(U)$ l'ensemble des fonctions $k$-régulues sur $U$. C'est un anneau sous les opérations habituelles. Si $V$ est un ouvert $k$-régulu de $\mathbf{R}^{n}$ contenu dans $U$, l'application de restriction de $\mathcal{R}^{k}(U)$ dans $\mathcal{R}^{k}(V)$ est un morphisme d'anneaux. Il est clair que $\mathcal{R}^{k}$ est donc un préfaisceau.

Proposition 5.3. Soient $n$ un entier naturel et $k$ un entier surnaturel. Le préfaisceau $\mathcal{R}^{k}$ est un faisceau sur $\mathbf{R}^{n}$ pour la topologie $k$-régulue.

Démonstration. Il suffit de montrer l'énoncé suivant. Soit $\left\{U_{i}\right\}_{i \in I}$ un recouvrement ouvert d'un ouvert $k$-régulu $U$ de $\mathbf{R}^{n}$. Supposons que $f_{i}$ est une fonction $k$-régulue sur $U_{i}$, pour tout $i$, telles que

$$
\left(f_{i}\right)_{\mid U_{i} \cap U_{j}}=\left(f_{j}\right)_{\mid U_{i} \cap U_{j}}
$$

pour tout $i, j$. Alors il existe une fonction $k$-régulue $f$ sur $U$ dont la restriction à $U_{i}$ est égale à $f_{i}$. En effet, il existe une fonction $f$ sur $U$ de classe $\mathcal{C}^{k}$ dont la restriction à $U_{i}$ est égale à $f_{i}$ pour tout $i$. Pour montrer que $f$ est $k$-régulue, on peut supposer que $U$ est non vide. Du coup, il existe $i$ tel que $U_{i}$ est non vide. Comme $\mathbf{R}^{n}$ est irréductible pour la topologie $k$-régulue, tout ouvert $k$-régulu non vide de $\mathbf{R}^{n}$ est dense. En particulier, un ouvert de Zariski dense dans $U_{i}$ est aussi dense dans $U$. Comme $f_{i}$ est régulière sur un ouvert de Zariski dense dans $U_{i}$, la fonction $f$ est régulière sur un ouvert de Zariski dense dans $U$.

Corollaire 5.4. Soient $n$ un entier naturel et $k$ un entier surnaturel. Le faisceau $\mathcal{R}^{k}$ sur $\mathbf{R}^{n}$ est un faisceau en $\mathbf{R}$-algèbres locales. La paire $\left(\mathbf{R}^{n}, \mathcal{R}^{k}\right)$ est un espace localement annelé en $\mathbf{R}$-algèbres. 
Corollaire 5.5. Soient $m, n$ des entiers naturels et $k$ un entier surnaturel. Une application $k$-régulue de $\mathbf{R}^{n}$ dans $\mathbf{R}^{m}$ induit naturellement un morphisme d'espaces localement annelés en $\mathbf{R}$-algèbres de $\left(\mathbf{R}^{n}, \mathcal{R}^{k}\right)$ dans $\left(\mathbf{R}^{m}, \mathcal{R}^{k}\right)$. Cela établit une bijection entre l'ensemble des applications $k$-régulues de $\mathbf{R}^{n}$ dans $\mathbf{R}^{m}$ et l'ensemble des morphismes d'espaces localement annelés en $\mathbf{R}$ algèbres de $\left(\mathbf{R}^{n}, \mathcal{R}^{k}\right)$ dans $\left(\mathbf{R}^{m}, \mathcal{R}^{k}\right)$.

Démonstration. Soit $f$ une application $k$-régulue de $\mathbf{R}^{n}$ dans $\mathbf{R}^{m}$. D'après le Corollaire 4.15, $f$ est une application continue de $\mathbf{R}^{n}$ dans $\mathbf{R}^{m}$, par rapport aux topologies $k$-régulues. De plus, si $g$ est une fonction $k$-régulue sur un ouvert $k$-régulu $U$ de $\mathbf{R}^{m}$, la composition $f^{\#}(g)=g \circ f$ est une fonction $k$-régulue sur l'ouvert $k$-régulu $f^{-1}(U)$ de $\mathbf{R}^{n}$, d'après le Corollaire 4.14. On en déduit donc un morphisme d'espaces localement annelés en $\mathbf{R}$-algèbres

$$
\left(f, f^{\#}\right):\left(\mathbf{R}^{n}, \mathcal{R}^{k}\right) \longrightarrow\left(\mathbf{R}^{m}, \mathcal{R}^{k}\right) .
$$

Réciproquement, étant donné un tel morphisme $\left(f, f^{\#}\right)$, l'application $f$ est une application $k$-régulue de $\mathbf{R}^{n}$ dans $\mathbf{R}^{m}$ car la $i$-ième fonction coordonnée $f_{i}$ de $f$ est $f^{\#}\left(x_{i}\right)$ et est une fonction $k$-régulue sur $\mathbf{R}^{n}$, pour $i=1, \ldots, m$.

Grâce à la version $k$-régulue de l'inégalité de Łojasiewicz, on a une description de l'anneau des sections du faisceau $\mathcal{R}^{k}$ au-dessus d'un ouvert comme une localisation de $\mathcal{R}^{k}\left(\mathbf{R}^{n}\right)$, lorsque $k$ est fini :

Proposition 5.6. Soient $n$ et $k$ des entiers naturels. Soit $U$ un ouvert $k$-régulu de $\mathbf{R}^{n}$, et soit $f$ une fonction k-régulue sur $\mathbf{R}^{n}$ telle que $\mathcal{D}(f)=U$. Alors, le morphisme de restriction de $\mathcal{R}^{k}\left(\mathbf{R}^{n}\right)$ dans $\mathcal{R}^{k}(U)$ induit un isomorphisme

$$
\mathcal{R}^{k}\left(\mathbf{R}^{n}\right)_{f} \cong \mathcal{R}^{k}(U) .
$$

Démonstration. Soit $\varphi: \mathcal{R}^{k}\left(\mathbf{R}^{n}\right) \rightarrow \mathcal{R}^{k}(U)$ le morphisme de restriction. Comme la restriction de $f$ à $U$ ne s'annule pas, $\varphi$ induit un morphisme

$$
\varphi_{f}: \mathcal{R}^{k}\left(\mathbf{R}^{n}\right)_{f} \longrightarrow \mathcal{R}^{k}(U) .
$$

On montre que $\varphi_{f}$ est un isomorphisme.

Soit $g \in \mathcal{R}^{k}(U)$. D'après le Lemme 5.2, il existe un entier naturel $N$ tel que $f^{N} g$ se prolonge en une fonction $k$-régulue sur $\mathbf{R}^{n}$. Par conséquent $\varphi_{f}$ est surjectif.

Pour montrer que $\varphi_{f}$ est injectif, il suffit de montrer l'énoncé suivant. Pour toute fonction $k$-régulue $h$ sur $\mathbf{R}^{n}$ dont la restriction à $U$ est identiquement nulle, la fonction $f h$ est identiquement 0 sur $\mathbf{R}^{n}$. Cet énoncé est clair lorsque $U=\emptyset$, car $f=0$ dans ce cas. Si $U \neq \emptyset$, alors $U$ est dense dans $\mathbf{R}^{n}$ pour la topologie euclidienne. Il s'ensuit que $h=0$ sur $\mathbf{R}^{n}$, et en particulier, $f h=0$.

Idéaux radicaux de fonctions régulues. On rappelle qu'un idéal $I$ d'un anneau commutatif $A$ est réel s'il satisfait la propriété suivante : Si $f_{1}^{2}+\ldots+$ $f_{m}^{2} \in I$, avec $f_{1}, \ldots, f_{m} \in A$, alors $f_{i} \in I$ pour chaque $i=1, \ldots, m$.

Proposition 5.7. Soient $n$ et $k$ des entiers naturels. Soit $I \subseteq \mathcal{R}^{k}\left(\mathbf{R}^{n}\right)$ un idéal radical. Alors I est un idéal réel. 
Démonstration. On suppose $f_{1}^{2}+\ldots+f_{m}^{2} \in I$ avec $f_{1}, \ldots, f_{m} \in \mathcal{R}^{k}\left(\mathbf{R}^{n}\right)$. Pour $i=1, \ldots, k$ on a $\frac{f_{i}^{3+k}}{\sum_{i=1}^{m} f_{i}^{2}} \in \mathcal{R}^{k}\left(\mathbf{R}^{n}\right)$. En effet c'est la composée des applications $k$-régulues $\mathbf{R}^{n} \rightarrow \mathbf{R}^{m}, x \mapsto\left(f_{1}(x), \ldots, f_{m}(x)\right)$ et de $\mathbf{R}^{m} \rightarrow$ $\mathbf{R},\left(x_{1}, \ldots, x_{m}\right) \mapsto \frac{x_{i}^{3+k}}{\sum_{i=1}^{m} x_{i}^{2}}$, qui est bien $k$-régulue d'après le Corollaire 4.14. Donc $f_{i}^{3+k}=\frac{f_{i}^{3+k}}{\sum_{i=1}^{m} f_{i}^{2}}\left(\sum_{i=1}^{m} f_{i}^{2}\right) \in I$. L'idéal $I$ étant radical on obtient $f_{i} \in I$.

Remarque 5.8. La proposition précédente montre que l'idéal engendré par $x^{2}+y^{2}$ dans $\mathcal{R}^{k}\left(\mathbf{R}^{2}\right)$ n'est pas radical. En effet, s'il est radical alors il est réel et par conséquent $x \in \mathcal{R}^{k}\left(\mathbf{R}^{2}\right) \cdot\left(x^{2}+y^{2}\right)$ et donc $x=f .\left(x^{2}+y^{2}\right)$ avec $f \in \mathcal{R}^{k}\left(\mathbf{R}^{2}\right)$. Mais $f=\frac{x}{x^{2}+y^{2}}$ n'est clairement pas continue en l'origine.

Soit $I$ un idéal radical dans $\mathcal{R}^{k}\left(\mathbf{R}^{n}\right)$. On sait que $I$ est un idéal réel d'après la Proposition 5.7. On montre que cette propriété est conservée par intersection avec les polynômes.

Lemme 5.9. Soit I un idéal radical dans $\mathcal{R}^{k}\left(\mathbf{R}^{n}\right)$. Alors $J=I \cap \mathbf{R}\left[x_{1}, \ldots, x_{n}\right]$ est un idéal réel et on a $\mathcal{I}(\mathcal{Z}(J))=J$.

Démonstration. L'idéal $J$ est réel car $I$ est réel (Proposition 5.7). On obtient $\mathcal{I}(\mathcal{Z}(J))=J$ par le Nullstellensatz réel [6, Thm. 4.1.4]

Soit $J$ un idéal de $\mathbf{R}\left[x_{1}, \ldots, x_{n}\right]$. On regarde le comportement de $J$ quand on l'étend dans les fonctions régulues.

On fixe d'abord quelques notations.

Notations et Définitions 5.10. Soit $x \in \mathbf{R}^{n}$. Dans la suite, on note

$$
\begin{gathered}
\mathfrak{m}_{x}=\left\{p \in \mathbf{R}\left[x_{1}, \ldots, x_{n}\right] \mid p(x)=0\right\} \\
\mathfrak{M}_{x}=\left\{f \in \mathcal{R}^{k}\left(\mathbf{R}^{n}\right) \mid f(x)=0\right\}
\end{gathered}
$$

les idéaux maximaux de $\mathbf{R}\left[x_{1}, \ldots, x_{n}\right]$ et $\mathcal{R}^{k}\left(\mathbf{R}^{n}\right)$ respectivement, des polynômes et des fonctions régulues qui s'annulent en $x$. Plus généralement, si $A \subseteq \mathbf{R}^{n}$, on note dans la suite

$$
\mathcal{I}(A)=\left\{p \in \mathbf{R}\left[x_{1}, \ldots, x_{n}\right] \mid p(x)=0 \forall x \in A\right\}
$$

et

$$
\mathcal{I}_{\mathcal{R}^{k}}(A)=\left\{f \in \mathcal{R}^{k}\left(\mathbf{R}^{n}\right) \mid f(x)=0 \forall x \in A\right\} .
$$

Examinons l'exemple suivant. Soit $(x, y)=\mathfrak{m}_{O}=\{p \in \mathbf{R}[x, y] \mid p(O)=0\}$ l'idéal maximal de $\mathbf{R}[x, y]$ où $O$ est l'origine de $\mathbf{R}^{2}$. On montre que $\mathcal{R}^{0}\left(\mathbf{R}^{2}\right) \cdot \mathfrak{m}_{O}$ n'est pas maximal dans $\mathcal{R}^{0}\left(\mathbf{R}^{2}\right)$.

On a clairement $\mathcal{R}^{0}\left(\mathbf{R}^{2}\right) \cdot \mathfrak{m}_{O} \subseteq \mathfrak{M}_{O}$. On a aussi $f=\frac{x y^{2}}{x^{2}+y^{2}} \in \mathfrak{M}_{O}$. On va montrer par contre que $f \notin \mathcal{R}^{0}\left(\mathbf{R}^{2}\right) \cdot \mathfrak{m}_{O}$. Supposons que $f$ s'écrive $f=x . g+y . h$ avec $g, h \in \mathcal{R}^{0}\left(\mathbf{R}^{2}\right)$. Alors on peut écrire

$f=g(O) \cdot x+h(O) \cdot y+(g-g(O)) \cdot x+(h-h(O)) \cdot y=a \cdot x+b \cdot y+o\left(\sqrt{x^{2}+y^{2}}\right)$.

Mais alors $f$ serait différentiable à l'origine et on obtient une contradiction. Cet exemple montre que l'on peut s'attendre à quelques surprises. 
Théorème 5.11. Soit $J$ un idéal réel de $\mathbf{R}\left[x_{1}, \ldots, x_{n}\right]$. Alors

$$
\operatorname{Rad}\left(\mathcal{R}^{k}\left(\mathbf{R}^{n}\right) . J\right)=\mathcal{I}_{\mathcal{R}^{k}}(\mathcal{Z}(J)) .
$$

Démonstration. On note $I=\mathcal{R}^{k}\left(\mathbf{R}^{n}\right) . J$. Soit $f \in \operatorname{Rad}(I)$, il existe $r \in \mathbf{N}^{*}$ tel que $f^{r} \in I$. Comme $J=\mathcal{I}(\mathcal{Z}(J))\left[6\right.$, Thm. 4.1.4] dans $\mathbf{R}\left[x_{1}, \ldots, x_{n}\right]$ car $J$ est réel, on en déduit que $f^{r}$ s'annule identiquement sur $\mathcal{Z}(J)$. Par conséquent $f$ s'annule aussi identiquement sur $\mathcal{Z}(J)$ i.e. $f \in \mathcal{I}_{\mathcal{R}^{k}}(\mathcal{Z}(J))$. On a montré une inclusion.

Pour l'inclusion réciproque, on suppose que $J$ est l'idéal $J=\left(p_{1}, \ldots, p_{l}\right)$ avec $p_{i} \in \mathbf{R}\left[x_{1}, \ldots, x_{n}\right]$. Alors on a $V=\mathcal{Z}(J)=\mathcal{Z}(s)$ avec $s=p_{1}^{2}+\ldots+p_{l}^{2}$. Soit $f \in \mathcal{R}^{k}\left(\mathbf{R}^{n}\right)$ tel que $f \in \mathcal{I}_{\mathcal{R}^{k}\left(\mathbf{R}^{n}\right)}(V)$. Soit $g=\frac{1}{s}$, c'est une fonction $k$-régulue sur $\mathbf{R}^{n} \backslash V$ donc sur $\mathcal{D}(f)$. Par le Lemme 5.1, il existe un entier positif $N$ tel que $h=f^{N} . g$ étendue par 0 sur $\mathcal{Z}(f)$ est $k$-régulue sur $\mathbf{R}^{n}$. On a clairement $h \in \mathcal{I}_{\mathcal{R}^{k}}(\mathcal{Z}(f)) \subseteq \mathcal{I}_{\mathcal{R}^{k}}(V)$ et de plus $f^{N}=$ h.s : en effet, sur $\mathcal{D}(f)$ c'est évident et si $x \in Z(f)$ on a aussi $f^{N}(x)=h(x) s(x)=0$ (on utilisera plusieurs fois dans la suite ce même argument). Par conséquent

$$
f^{N}=h . s \in \mathcal{R}^{k}\left(\mathbf{R}^{n}\right) . J=I
$$

car $s \in J$.

Dans le cas où $J$ est maximal, on obtient

Corollaire 5.12. On a

$$
\operatorname{Rad}\left(\mathcal{R}^{k}\left(\mathbf{R}^{n}\right) \cdot \mathfrak{m}_{x}\right)=\mathfrak{M}_{x}
$$

Corollaire 5.13. On suppose $k=0$ et soit $f \in \mathfrak{M}_{x}$. Il existe un entier strictement positif $N$ tel que $f^{N}$ soit différentiable en $x$ avec $D(f)_{x}=0$.

Démonstration. On suppose $x=O$. En regardant la preuve du théorème précédent, il existe un entier strictement positif $N$ tel que $f^{N}=\left(x_{1}^{2}+\ldots+x_{n}^{2}\right) . h$ avec $h \in \mathcal{R}^{0}\left(\mathbf{R}^{n}\right)$. On a donc $f^{N}=x_{1} \cdot h_{1}+\ldots+x_{n} . h_{n}$ avec $h_{i} \in \mathcal{R}^{0}\left(\mathbf{R}^{n}\right)$ vérifiant $h_{i}(O)=0$. On peut donc écrire $f^{N}=o\left(\sqrt{x_{1}^{2}+\ldots+x_{n}^{2}}\right)$ ce qui termine la preuve.

L'anneau $\mathcal{R}^{k}(\mathbf{R})$ est radicalement principal. Soit $A$ un anneau. On dira qu'un idéal $I$ de $A$ est radicalement principal s'il existe $f \in I$ tel que

$$
\operatorname{Rad}(I)=\operatorname{Rad}(f) .
$$

Dans ce cas, on dira aussi que $f$ est un générateur radical de $I$. On dit que $A$ est radicalement principal si tous ses idéaux le sont.

Proposition 5.14. Soient $n$ et $k$ des entiers naturels. Soit $I$ un idéal de $\mathcal{R}^{k}\left(\mathbf{R}^{n}\right)$, et supposons que $f \in I$ soit telle que $\mathcal{Z}(f)=\mathcal{Z}(I)$. Alors, $f$ est un générateur radical de $I$, i.e.,

$$
\operatorname{Rad}(f)=\operatorname{Rad}(I) .
$$

Démonstration. Comme $f \in I$, on a bien-sûr l'inclusion $\operatorname{Rad}(f) \subseteq \operatorname{Rad}(I)$. Pour montrer l'inclusion réciproque, il suffit de montrer que $\operatorname{Rad}(f) \supseteq I$. Soit $g \in I$. La fonction $1 / f$ est bien définie et $k$-régulue sur $\mathcal{D}(g)$. D'après le Lemme 5.1, il existe un entier naturel $N$ tel que l'extension $h$ par 0 
de $g^{N} / f$ sur $\mathcal{Z}(g)$ est encore $k$-régulue. On a $g^{N}=f h$ sur $\mathbf{R}^{n}$. Cela implique que $g \in \operatorname{Rad}(f)$.

Proposition 5.15. Soient $n$ et $k$ des entiers naturels. L'anneau $\mathcal{R}^{k}\left(\mathbf{R}^{n}\right)$ est radicalement principal. Plus précisément, soit I un idéal de $\mathcal{R}^{k}\left(\mathbf{R}^{n}\right)$. Alors il existe une fonction $k$-régulue $f \in I$ telle que

$$
\operatorname{Rad}(f)=\operatorname{Rad}(I) .
$$

Démonstration. D'après le Théorème 4.3 , la topologie $k$-régulue sur $\mathbf{R}^{n}$ est noethérienne. Il existe donc un nombre fini de fonctions $k$-régulues $f_{1}, \ldots, f_{m}$ dans $I$ telles que

Soit

$$
\mathcal{Z}\left(f_{1}, \ldots, f_{m}\right)=\mathcal{Z}(I)
$$

$$
f=f_{1}^{2}+\cdots+f_{m}^{2}
$$

Comme $f \in I$ et $\mathcal{Z}(f)=\mathcal{Z}(I)$, la fonction $f$ est un générateur radical de $I$ d'après la Proposition 5.14.

Proposition 5.16. Soient $n$ et $k$ des entiers naturels. Soit $f$ une fonction $k$ régulue sur $\mathbf{R}^{n}$, et soit I un idéal de $\mathcal{R}^{k}\left(\mathbf{R}^{n}\right)$. Alors, $f \in \operatorname{Rad}(I)$ si et seulement si $\mathcal{Z}(f) \supseteq \mathcal{Z}(I)$.

Démonstration. D'après la Proposition 5.15, il existe $g \in I$ telle que $\operatorname{Rad}(g)=$ $\operatorname{Rad}(I)$. En particulier, on a

$$
\mathcal{Z}(g)=\mathcal{Z}(\operatorname{Rad}(g))=\mathcal{Z}(\operatorname{Rad}(I))=\mathcal{Z}(I) .
$$

L'énoncé est donc une conséquence de la proposition suivante.

Proposition 5.17. Soient $n$ et $k$ des entiers naturels. Soient $f$ et $g$ des fonctions $k$-régulues sur $\mathbf{R}^{n}$. Alors, $f \in \operatorname{Rad}(g)$ si et seulement si $\mathcal{Z}(f) \supseteq \mathcal{Z}(g)$.

Démonstration. Supposons que $f \in \operatorname{Rad}(g)$, et soit $x \in \mathcal{Z}(g)$. Il existe un entier naturel $N$ tel que $f^{N} \in(g)$, i.e., $f^{N}=g h$ pour une certaine fonction $k$-régulue $h$ sur $\mathbf{R}^{n}$. Comme $g(x)=0$, on a $f^{N}(x)=0$ et donc $f(x)=0$, i.e., $x \in \mathcal{Z}(f)$.

Réciproquement, supposons que $\mathcal{Z}(f) \supseteq \mathcal{Z}(g)$. Cela veut dire que $g$ ne s'annule pas sur $\mathcal{D}(f)$. En particulier, la fonction $1 / g$ existe sur $\mathcal{D}(f)$ et y est $k$-régulue. D'après la version $k$-régulue de l'inégalité de Łojasiewicz, il existe un entier naturel $N$ tel que l'extension par 0 de $f^{N} / g$ sur $\mathcal{Z}(f)$ est $k$-régulue sur $\mathbf{R}^{n}$. Notons cette extension par $h$. On a donc $f^{N}=g h$, i.e., $f \in \operatorname{Rad}(g)$.

Corollaire 5.18. Soient $n$ et $k$ des entiers naturels. Soient $f$ et $g$ des fonctions $k$-régulues sur $\mathbf{R}^{n}$. Alors, $\operatorname{Rad}(f)=\operatorname{Rad}(g)$ si et seulement si $\mathcal{Z}(f)=$ $\mathcal{Z}(g)$.

En utilisant Proposition 5.15 encore, on obtient la caractérisation géométrique suivante des générateurs radicaux d'un idéal de fonctions $k$-régulues :

Corollaire 5.19. Soient $n$ et $k$ des entiers naturels. Soit $f$ une fonction $k$ régulue sur $\mathbf{R}^{n}$, et soit I un idéal de $\mathcal{R}^{k}\left(\mathbf{R}^{n}\right)$. Alors, $\operatorname{Rad}(f)=\operatorname{Rad}(I)$ si et seulement si $\mathcal{Z}(f)=\mathcal{Z}(I)$.

Ou encore plus généralement : 
Corollaire 5.20. Soient $n$ et $k$ des entiers naturels. Soient $I$ et $J$ des idéaux de $\mathcal{R}^{k}\left(\mathbf{R}^{n}\right)$. Alors, $\operatorname{Rad}(I)=\operatorname{Rad}(J)$ si et seulement si $\mathcal{Z}(I)=\mathcal{Z}(J)$. En particulier, deux idéaux radicaux de $\mathcal{R}^{k}\left(\mathbf{R}^{n}\right)$ sont égaux si et seulement s'ils ont le même ensemble des zéros.

En résumé :

Théorème 5.21. Soient $n$ et $k$ des entiers naturels. L'anneau $\mathcal{R}^{k}\left(\mathbf{R}^{n}\right)$ est radicalement principal. Plus précisément, soit I un idéal de $\mathcal{R}^{k}\left(\mathbf{R}^{n}\right)$. Alors il existe une fonction $k$-régulue $f \in I$ telle que

$$
\operatorname{Rad}(f)=\operatorname{Rad}(I) .
$$

i.e. telle que

$$
\mathcal{Z}(f)=\mathcal{Z}(I)
$$

\section{Le Nullstellensatz $k$-régulu.}

Notation 5.22. Soient $n$ un entier naturel et $k$ un entier surnaturel. Si $V$ est un sous-ensemble de $\mathbf{R}^{n}$, on note $\mathcal{I}_{\mathcal{R}_{k}}(V)$, ou maintenant simplement $\mathcal{I}(V)$ s'il n'y a pas de confusion possible, l'ensemble des fonctions $k$-régulues sur $\mathbf{R}^{n}$ s'annulant sur $V$, i.e.,

$$
\mathcal{I}(V)=\left\{f \in \mathcal{R}^{k}\left(\mathbf{R}^{n}\right) \mid f(x)=0 \forall x \in V\right\} .
$$

Il est clair que $\mathcal{I}(V)$ est un idéal radical dans l'anneau $\mathcal{R}^{k}\left(\mathbf{R}^{n}\right)$.

Proposition 5.23 (Nullstellensatz faible). Soient $n$ un entier naturel et $k$ un entier surnaturel. Soit I un idéal de l'anneau $\mathcal{R}^{k}\left(\mathbf{R}^{n}\right)$ des fonctions $k$-régulues sur $\mathbf{R}^{n}$. Si $\mathcal{Z}(I)=\emptyset$ dans $\mathbf{R}^{n}$, alors $I=\mathcal{R}^{k}\left(\mathbf{R}^{n}\right)$.

Démonstration. Il existe $f \in I$ tel que $\mathcal{Z}(f)=\mathcal{Z}(I)$, en effet, si $k$ est un entier naturel c'est le Théorème 5.21, et si $k=\infty$, c'est la noethérianité de l'anneau des fonctions régulières. Supposons que $\mathcal{Z}(I)=\emptyset$. Cela veut dire que la fonction $k$-régulue $f$ n'a pas de zéros dans $\mathbf{R}^{n}$. Elle est donc inversible dans $\mathcal{R}^{k}\left(\mathbf{R}^{n}\right)$. Par conséquent $1=f \cdot \frac{1}{f} \in I$ i.e. $I=\mathcal{R}^{k}\left(\mathbf{R}^{n}\right)$.

Nous venons de voir que le Nullstellensatz faible est valable $\operatorname{sur} \mathcal{R}^{k}\left(\mathbf{R}^{n}\right)$ pour tout entier surnaturel $k$. Par contre, le Nullstellensatz fort $(\mathcal{I}(\mathcal{Z}(I))=\operatorname{Rad}(I)$ cf. e.g. [12, Theorem 5.1, p. 49]) n'est pas valable pour les fonctions $\infty$-régulues. Un contre-exemple est le suivant. Soit $I$ l'idéal de $\mathcal{R}^{\infty}\left(\mathbf{R}^{2}\right)$ engendré par $x^{2}+y^{2}$. L'ensemble des zéros $\mathcal{Z}(I)$ de $I$ est égal à l'origine de $\mathbf{R}^{2}$. La fonction $x$ s'annule bien sur $\mathcal{Z}(I)$, mais n'appartient pas à l'idéal radical $\operatorname{Rad}(I)$ de $I$ dans $\mathcal{R}^{\infty}\left(\mathbf{R}^{2}\right)$. En effet, l'extension à $\mathbf{R}^{2}$ de la fonction $x^{N} /\left(x^{2}+y^{2}\right)$ n'est de classe $\mathcal{C}^{\infty}$ pour aucun entier naturel $N$.

Théorème 5.24 (Nullstellensatz). Soient $n$ et $k$ des entiers naturels. Soit I un idéal (non-nécessairement de type fini) de $\mathcal{R}^{k}\left(\mathbf{R}^{n}\right)$. Alors,

$$
\mathcal{I}(\mathcal{Z}(I))=\operatorname{Rad}(I) \text {. }
$$

Démonstration. Soit $I$ un idéal quelconque de $\mathcal{R}^{k}\left(\mathbf{R}^{n}\right)$. L'inclusion $\operatorname{Rad}(I) \subset$ $\mathcal{I}(\mathcal{Z}(I))$ est évidente. Pour l'inclusion inverse on considère $g \in \mathcal{I}(\mathcal{Z}(I))$. D'après le Théorème 5.21, il existe $f \in I$ telle que $\operatorname{Rad}(f)=\operatorname{Rad}(I)$ et 
$\mathcal{Z}(f)=\mathcal{Z}(I)$. Par conséquent $g \in \mathcal{I}(\mathcal{Z}(f))$ i.e. $\mathcal{Z}(f) \subseteq \mathcal{Z}(g)$. D'après le Lemme 5.1, il existe un entier naturel $N$ tel que l'extension par 0 de $\frac{g^{N}}{f}$ sur $\mathcal{Z}(g)$ soit $k$-régulue sur $\mathbf{R}^{n}$. Notons $h$ cette extension. On obtient finalement $g^{N}=f h \in I$ i.e. $g \in \operatorname{Rad}(I)$.

Remarque 5.25. Soient $n$ et $k$ des entiers naturels. Il est facile de voir que si $F$ est un fermé $k$-régulu de $\mathbf{R}^{n}$ alors $\mathcal{Z}(\mathcal{I}(F))=F$. En effet, $F \subset \mathcal{Z}(\mathcal{I}(F))$ pour tout sous-ensemble de $\mathbf{R}^{n}$ et en écrivant $F=\mathcal{Z}(I)$ pour un idéal $I$ de $\mathcal{R}^{k}\left(\mathbf{R}^{n}\right)$, on a $I \subset \mathcal{I}(\mathcal{Z}(I))$ et

$$
\mathcal{Z}(\mathcal{I}(F))=\mathcal{Z}(\mathcal{I}(\mathcal{Z}(I))) \subset \mathcal{Z}(I)=F .
$$

Le Théorème 5.24 établit donc une correspondance entre les fermés $k$-régulus de $\mathbf{R}^{n}$ et les idéaux radicaux de $\mathcal{R}^{k}\left(\mathbf{R}^{n}\right)$.

Remarquons que l'inégalité de Łojasiewicz 5.1 pour les fonctions $k$-régulues est l'outil principal pour la démonstration du Nullstellensatz 5.24. Ce même type d'idée est utilisé dans [7] pour montrer que l'anneau des fonctions semialgébriques continues est un anneau de Gelfand.

Le spectre de l'anneau des fonctions $k$-régulues sur $\mathbf{R}^{n}$. Soit $n$ un entier naturel et $k$ un entier surnaturel. Soit $f \in \mathcal{R}^{k}\left(\mathbf{R}^{n}\right)$. L'ensemble des zéros de $f$ dans le spectre $\operatorname{Spec} \mathcal{R}^{k}\left(\mathbf{R}^{n}\right)$ est noté $\mathcal{V}(f)$, i.e.,

$$
\mathcal{V}(f)=\left\{p \in \operatorname{Spec} \mathcal{R}^{k}\left(\mathbf{R}^{n}\right) \mid f \in p\right\} .
$$

Son complémentaire est noté $\mathcal{U}(f)$. Plus généralement, si $E$ est un sousensemble de $\mathcal{R}^{k}\left(\mathbf{R}^{n}\right)$, l'ensemble des zéros communs dans $\operatorname{Spec} \mathcal{R}^{k}\left(\mathbf{R}^{n}\right)$ des éléments de $E$ est noté $\mathcal{V}(E)$, et son complémentaire est $\mathcal{U}(E)$. Plus précisément,

et

$$
\mathcal{V}(E)=\left\{p \in \operatorname{Spec} \mathcal{R}^{k}\left(\mathbf{R}^{n}\right) \mid E \subseteq p\right\}
$$

$$
\mathcal{U}(E)=\left\{p \in \operatorname{Spec} \mathcal{R}^{k}\left(\mathbf{R}^{n}\right) \mid E \nsubseteq p\right\} .
$$

Il est bien connu que les sous-ensembles de la forme $\mathcal{U}(E)$ constituent une topologie sur Spec $\mathcal{R}^{k}\left(\mathbf{R}^{n}\right)$ [10, Chapitre 1]. L'espace topologique Spec $\mathcal{R}^{k}\left(\mathbf{R}^{n}\right)$ est quasi-compact. Plus généralement, les ouverts de la forme $\mathcal{U}\left(f_{1}, \ldots, f_{m}\right)$ sont quasi-compacts.

Soient $n$ un entier naturel et $k$ un entier surnaturel. Soit $x \in \mathbf{R}^{n}$. On note encore $\mathfrak{M}_{x}$ l'ensemble des fonctions $k$-régulues sur $\mathbf{R}^{n}$ s'annulant en $x$, i.e.,

$$
\mathfrak{M}_{x}=\left\{f \in \mathcal{R}^{k}\left(\mathbf{R}^{n}\right) \mid f(x)=0\right\} .
$$

Il est clair que $\mathfrak{M}_{x}$ est un idéal maximal de $\mathcal{R}^{k}\left(\mathbf{R}^{n}\right)$. Une conséquence du Nullstellensatz (5.24) est que tout idéal maximal de $\mathcal{R}^{k}\left(\mathbf{R}^{n}\right)$ est de cette forme.

Proposition 5.26. Soient $n$ un entier naturel et $k$ un entier surnaturel. Soit $\mathfrak{M}$ un idéal maximal de $\mathcal{R}^{k}\left(\mathbf{R}^{n}\right)$. Il existe un et un seul $x \in \mathbf{R}^{n}$ tel que $\mathfrak{M}_{x}=\mathfrak{M}$.

Démonstration. Regardons d'abord le cas $k=\infty$. L'anneau des fonctions $\infty$ régulues est égal à l'anneau des fonctions régulières sur $\mathbf{R}^{n}$. Ce dernier est la localisation de l'anneau des polynômes $\mathbf{R}\left[x_{1}, \ldots, x_{n}\right]$ par rapport à la partie 
multiplicative des polynômes ne s'annulant pas sur $\mathbf{R}^{n}$. Si $\mathfrak{M}$ est un idéal maximal de $\mathcal{R}^{\infty}\left(\mathbf{R}^{n}\right)$, c'est par conséquent un ideal réel et le Nullstellensatz classique fonctionnne i.e. $\mathcal{I}(\mathcal{Z}(\mathfrak{M}))=\mathfrak{M}[6$, Thm. 4.1.4].

Soit $k$ un entier surnaturel. D'après le Théorème 5.24 si $k$ est fini et ce qui précède si $k$ est infini, $\mathcal{I}(\mathcal{Z}(\mathfrak{M}))=\mathfrak{M}$. En particulier, $\mathcal{Z}(\mathfrak{M})$ est non-vide. Soit $x \in \mathcal{Z}(\mathfrak{M})$. On a donc $\mathfrak{M} \subseteq \mathfrak{M}_{x}$. Par maximalité, on obtient $\mathfrak{M}=\mathfrak{M}_{x}$. Cela montre l'existence de $x$. L'unicité est claire car $\mathfrak{M}_{x} \neq \mathfrak{M}_{y}$ lorsque $x$ et $y$ sont des éléments distincts de $\mathbf{R}^{n}$.

Soit

$$
\iota: \mathbf{R}^{n} \longrightarrow \operatorname{Spec} \mathcal{R}^{k}\left(\mathbf{R}^{n}\right)
$$

l'application définie par $\iota(x)=\mathfrak{M}_{x}$, où $\mathfrak{M}_{x}$ est l'idéal maximal de $\mathcal{R}^{k}\left(\mathbf{R}^{n}\right)$ des fonctions $k$-régulues s'annulant en $x$. L'application $\iota$ est bien continue lorsqu'on considère $\mathbf{R}^{n}$ avec la topologie $k$-régulue. D'après la version faible du Nullstellensatz régulu, $\iota$ est une bijection sur l'ensemble des idéaux maximaux de $\mathcal{R}^{k}\left(\mathbf{R}^{n}\right)$. La version forte du Nullstellensatz (Théorème 5.24) peut se reformuler ainsi :

Théorème 5.27. Soient $n$ et $k$ des entiers naturels. L'application $\iota$ induit une bijection entre $\operatorname{Spec} \mathcal{R}^{k}\left(\mathbf{R}^{n}\right)$ et l'ensemble des sous-ensembles $k$-régulument fermés et irréductibles de $\mathbf{R}^{n}$. Plus précisément, pour tout sous-ensemble fermé $k$-régulument irréductible $X$ de $\mathbf{R}^{n}$ il existe un et un seul idéal premier $p$ de $\mathcal{R}^{k}\left(\mathbf{R}^{n}\right)$ tel que $X=\iota^{-1}(\mathcal{V}(p))$.

Comme précédemment, cet énoncé est faux si $k=\infty$. Par exemple, l'idéal de $\mathbf{R}[x, y]$ engendré par le polynôme irréductible $x^{2}+y^{2}$ est un idéal premier $p$. Or,

$$
\iota^{-1}(\mathcal{V}(p))=O=\iota^{-1}\left(\mathcal{V}\left(\mathfrak{M}_{O}\right)\right) .
$$

Soit $F \subseteq \mathbf{R}^{n}$ un fermé $k$-régulu. Notons par $\tilde{F}$ le plus petit sous-ensemble fermé de $\operatorname{Spec} \mathcal{R}^{k}\left(\mathbf{R}^{n}\right)$ tel que $\iota(F) \subseteq \tilde{F}$, i.e., $\tilde{F}$ est l'adhérence de $\iota(F)$ dans Spec $\mathcal{R}^{k}\left(\mathbf{R}^{n}\right)$ i.e. $\tilde{F}=\mathcal{V}(\iota(F))$.

Lemme 5.28. Soient $n$ un entier naturel et $k$ un entier surnaturel.

(1) Soit $F_{\alpha}, \alpha \in A$ une collection de sous-ensembles $k$-régulument fermés de $\mathbf{R}^{n}$. Alors

$$
\widetilde{\bigcap_{\alpha \in A} F_{\alpha}}=\bigcap_{\alpha \in A} \widetilde{F}_{\alpha}
$$

(2) Soient $F_{1}, \ldots, F_{m}$ des sous-ensembles $k$-régulument fermés de $\mathbf{R}^{n}$. Alors

$$
\left(F_{1} \cup \widetilde{\sim} \cup F_{m}\right)=\widetilde{F}_{1} \cup \cdots \cup \widetilde{F}_{m} .
$$

Démonstration. La deuxième propriété est évidente. La première est valable car $\iota$ est injectif.

Théorème 5.29. Soient $n$ et $k$ des entiers naturels. L'application $\iota$ induit une bijection entre l'ensemble des sous-ensembles fermés de $\operatorname{Spec} \mathcal{R}^{k}\left(\mathbf{R}^{n}\right)$ et l'ensemble des sous-ensembles k-régulument fermés de $\mathbf{R}^{n}$. Plus précisément, pour tout sous-ensemble $k$-régulument fermé $X$ de $\mathbf{R}^{n}$ il existe un et un seul ensemble fermé $Y$ de $\operatorname{Spec} \mathcal{R}^{k}\left(\mathbf{R}^{n}\right)$ tel que $X=\iota^{-1}(Y)$, à savoir $Y=\tilde{X}$. 
Démonstration. Soit $X$ un fermé $k$-régulu dans $\mathbf{R}^{n}$. Posons $Y=\tilde{X}$ et montrons que $\iota^{-1}(Y)=X$. Comme $\mathbf{R}^{n}$ est noethérien, $X$ possède un nombre fini de composantes irréductibles $k$-régulues $F_{1}, \ldots, F_{m}$. D'après le lemme précédent, $Y=\tilde{F}_{1} \cup \cdots \cup \tilde{F}_{m}$. D'après le Théorème 5.27 , on a alors

$$
\iota^{-1}(Y)=\iota^{-1}\left(\widetilde{F}_{1}\right) \cup \cdots \cup \iota^{-1}\left(\widetilde{F}_{m}\right)=F_{1} \cup \cdots \cup F_{m}=X .
$$

Montrons l'unicité de $Y$. Soit $Z$ un sous-ensemble fermé de $\operatorname{Spec} \mathcal{R}^{k}\left(\mathbf{R}^{n}\right)$ tel que $\iota^{-1}(Z)=X$. Comme $Y$ est le plus petit fermé de $\operatorname{Spec} \mathcal{R}^{k}\left(\mathbf{R}^{n}\right)$ contenant $\iota(X)$, on a $Y \subseteq Z$. Montrons l'inclusion inverse. Soit $p \in Z$, et soit $F=\iota^{-1}(\mathcal{V}(p))$. Montrons d'abord que $\iota(F) \subseteq Y$. Soit $x \in F$. On a $\iota(x) \in \mathcal{V}(p)$. Comme $\mathcal{V}(p)$ est contenu dans $Z$, et comme $\iota^{-1}(Z)=X$, on a $x \in X$. On a également $\iota^{-1}(Y)=X$, donc $\iota(x) \in Y$. Cela montre bien que $\iota(F) \subseteq Y$. Or, d'après le Théorème $5.27, F$ est un fermé $k$-régulument irréductible dans $\mathbf{R}^{n}$, et $p \in \tilde{F}$. Il s'ensuit que $p \in Y$. Cela montre l'inclusion $Z \subseteq Y$.

Corollaire 5.30. Soient $n$ un entier naturel et $k$ un entier surnaturel. L'espace topologique $\operatorname{Spec} \mathcal{R}^{k}\left(\mathbf{R}^{n}\right)$ est noethérien.

Démonstration. Si $k$ est fini, c'est une conséquence du théorème précédent. Pour le cas $k=\infty$, il suffit de rappeler que l'anneau $\mathcal{R}^{\infty}\left(\mathbf{R}^{n}\right)$ est noethérien.

Pour $U$ un ouvert régulu de $\mathbf{R}^{n}$, soit $\tilde{U}$ le complémentaire de $\tilde{F}$ dans $\operatorname{Spec} \mathcal{R}^{k}\left(\mathbf{R}^{n}\right)$, où $F$ est le complémentaire de $U$ dans $\mathbf{R}^{n}$.

Dans l'anneau $\mathcal{R}^{\infty}$ des fonctions régulières sur $\mathbf{R}^{2}$, on peut remarquer que $O=\iota^{-1}(\mathcal{V}(x, y))=\iota^{-1}\left(\mathcal{V}\left(x^{2}+y^{2}\right)\right)$ où $(x, y)$ et $\left(x^{2}+y^{2}\right)$ sont deux idéaux premiers distincts de $\mathcal{R}^{\infty}$. Voici un corollaire qui montre bien la différence entre l'anneau des fonctions régulues et l'anneau des fonctions régulières sur $\mathbf{R}^{n}$ :

Corollaire 5.31. Soient $n$ et $k$ des entiers naturels. Soit $U \subseteq \mathbf{R}^{n}$ un ouvert $k$-régulu. Il existe un et un seul ouvert $V$ de $\operatorname{Spec} \mathcal{R}^{k}\left(\mathbf{R}^{n}\right)$ tel que $\iota^{-1}(V)=U$, à savoir $V=\widetilde{U}$.

Là encore, cet énoncé est faux lorsque $k=\infty$, et ce pour presque tous les ouverts lorsque $n \geq 2$ !

Comme conséquence du corollaire précédent, on donne l'énoncé suivant :

Corollaire 5.32. Soient $n$ et $k$ des entiers naturels. Tout ouvert de $\operatorname{Spec} \mathcal{R}^{k}\left(\mathbf{R}^{n}\right)$ est de la forme $\mathcal{U}(f)$.

Dans la suite de ce paragraphe, on identifiera $\mathbf{R}^{n}$ avec son image dans $\operatorname{Spec} \mathcal{R}^{k}\left(\mathbf{R}^{n}\right)$ via l'application $\iota$, pour simplifier.

Notons $\widetilde{\mathcal{R}}^{k}$ le faisceau structural sur le schéma affine $\operatorname{Spec} \mathcal{R}^{k}\left(\mathbf{R}^{n}\right)$. Il induit par restriction un faisceau en anneaux locaux sur $\mathbf{R}^{n}$ que l'on notera encore $\widetilde{\mathcal{R}}^{k}$. Soit $U$ un ouvert $k$-régulu de $\mathbf{R}^{n}$, et $f$ une fonction $k$-régulue sur $\mathbf{R}^{n}$ telle que $\mathcal{D}(f)=U$. D'après le Corollaire 5.31 , on a

$$
\widetilde{\mathcal{R}}^{k}(U)=\mathcal{R}^{k}\left(\mathbf{R}^{n}\right)_{f} .
$$

D'après la Proposition 5.6,

$$
\mathcal{R}^{k}\left(\mathbf{R}^{n}\right)_{f}=\mathcal{R}^{k}(U) .
$$

Il s'ensuit l'énoncé suivant : 
Corollaire 5.33. Soient $n$ un entier naturel et $k$ un entier surnaturel. La restriction du faisceau structural sur $\operatorname{Spec} \mathcal{R}^{k}\left(\mathbf{R}^{n}\right) \grave{a} \mathbf{R}^{n}$ est le faisceau $\mathcal{R}^{k}$ des fonctions $k$-régulues sur $\mathbf{R}^{n}$.

Le cas $k=\infty$ vient du fait suivant. Soit $U$ un ouvert de Zariski de $\mathbf{R}^{n}$. L'anneau des fonctions régulières $\mathcal{R}^{\infty}(U)$ sur $U$ peut s'identifier avec la limite inductive des localisations $\mathcal{R}^{\infty}\left(\mathbf{R}^{n}\right)_{f}$, où $f$ parcourt l'ensemble des fonctions régulières sur $\mathbf{R}^{n}$ qui ne s'annule pas sur $U$.

Faisceaux $k$-régulus quasi-cohérents sur $\mathbf{R}^{n}$. Soient $n$ un entier naturel et $k$ un entier surnaturel. Soit $M$ un $\mathcal{R}^{k}\left(\mathbf{R}^{n}\right)$-module. Le faisceau $\tilde{M}$ sur le schéma $\operatorname{Spec} \mathcal{R}^{k}\left(\mathbf{R}^{n}\right)$ induit encore par restriction un faisceau $\tilde{M}$ sur $\mathbf{R}^{n}$. Là aussi, pour un ouvert $k$-régulu $U$ de $\mathbf{R}^{n}$, on a

$$
\tilde{M}(U)=M_{f}
$$

lorsque $k$ est fini, où $f$ est une fonction $k$-régulue sur $\mathbf{R}^{n}$ avec $\mathcal{D}(f)=U$. Dans le cas $k=\infty$, le module $\tilde{M}(U)$ peut s'identifier avec la limite inductive des localisations $M_{f}$, où $f$ parcourt l'ensemble des fonctions régulières sur $\mathbf{R}^{n}$ qui ne s'annulent pas sur $U$.

Plus généralement, soit $V$ un ouvert $k$-régulu de $\mathbf{R}^{n}$, et $g$ une fonction $k$ régulue sur $\mathbf{R}^{n}$ telle que $\mathcal{D}(g)=V$. Soit $M$ un $\mathcal{R}^{k}(V)$-module. La restriction à $V$ du faisceau $\tilde{M}$ sur $\operatorname{Spec} \mathcal{R}^{k}(V)$ est un faisceau en $\mathcal{R}_{\mid V}^{k}$-modules, encore noté $\tilde{M}$, déterminé lorsque $k$ est fini par

$$
\tilde{M}(U)=M_{f}
$$

pour tout ouvert $k$-régulu $U$ de $V$, et pour toute fonction $k$-régulue $f$ sur $V$ avec $\mathcal{D}(f)=U$. Dans le cas $k=\infty$, le module $\tilde{M}(U)$ peut s'identifier avec la limite inductive des localisations $M_{f}$, où $f$ parcourt l'ensemble des fonctions régulières sur $V$ qui ne s'annulent pas sur $U$.

Un faisceau $k$-régulu sur $\mathbf{R}^{n}$ est un faisceau en $\mathcal{R}^{k}$-modules sur $\mathbf{R}^{n}$. Soit $\mathcal{F}$ un faisceau $k$-régulu sur $\mathbf{R}^{n}$. On dira que le faisceau $\mathcal{F}$ est quasi-cohérent s'il existe un recouvrement de $\mathbf{R}^{n}$ par des ouverts $k$-régulus $U_{i}, i \in I$, tel que, pour tout $i$, il existe un $\mathcal{R}^{k}\left(U_{i}\right)$-module $M_{i}$ avec $\tilde{M}_{i}$ isomorphe à $\mathcal{F}_{\mid U_{i}}$.

Nous obtenons alors une version $k$-régulue du Théorème A de Cartan, cf. [13, Chap. II, Corollary 5.5].

Théorème 5.34. Soient $n$ et $k$ des entiers naturels. Le foncteur qui associe à un $\mathcal{R}^{k}\left(\mathbf{R}^{n}\right)$-module $M$ le faisceau $\tilde{M}$ est une équivalence de catégories sur la catégorie des faisceaux $k$-régulus quasi-cohérents sur $\mathbf{R}^{n}$. Le foncteur réciproque est le foncteur "sections globales" $H^{0}$. En particulier, le faisceau $\tilde{M}$ est engendré par ses sections globales.

Comme signalé dans l'introduction, ce résultat est faux pour $k=\infty$. En effet, il existe des faisceaux réguliers quasi-cohérents sur $\mathbf{R}^{n}$ qui ne sont pas engendrés par leurs sections globales:

Exemple 5.35 (cf.[6, Example 12.1.5]). Soit $p \in \mathbf{R}[x, y]$ le polynôme défini par

$$
p=x^{2}(x-1)^{2}+y^{2} .
$$


Remarquons que $p$ possède exactement deux racines réelles à savoir $c_{0}=(0,0)$ et $c_{1}=(1,0)$. Soit $U_{i}=\mathbf{R}^{2} \backslash\left\{c_{i}\right\}$ pour $i=0,1$. Les ouverts réguliers $U_{0}$ et $U_{1}$ constituent un recouvrement de $\mathbf{R}^{2}$. On définit un faisceau régulier quasicohérent $\mathcal{F}$ par rapport à ce recouvrement. En fait, $\mathcal{F}$ va être localement libre de rang 1. Explicitement, on construit $\mathcal{F}$ à partir des faisceaux $\mathcal{R}_{\mid U_{0}}^{\infty}$ et $\mathcal{R}_{\mid U_{1}}^{\infty}$ en les recollant au-dessus de $U_{0} \cap U_{1}$ à l'aide de la fonction de transition $g_{01}=p$ sur $U_{0} \cap U_{1}$. Plus précisément, deux sections $s_{0}$ et $s_{1}$ de $\mathcal{R}_{\mid U_{0}}^{\infty}$ et $\mathcal{R}_{\mid U_{1}}^{\infty}$ audessus d'ouverts de Zariski $V_{0}$ et $V_{1}$, respectivement, se recollent si $g_{01} s_{1}=s_{0}$ $\operatorname{sur} V_{0} \cap V_{1}$.

Montrons que toute section régulière globale $s$ de $\mathcal{F}$ s'annule en $c_{1}$. La restriction $s_{i}$ de $s$ à $U_{i}$ est une fonction régulière sur $U_{i}$, pour $i=0,1$. La condition de recollement est $g_{01} s_{1}=s_{0}$ sur $U_{0} \cap U_{1}$. Ecrivons $s_{i}=p_{i} / q_{i}$, où $p_{i}, q_{i} \in \mathbf{R}[x, y]$, avec $q_{i} \neq 0$ sur $U_{i}$ et $p_{i}, q_{i}$ premiers entre eux, pour $i=0,1$. La condition de recollement implique que $p q_{0} p_{1}=p_{0} q_{1}$ sur $\mathbf{R}^{2}$. Comme $p$ est irréductible, et comme $q_{1}\left(c_{0}\right) \neq 0$, le polynôme $p$ divise $p_{0}$. En particulier, $p_{0}\left(c_{1}\right)=0$ et donc aussi $s\left(c_{1}\right)=0$.

Il s'ensuit que le faisceau régulier quasi-cohérent $\mathcal{F}$ sur $\mathbf{R}^{2}$ n'est pas engendré par ses sections globales.

Pour compléter, montrons que le faisceau $k$-régulu quasi-cohérent $\mathcal{F}^{k}=$ $\mathcal{R}^{k} \otimes \mathcal{F}$ induit est bien engendré par ses sections globales, pour $k$ un entier naturel. On montre même plus : le faisceau $\mathcal{F}^{k}$ est isomorphe à $\mathcal{R}^{k}$ en exhibant une section globale de $\mathcal{F}^{k}$ qui ne s'annule jamais. Soit $s$ la section régulue globale de $\mathcal{F}^{k}$ définie par

$$
s_{0}=\frac{1}{\left(x^{2}+y^{2}\right)^{\ell}} \quad \text { et } \quad s_{1}=\frac{\left(x^{2}+y^{2}\right)^{\ell}}{p},
$$

où $\ell$ est le plus petit entier $\geq(k+1) / 2$. La fonction $s_{i}$ est bien $k$-régulue sur $U_{i}$ et non nulle, pour $i=0,1$. Le faisceau $k$-régulu $\mathcal{F}^{k}$ est donc isomorphe à $\mathcal{R}^{k}$. En particulier, il est engendré par ses sections globales.

Il suit du Théorème A de Cartan version $k$-régulue, que le foncteur "sections globales" est exact sur les faisceaux $k$-régulus quasi-cohérents sur $\mathbf{R}^{n}$. D'où la version $k$-régulue du Théorème $\mathrm{B}$ de Cartan, cf. [11, Théorème 1.3.1]. (On se réfère ici à EGAIII car le schéma $\operatorname{Spec} \mathcal{R}^{k}\left(\mathbf{R}^{n}\right)$ n'est pas noethérien d'après la Proposition 4.16 or la preuve donnée de [13, Chap. III, Theorem 3.5] n'est valable que pour les schémas noethériens).

Théorème 5.36. Soient $n$ et $k$ des entiers naturels. Soit $\mathcal{F}$ un faisceau $k$ régulu quasi-cohérent sur $\mathbf{R}^{n}$. Alors,

$$
H^{i}\left(\mathbf{R}^{n}, \mathcal{F}\right)=0
$$

pour tout $i \neq 0$.

Variétés $k$-régulues affines. Soit $F$ un sous-ensemble $k$-régulument fermé de $\mathbf{R}^{n}$. La topologie $k$-régulue sur $F$ est la topologie induite par la topologie $k$ régulue de $\mathbf{R}^{n}$. Soit $\mathcal{I}$ le faisceau d'idéaux de $\mathcal{R}^{k}$ des fonctions s'annulant sur $F$. Le faisceau quotient $\mathcal{R}^{k} / \mathcal{I}$ possède un support égal à $F$, et est donc un faisceau sur $F$, le faisceau des fonctions $k$-régulues sur $F$, noté $\mathcal{R}_{F}^{k}$. Soit $U$ un ouvert $k$-régulu de $F$. Une fonction $k$-régulue sur $F$ est une section du faisceau $\mathcal{R}_{F}^{k}$. En 
particulier, une fonction $k$-régulue sur $F$ est une section globale du faisceau $\mathcal{R}_{F}^{k}$ sur $F$.

Lorsque $F \subset \mathbf{R}^{n}$ est une variété réelle algébrique affine lisse, cette définition est a priori plus forte que la Définition 2.15. En effet, tout élément de $\mathcal{R}_{F}^{k}(F)$ est une fonction $k$-régulue au sens de 2.15 . Pour $k=0$, la réciproque est vraie d'après [19]. Pour un entier naturel $k \neq 0$, la question est ouverte.

Proposition 5.37. Soient $n$ un entier naturel et $k$ un entier surnaturel. Soit $F$ un fermé $k$-régulu de $\mathbf{R}^{n}$, et $U$ un ouvert $k$-régulu de $F$. Soit $V$ un ouvert $k$ régulu de $\mathbf{R}^{n}$ tel que $V \cap F=U$. Si $f$ est une fonction $k$-régulue sur $U$, alors il existe une fonction $k$-régulue $g$ sur $V$ dont la restriction à $U$ est égal à $f$.

Démonstration. Montrons l'énoncé d'abord dans le cas où $k$ est fini. On a une suite exacte de faisceaux quasi-cohérents

$$
0 \rightarrow \mathcal{I}_{\mid V} \rightarrow \mathcal{R}_{\mid V}^{k} \rightarrow\left(\mathcal{R}_{F}^{k}\right)_{\mid V} \rightarrow 0
$$

sur $\mathbf{R}^{n}$. D'après la version $k$-régulue du Théorème $\mathrm{B}$ de Cartan, on en déduit une suite exacte

$$
0 \longrightarrow \mathcal{I}(V) \rightarrow \mathcal{R}^{k}(V) \rightarrow \mathcal{R}_{F}^{k}(V) \rightarrow 0 .
$$

Comme $\mathcal{R}_{F}^{k}(V)=\mathcal{R}_{F}^{k}(U)$, il existe bien une fonction $k$-régulue $g$ sur $V$ dont la restriction à $U$ est égale à $f$, pour toute fonction $f k$-régulue sur $U$, lorsque $k$ est fini.

Il nous reste à montrer l'énoncé lorsque $k=\infty$. Soit $f$ une fonction régulière sur $U$. Il existe des fonctions polynomiales $p$ et $q$ sur $\mathbf{R}^{n}$ telles que $f(x)=p(x) / q(x)$ pour tout $x \in U$. Soit $s$ une fonction polynomiale sur $\mathbf{R}^{n}$ dont l'ensemble des zéros est égal à $F$. L'ensemble des zéros de la fonction polynomiale $q^{2}+s^{2}$ est contenu dans le fermé de Zariski $G=F \backslash U$. La fonction définie sur $\mathbf{R}^{n} \backslash G$ par

$$
g(x)=\frac{p(x) q(x)}{q^{2}(x)+s^{2}(x)}
$$

est bien régulière, et sa restriction à $U$ est égale à $f$.

Corollaire 5.38. Soient $n$ un entier naturel et $k$ un entier surnaturel. Soit $F$ un fermé $k$-régulu de $\mathbf{R}^{n}$. Si $f$ est une fonction $k$-régulue sur $F$, alors il existe une fonction $k$-régulue $g$ sur $\mathbf{R}^{n}$ dont la restriction à $F$ est égale à $f$. Plus précisément, l'application de restriction de $\mathcal{R}^{k}\left(\mathbf{R}^{n}\right)$ dans $\mathcal{R}^{k}(F)$ induit un isomorphisme

$$
\mathcal{R}^{k}\left(\mathbf{R}^{n}\right) / \mathcal{I}(F) \cong \mathcal{R}^{k}(F) .
$$

Remarque 5.39. D'après le corollaire précédent, les fonctions $k$-régulues telles que nous venons de les définir sur des sous-ensembles fermés $k$-régulus coïncident avec les fonctions «hereditarily rational» de Kollár [19], lorsque $k=0$. L'exemple [19, Ex. 2] d'une fonction rationnelle continue sur une surface singulière dont la restriction à l'axe des $z$ n'est pas rationnelle n'est pas une fonction régulue.

Soit $F$ un sous-ensemble $k$-régulument fermé de $\mathbf{R}^{n}$. La paire $\left(F, \mathcal{R}_{F}^{k}\right)$ est un espace localement annelé en $\mathbf{R}$-algèbres. Une variété $k$-régulue affine est un espace localement annelé en $\mathbf{R}$-algèbres isomorphe à $\left(F, \mathcal{R}_{F}^{k}\right)$ pour un certain 
fermé $k$-régulu $F$ de $\mathbf{R}^{n}$, où $n$ est un entier naturel. Un morphisme entre variétés $k$-régulues affines est un morphisme d'espaces localement annelés en R-algèbres.

Corollaire 5.40. Soient $m$ et $n$ des entiers naturels et $k$ un entier surnaturel. Soient $F \subseteq \mathbf{R}^{n}$ et $G \subseteq \mathbf{R}^{m}$ des fermés k-régulus. Une application $f: G \rightarrow F$ est un morphisme de variétés affines $k$-régulues si et seulement s'il existe des fonctions $k$-régulues $f_{1}, \ldots, f_{n}$ sur $\mathbf{R}^{m}$ telles que

$$
f(x)=\left(f_{1}(x), \ldots, f_{n}(x)\right)
$$

pour tout $x \in G$.

Soit $k$ un entier surnaturel. Soit $(X, \mathcal{O})$ une variété $k$-régulue affine. Soit $f$ une fonction $k$-régulue sur $X$, i.e., $f$ est une section globale de $\mathcal{O}$. L'ensemble des zéros de $f$ dans le spectre $\operatorname{Spec} \mathcal{O}(X)$ est noté $\mathcal{V}(f)$, i.e.,

$$
\mathcal{V}(f)=\{p \in \operatorname{Spec} \mathcal{O}(X) \mid f \in p\} .
$$

Son complémentaire est noté $\mathcal{U}(f)$. Plus généralement, si $E$ est un sousensemble de $\mathcal{O}(X)$, l'ensemble des zéros communs dans $\operatorname{Spec} \mathcal{O}(X)$ des éléments de $E$ est noté $\mathcal{V}(E)$, et son complémentaire est $\mathcal{U}(E)$. Plus précisément,

$$
\mathcal{V}(E)=\{p \in \operatorname{Spec} \mathcal{O}(X) \mid E \subseteq p\}
$$

et

$$
\mathcal{U}(E)=\left\{p \in \operatorname{Spec} \mathcal{R}^{k}(F) \mid E \nsubseteq p\right\} .
$$

Comme précédemment, les sous-ensembles de la forme $\mathcal{U}(E)$ constituent une topologie sur $\operatorname{Spec} \mathcal{O}(X)$ [10, Chapitre 1]. L'espace topologique $\operatorname{Spec} \mathcal{O}(X)$ est quasi-compact. Plus généralement, les ouverts de la forme $\mathcal{U}\left(f_{1}, \ldots, f_{m}\right)$ sont quasi-compacts.

Soit $x \in X$. On note $\mathfrak{M}_{x}$ l'idéal maximal de $\mathcal{O}(X)$ des fonctions $k$-régulues sur $X$ s'annulant en $x$. La Proposition 5.26 implique alors l'énoncé suivant :

Proposition 5.41. Soit $k$ un entier surnaturel. Soit $X$ une variété $k$-régulue affine. Soit $\mathfrak{M}$ un idéal maximal de $\mathcal{O}(X)$. Il existe un et un seul $x \in X$ tel que $\mathfrak{M}_{x}=\mathfrak{M}$.

Comme dans le cas $\mathbf{R}^{n}$, on a une injection

$$
\iota: X \longrightarrow \operatorname{Spec} \mathcal{O}(X) \text {. }
$$

Le Théorème 5.27 se généralise alors ainsi :

Théorème 5.42. Soit $k$ un entier naturel. Soit $X$ une variété $k$-régulue affine. L'application ı ci-dessus induit une bijection entre l'ensemble des sousensembles fermés de $\operatorname{Spec} \mathcal{O}(X)$ et l'ensemble des sous-ensembles fermés de $X$. Plus précisément, pour tout sous-ensemble fermé $F$ de $X$ il existe un et un seul ensemble fermé $G$ de $\operatorname{Spec} \mathcal{O}(X)$ tel que $F=\iota^{-1}(G)$.

Pour les ouverts cela donne l'énoncé suivant :

Corollaire 5.43. Soit $k$ un entier naturel. Soit $X$ une variété $k$-régulue affine. Soit $U \subseteq X$ un ouvert. Il existe un et un seul ouvert $V$ de $\operatorname{Spec} \mathcal{O}(X)$ tel que $\iota^{-1}(V)=U$. 
On a encore :

Corollaire 5.44. Soit $k$ un entier naturel. Soit $X$ une variété $k$-régulue affine. Tout ouvert de $\operatorname{Spec} \mathcal{O}(X)$ est de la forme $\mathcal{U}(f)$, pour une certaine fonction $k$-régulue sur $X$.

Comme auparavant, on identifiera $X$ avec son image dans $\operatorname{Spec} \mathcal{O}(X)$ via l'application $\iota$.

Corollaire 5.45. Soit $k$ un entier surnaturel. Soit $X$ une variété $k$-régulue affine. La restriction du faisceau structural sur $\operatorname{Spec} \mathcal{O}(X)$ à $X$ s'identifie avec le faisceau $\mathcal{O}$.

Soit $M$ un $\mathcal{O}(X)$-module. Le faisceau $\tilde{M}$ sur le schéma $\operatorname{Spec} \mathcal{O}(X)$ induit encore par restriction un faisceau $\tilde{M}$ sur $X$. Pour un ouvert $U$ de $X$, on a

$$
\tilde{M}(U)=M_{f}
$$

lorsque $k$ est fini, où $f$ est une fonction $k$-régulue sur $X$ avec $\mathcal{D}(f)=U$.

Un faisceau $k$-régulu sur $X$ est un faisceau en $\mathcal{O}$-modules sur $X$. Soit $\mathcal{F}$ un faisceau $k$-régulu sur $X$. On dira que le faisceau $\mathcal{F}$ est quasi-cohérent s'il existe un recouvrement de $X$ par des ouverts $k$-régulus $U_{i}, i \in I$, tels que, pour tout $i$, il existe un $\mathcal{O}\left(U_{i}\right)$-module $M_{i}$ avec $\tilde{M}_{i}$ isomorphe à $\mathcal{F}_{\mid U_{i}}$.

La version $k$-régulue du Théorème $\mathrm{A}$ de Cartan implique alors l'énoncé suivant :

Théorème 5.46. Soit $k$ un entier naturel. Soit $X$ une variété $k$-régulue affine. Le foncteur qui associe à un $\mathcal{O}(X)$-module $M$ le faisceau $\tilde{M}$ est une équivalence de catégories sur la catégorie des faisceaux k-régulus quasi-cohérents sur $X$. Le foncteur réciproque est le foncteur "sections globales" $H^{0}$. En particulier, le faisceau $\tilde{M}$ est engendré par ses sections globales.

De même, on a une version $k$-régulue du Théorème B de Cartan pour les variétés $k$-régulues affines :

Théorème 5.47. Soit $k$ un entier naturel. Soit $X$ une variété $k$-régulue affine. Soit $\mathcal{F}$ un faisceau k-régulu quasi-cohérent sur $X$. Alors,

$$
H^{i}(X, \mathcal{F})=0
$$

pour tout $i \neq 0$.

En particulier les théorèmes 5.46 et 5.47 s'appliquent pour tout $k \in \mathbf{N}$ lorsque $X$ est une variété réelle algébrique affine singulière. L'énoncé est alors en tout point identique au cas algébriquement clos! Cf. [13, Chap. III].

\section{EnsEMbles RÉGULUMENT FERMÉS}

Le but de cette section est de déterminer les sous-ensembles $k$-régulument fermés de $\mathbf{R}^{n}$ pour $k$ et $n$ des entiers naturels. Ceci fait, si $F$ est un sousensemble $k$-régulument fermé de $\mathbf{R}^{n}$ (par exemple si $F$ est une sous-variété réelle algébrique de $\mathbf{R}^{n}$ ), on saura caractériser les sous-ensembles $k$-régulument fermés de $F$ via la topologie induite. 
Ensembles algébriquement constructibles. On rappelle qu'un sous-ensemble semi-algébrique de $\mathbf{R}^{n}$ est algébriquement constructible s'il appartient à l'algèbre booléenne engendré par les sous-ensembles Zariski fermés de $\mathbf{R}^{n}$. Ces ensembles forment une catégorie constructible, de la même manière que les ensembles symétriques par arcs. On renvoie à $[28,26]$ pour une introduction aux catégories constructibles.

Le but de cette section est de montrer que les sous-ensembles $k$-régulument fermés de $\mathbf{R}^{n}$ coïncident avec les sous-ensembles algébriquement constructibles fermés de $\mathbf{R}^{n}$. On commence par deux résultats précisant la place des points réguliers ainsi que la dimension d'un ensemble $k$-régulument fermé vis-à-vis de son adhérence de Zariski.

Si $E$ est un sous-ensemble de $\mathbf{R}^{n}$, on notera dans la suite $\bar{E}^{\text {eucl }}$ l'adhérence de $E$ dans $\mathbf{R}^{n}$ pour la topologie euclidienne.

Théorème 6.1. Soit $I$ un idéal premier de $\mathcal{R}^{0}\left(\mathbf{R}^{n}\right)$. On note $J=I \cap$ $\mathbf{R}\left[x_{1}, \ldots, x_{n}\right]$ et on suppose que $J$ est principal, i.e. $J=(s)$ avec $s \in$ $\mathbf{R}\left[x_{1}, \ldots, x_{n}\right]$ irréductible. On note $V$ l'ensemble $\mathcal{Z}(J)$ et $V_{\text {reg }}$ l'ensemble des points lisses de $V$. Alors

$$
{\overline{V_{\text {reg }}}}^{\text {eucl }} \subseteq \mathcal{Z}(I)=\left\{x \in \mathbf{R}^{n} \mid I \subseteq \mathfrak{M}_{x}\right\}=\left\{x \in \mathbf{R}^{n} \mid f(x)=0, \forall f \in I\right\} .
$$

Démonstration. Les fonctions de $\mathcal{R}^{0}\left(\mathbf{R}^{n}\right)$ étant continues, il est suffisant de montrer que

$$
V_{\text {reg }} \subseteq \mathcal{Z}(I)=\left\{x \in \mathbf{R}^{n} \mid I \subseteq \mathfrak{M}_{x}\right\} .
$$

Pour $x \in V_{\text {reg }}$, on doit montrer que $I \subseteq \mathfrak{M}_{x}$. Supposons l'existence de $f \in I$ telle que $f \notin \mathfrak{M}_{x}$, c'est-à-dire $f(x) \neq 0$. On écrit $f=\frac{p}{q}$ avec $p, q \in \mathbf{R}\left[x_{1}, \ldots, x_{n}\right]$ premiers entre eux. On obtient alors

$$
p=q . f \in I \cap \mathbf{R}\left[x_{1}, \ldots, x_{n}\right]=J=(s)
$$

et donc $p=r . s$ avec $r \in \mathbf{R}\left[x_{1}, \ldots, x_{n}\right]$. Ceci implique que $p$ s'annule identiquement sur $V$, i.e. $V \subseteq \mathcal{Z}(p)$. Le Lemme 5.9 nous montre que $J=(s)$ est un idéal réel et par conséquent $\operatorname{dim}(V=\mathcal{Z}(s))=n-1$ [6, Thm. 4.5.1]. On en déduit que $\operatorname{dim} \mathcal{Z}(p)=n-1$. Comme $\mathcal{Z}(q) \subseteq \mathcal{Z}(p)$ puisque $f \in \mathcal{R}^{0}\left(\mathbf{R}^{n}\right)$, on a aussi $q(x)=0$ car $f(x) \neq 0$. On sait que $\operatorname{dim} \mathcal{Z}(q) \leq n-2$ par la Proposition 3.5. Comme $x \in V_{\text {reg }}$, il existe un voisinage ouvert semi-algébrique de $x$ dans $V \subseteq \mathcal{Z}(p)$ de dimension $n-1$ [6, Prop. 3.3.10]. On note $A$ le semi-algébrique $\mathcal{Z}(p) \backslash \mathcal{Z}(q)$. D'après ce qu'on a dit précédemment, le point $x$ est un point adhérent à $A$. Le lemme de sélection des courbes [6, Thm. 2.5.5] pour le semialgébrique $A$ nous fournit une fonction semi-algébrique continue $h:[0,1] \rightarrow \mathbf{R}^{n}$ telle que $h(0)=x$ et $h(] 0,1]) \subseteq A$. Par conséquent $f \circ h:[0,1] \rightarrow \mathbf{R}$ est une fonction semi-algébrique continue telle que $(f \circ h)([0,1])=0$ et $(f \circ h)(0) \neq 0$, ce qui contredit la continuité de $f$.

On évalue maintenant la dimension d'un fermé régulu vu comme un ensemble semi-algébrique, c'est-à-dire la dimension de son adhérence de Zariski.

Proposition 6.2. Soit I un idéal radical de $\mathcal{R}^{0}\left(\mathbf{R}^{n}\right)$ et $J=I \cap \mathbf{R}\left[x_{1}, \ldots, x_{n}\right]$ sa trace sur les polynômes. On note $V$ l'ensemble $\mathcal{Z}(J)$. Alors

$$
\overline{\mathcal{Z}(I)}^{\text {Zar }}=V
$$


où $\overline{\mathcal{Z}(I)}^{\text {Zar }}$ est l'adhérence pour la topologie de Zariski de $\mathcal{Z}(I)$. En particulier $\operatorname{dim} \mathcal{Z}(I)=\operatorname{dim} V$.

Démonstration. Posons $V^{\prime}=\overline{\mathcal{Z}(I)}^{\text {Zar }}$. Alors $V^{\prime}$ est inclus dans $V$ car $V$ est un fermé de Zariski contenant $\mathcal{Z}(I)$.

Supposons maintenant que $V^{\prime}$ soit strictement inclus dans $V$. Alors $\mathcal{I}\left(V^{\prime}\right)=$ $\mathcal{I}(\mathcal{Z}(I))$ contient strictement $\mathcal{I}(V)$, sinon $\mathcal{Z}\left(\mathcal{I}\left(V^{\prime}\right)\right)=V^{\prime}=\mathcal{Z}(\mathcal{I}(V))=V$. Par conséquent il existe $p \in \mathcal{I}\left(V^{\prime}\right)$ tel que $p \notin \mathcal{I}(V)$. Mais $p$ est une fonction régulue qui s'annule identiquement sur $\mathcal{Z}(I)$ et donc, par le Nullstellensatz régulu,

$$
p \in \mathcal{I}_{\mathcal{R}^{0}}(\mathcal{Z}(I)) \cap \mathbf{R}\left[x_{1}, \ldots, x_{n}\right]=I \cap \mathbf{R}\left[x_{1}, \ldots, x_{n}\right]=J .
$$

Par conséquent $p(V)=0$, et donc $p$ appartient à $\mathcal{I}(V)$, en contradiction avec le choix de $p$.

Dans la cas du plan, on peut construire à la main une fonction régulue qui sépare les points isolés de l'adhérence euclidienne des points réguliers d'une courbe, démontrant au passage que l'adhérence au sens de la topologie euclidienne des points réguliers de la courbe est un ensemble régulument fermé.

Proposition 6.3. Soit I un idéal premier de $\mathcal{R}^{0}\left(\mathbf{R}^{2}\right)$. Soit $J=I \cap \mathbf{R}\left[x_{1}, x_{2}\right]$ et notons $V$ l'ensemble $\mathcal{Z}(J)$. On note aussi $V_{\text {reg }}$ l'ensemble des points lisses de $V$ et $W={\overline{V_{\text {reg }}}}^{\text {eucl }}$. Alors

$$
W=\mathcal{Z}(I)=\left\{x \in \mathbf{R}^{2} \mid I \subseteq \mathfrak{M}_{x}\right\}=\left\{x \in \mathbf{R}^{2} \mid f(x)=0 \forall f \in I\right\} .
$$

Démonstration. Si $J=(0)$ alors $I=(0)$ et le résultat est évident. Si $J$ est maximal, le Corollaire 5.26 donne le résultat voulu.

On suppose donc que $J$ est de hauteur 1, c'est-à-dire $J=(s)$ avec $s \in \mathbf{R}\left[x_{1}, x_{2}\right]$ irréductible. Ainsi $V=\mathcal{Z}(s)$ est une courbe algébrique affine irréductible. L'idéal $J$ étant réel, on a $\mathcal{I}(\mathcal{Z}(J))=J$. L'inclusion

$$
W \subseteq \mathcal{Z}(I)
$$

provient du Théorème 6.1. Comme $J \subseteq I$, on obtient par ailleurs $\mathcal{Z}(I) \subseteq$ $\mathcal{Z}(J)=V$. En résumé, on a les inclusions suivantes

$$
W \subseteq \mathcal{Z}(I) \subseteq V .
$$

Pour terminer la preuve, pour tout $x \in V \backslash W$ on doit trouver $f \in I$ telle que $f(W)=0$ et vérifiant $f(x) \neq 0$.

Soit $x \in V \backslash W$. Supposons $x$ égal à l'origine $O$ pour simplifier les notations. Alors $O$ est un zéro isolé de $s$. Il existe donc un disque fermé $B$ centré en $O$ tel que $O$ soit l'unique zéro de $s$ dans $B$. En utilisant l'inégalité de Łojasiewicz pour $x_{1}^{2}+x_{2}^{2}$ et $\frac{1}{s^{2}}$ dans $B$, il existe un entier strictement positif $N$ tel que $\frac{\left(x_{1}^{2}+x_{2}^{2}\right)^{N}}{s^{2}}$ s'étende de façon continue par 0 en $O$ dans $B$. On pose $f=\frac{s^{2}}{s^{2}+\left(x_{1}^{2}+x_{2}^{2}\right)^{2 N}}$. La fonction $f$ est clairement continue en dehors de $O$ et peut être étendue de manière continue par 1 en $O$ car

$$
\lim _{\left(x_{1}, x_{2}\right) \rightarrow O} \frac{\left(x_{1}^{2}+x_{2}^{2}\right)^{2 N}}{s^{2}}=0 .
$$


Par conséquent $f \in \mathcal{R}^{0}\left(\mathbf{R}^{2}\right)$ et la restriction de $f$ à $V_{\text {reg }}$ est identiquement nulle puisque la restriction de $f$ à $V \backslash\{O\}$ est identiquement nulle. Comme $f$ est continue on a aussi $f(W)=0$. Il reste donc à montrer que $f$ appartient à I. Déjà

$$
\left(s^{2}+\left(\left(x_{1}^{2}+x_{2}^{2}\right)^{N}\right)^{2}\right) \cdot f \in I,
$$

et puisque $I$ est un idéal premier, au moins un des deux termes du produit appartient à $I$. Supposons qu'il s'agisse du premier, c'est-à-dire

$$
\left(s^{2}+\left(\left(x_{1}^{2}+x_{2}^{2}\right)^{N}\right)^{2}\right) \in I \cap \mathbf{R}\left[x_{1}, x_{2}\right]=J .
$$

Alors $\left(x_{1}^{2}+x_{2}^{2}\right)^{N}$ appartient à $J$ car $J$ est un idéal réel. L'idéal $J$ étant de plus premier, on en déduit que $x_{1}^{2}+x_{2}^{2} \in J$. Mais alors $x_{1} \in J$ et $x_{2} \in J$ en invoquant une nouvelle fois la réalité de $J$. On en déduit que $J$ est maximal, en contradiction avec notre hypothèse. Par conséquent $f$ appartient à $I$, ce qui termine la preuve de la proposition.

En particulier, on constate que les courbes 0-régulument fermées du plan sont des ensembles Zariski constructibles fermés. On montre ci-dessous que c'est en fait le cas pour tout ensemble $k$-régulu.

Théorème 6.4. Soit $k$ un entier naturel. Les sous-ensembles $k$-régulument fermés de $\mathbf{R}^{n}$ coincident avec les sous-ensembles algébriquement constructibles fermés de $\mathbf{R}^{n}$.

Démonstration. On sait déjà que tout ensemble $k$-régulument fermé de $\mathbf{R}^{n}$ est algébriquement constructible d'après le Corollaire 4.12. On montre la réciproque par récurrence sur la dimension.

En dimension nulle, le résultat est clair. Soit $C$ un sous-ensemble algébriquement constructible fermé de $\mathbf{R}^{n}$. On peut supposer que $C$ est irréductible, autrement dit que son adhérence de Zariski $V$ est une sous-variété réelle algébrique irréductible de $\mathbf{R}^{n}$, quitte à raisonner composante par composante. On note $Z$ la réunion des composantes algébriquement constructibles de $V$ de dimension strictement plus petite que $V$. Ainsi $V=C \cup Z$. Il existe une fonction $k$-régulue $g$ définie sur $\mathbf{R}^{n}$ telle que $\mathcal{Z}(g)=Z$ par hypothèse de récurrence. Par ailleurs, soit $f$ une fonction régulière sur $\mathbf{R}^{n}$ telle que $\mathcal{Z}(f)=V$. D'après le Lemme 5.2, il existe un entier naturel $N$ tel que la fonction $\frac{g^{N}}{f^{2}}, k$-régulue sur $\mathcal{D}(f)$, se prolonge par 0 sur $\mathcal{D}(f) \cup Z \backslash C$ en une fonction $k$-régulue. Posons

$$
h=\frac{f^{2}}{f^{2}+g^{2 N}} .
$$

Alors la fonction $h$ est $k$-régulue sur $\mathcal{D}(g)$, et s'annule sur $C \cap \mathcal{D}(g)$. On la prolonge par 1 sur $Z$. Elle est alors $k$-régulue en dehors de $Z \cap C$ par choix de $N$. L'ensemble $Z \cap C$ est algébriquement constructible de dimension strictement plus petite que $C$, il existe donc une fonction $k$-régulue $l$ telle que $Z \cap C=\mathcal{Z}(l)$ par hypothèse de récurrence. D'après le Lemme 5.1, il existe alors un entier $N^{\prime}$ tel que la fonction $l^{N^{\prime}} h$ soit $k$-régulue sur $\mathbf{R}^{n}$. Elle satisfait $\mathcal{Z}\left(l^{N^{\prime}} h\right)=C$, ce qui fait de $C$ un ensemble $k$-régulument fermé.

Corollaire 6.5. Pour $k$ et $k^{\prime}$ des entiers naturels, les topologies $k$-régulue et $k^{\prime}$-régulue sont équivalentes. 
Décomposition en ensembles symétriques par arcs. On sait qu'un sous-ensemble $k$-régulument fermé de $\mathbf{R}^{n}$ est un sous-ensemble algébriquement constructible fermé d'après le Théorème 6.4. En particulier, un ensemble $k$-régulument fermé irréductible est égal à la composante algébriquement constructible de dimension maximale de son adhérence de Zariski. Malheureusement, il est difficile de décrire géométriquement cette composante. Le but de cette section est de décrire cette composante algébriquement constructible de dimension maximale en terme d'ensembles symétriques par arcs.

Proposition 6.6. Soit $V$ une sous-variété algébrique irréductible de $\mathbf{R}^{n}$ de dimension $d$. Soit $S$ un sous-ensemble semi-algébrique de $V$ de dimension $d$ et soit $f \in \mathcal{R}^{0}\left(\mathbf{R}^{n}\right)$ s'annulant identiquement sur $S$. Alors $f$ s'annule identiquement sur $V_{\text {reg }}$ et donc sur la réunion $W$ des composantes $\mathcal{A} \mathcal{R}$ irréductibles de dimension maximale de $V$.

Pour autant, il se peut que $f$ s'annule sur strictement plus que $W$ comme on le verra dans l'exemple 6.10.

Démonstration de la Proposition 6.6. La fonction $f$ reste régulue en restriction à $V$ (cf. Remarque 5.39). Il existe donc une composition d'éclatements à centres lisses $\phi: \tilde{V} \rightarrow V$, avec $\tilde{V}$ une variété réelle algébrique irréductible non singulière, telle que $f \circ \phi$ soit régulière sur $\tilde{V}$ d'après le Théorème 3.11.

Comme $S$ est de dimension $d$, la fonction régulière $f \circ \phi$ s'annule sur un sousensemble Zariski dense de $\tilde{V}$, donc sur $\tilde{V}$ qui est irréductible. Alors $f$ s'annule sur les points réguliers de $V$, donc aussi sur $W$ car $Z(f)$ est un ensemble symétrique par arcs.

Soit $E$ un ensemble semi-algébrique de $\mathbf{R}^{n}$. D'après [26, Cor. 2.15], l'intersection des ensembles symétriques par arcs contenant $E$ est un ensemble symétrique par arcs de même dimension que $E$, et noté $\bar{E}^{\mathcal{A} \mathcal{R}}$. De même, on note $\bar{E}^{C}$ l'adhérence de $E$ pour la topologie algébriquement constructible (identique à la topologie régulue comme on vient de le voir).

On généralise maintenant le Théorème 6.1 au cas non principal.

Théorème 6.7. Soit $I$ un idéal premier de $\mathcal{R}^{0}\left(\mathbf{R}^{n}\right)$. On note $J=I \cap$ $\mathbf{R}\left[x_{1}, \ldots, x_{n}\right]$ et $V$ l'ensemble $\mathcal{Z}(J)$. On note aussi $V_{\text {reg }}$ l'ensemble des points lisses de $V$. Alors

$$
\mathcal{Z}(I)=\overline{V_{\text {reg }}}
$$

est l'unique composante $C$-irréductible de dimension maximale d de $V$. De plus, si $W$ désigne la réunion des composantes $\mathcal{A} \mathcal{R}$-irréductibles de $V$ de dimension $d$, alors

$$
{\overline{V_{\text {reg }}}}^{\mathcal{A R}}=W \subseteq \mathcal{Z}(I) .
$$

Démonstration. D'après le Théorème 6.4, on sait déjà que $\mathcal{Z}(I)$ est un ensemble $C$-irréductible fermé, c'est-à-dire un fermé régulu irréductible. On décompose $V=\cup_{i=1}^{s} W_{i} \cup \cup_{j=1}^{t} Z_{j}$ en composantes $C$-irréductibles fermées où les $W_{i}$ sont de dimension maximale $d$ et $\operatorname{dim} Z_{j}<d$ pour $j \in\{1, \ldots, t\}$. D'après la Proposition 6.2, l'ensemble $\mathcal{Z}(I)$ est $C$-irréductible de dimension $d$. Par conséquent, on peut supposer que $\mathcal{Z}(I)=W_{1}$ et il existe $f_{1} \in \mathcal{R}^{0}\left(\mathbf{R}^{n}\right)$ 
telle que $W_{1}=\mathcal{Z}\left(f_{1}\right)$. La fonction $f_{1}$ s'annule en fait identiquement sur $V_{\text {reg }}$ (Proposition 6.6). On a donc

$$
V_{\text {reg }} \subset \mathcal{Z}\left(f_{1}\right)=\mathcal{Z}(I) .
$$

Si $s \geq 2$, on écrit $W_{2}=\mathcal{Z}\left(f_{2}\right)$ avec $f_{2} \in \mathcal{R}^{0}\left(\mathbf{R}^{n}\right)$ et de la même manière on obtient $V_{\text {reg }} \subset \mathcal{Z}\left(f_{2}\right)=W_{2}$. Donc $V_{\text {reg }} \subset W_{1} \cap W_{2}$ ce qui est impossible car $V_{\text {reg }}$ est de dimension $d$. On en déduit que $\mathcal{Z}(I)$ est l'unique composante $C$-irréductible de dimension maximale de $V$ et finalement que $\mathcal{Z}(I)={\overrightarrow{V_{\text {reg }}}}^{C}$.

Remarquons que l'adhérence symétrique par arcs $\overline{V_{\text {reg }}} \mathcal{A} \mathcal{R}$ est égale à $W$ d'après [26, Thm. 2.21, Lem. 2.22]. Comme $V_{\text {reg }} \subset \mathcal{Z}(I)$, on en tire ${\overline{V_{\text {reg }}}}^{\mathcal{A R}}=$ $W \subseteq \mathcal{Z}(I)$.

On poursuit l'étude des relations entre les fonctions régulues sur une variété réelle algébrique et celles sur la réunion de ses composantes $\mathcal{A} \mathcal{R}$-irréductibles de dimension maximale.

Proposition 6.8. Soit $V$ une sous-variété algébrique irréductible de $\mathbf{R}^{n}$. On note $W=\overline{V_{\text {reg }}} \mathcal{A} \mathcal{R}$ la réunion des composantes $\mathcal{A} \mathcal{R}$-irréductibles de dimension maximale de $V$. Alors

$$
\mathcal{I}_{\mathcal{R}^{0}}(W)=\mathcal{I}_{\mathcal{R}^{0}}\left(V_{\text {reg }}\right)
$$

est un idéal premier de $\mathcal{R}^{0}\left(\mathbf{R}^{n}\right)$.

Démonstration. Si $f \in \mathcal{I}_{\mathcal{R}^{0}}\left(V_{\text {reg }}\right)$ alors $f \in \mathcal{I}_{\mathcal{R}^{0}}(W)$ car $\mathcal{Z}(f)$ est un ensemble symétrique par arcs. On a donc $\mathcal{I}_{\mathcal{R}^{0}}(W)=\mathcal{I}_{\mathcal{R}^{0}}\left(V_{\text {reg }}\right)$.

Soient $f$ et $g$ deux fonctions régulues telles que le produit $f . g$ s'annule

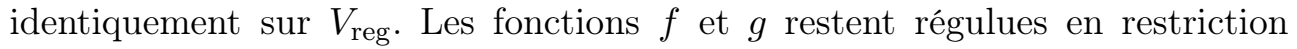
à $V$ d'après la Remarque 5.39, il existe donc une composition d'éclatements à centres lisses $\phi: \tilde{V} \rightarrow V$ telle que $f \circ \phi$ et $g \circ \phi$ soient régulières sur $\tilde{V}$, avec $\tilde{V}$ une variété réelle algébrique irréductible non singulière, d'après le Théorème 3.11. Le produit de fonctions régulières $(f \circ \phi) .(g \circ \phi)$ s'annule identiquement sur la variété irréductible $\tilde{V}$. On peut donc conclure que, par exemple, $f \circ \phi$ s'annule identiquement sur $\tilde{V}$ et donc $f$ s'annule identiquement sur $V_{\text {reg }}$ i.e. $f \in \mathcal{I}_{\mathcal{R}^{0}}\left(V_{\text {reg }}\right)$, ce qui termine la preuve.

Proposition 6.9. Soit $V$ une sous-variété algébrique irréductible de $\mathbf{R}^{n}$. On note $W=\overline{V_{\text {reg }}} \mathcal{A} \mathcal{R}$ la réunion des composantes $\mathcal{A} \mathcal{R}$-irréductibles de dimension maximale de $V$. Si $V=W$ (en particulier si $V$ est lisse), alors $V$ est un ensemble régulument fermé et régulument irréductible.

Démonstration. Comme les fonctions régulières sont régulues, $V$ est un fermé régulu. De plus $V=W$ donc l'idéal $\mathcal{I}_{\mathcal{R}^{0}}(W)=\mathcal{I}_{\mathcal{R}^{0}}(V)$ est un idéal premier par la Proposition 6.8.

Supposons que $V$ soit la réunion $V_{1} \cup V_{2}$ de deux fermés régulus non-vides. D'après le Théorème 5.21 , il existe des fonctions régulues $f_{1}, f_{2}$ telles que $V_{1}=\mathcal{Z}\left(f_{1}\right)$ et $V_{2}=\mathcal{Z}\left(f_{2}\right)$. Alors le produit $f_{1} . f_{2}$ appartient à l'idéal premier $\mathcal{I}_{\mathcal{R}^{0}}(V)$ et on en déduit que $V \subseteq V_{1}$ ou $V \subseteq V_{2}$. 
Soit $V \subset \mathbf{R}^{n}$ une sous-variété algébrique irréductible. On note $W=\overline{V_{\text {reg }}} \mathcal{A} \mathcal{R}$ la réunion des composantes $\mathcal{A} \mathcal{R}$-irréductibles de dimension maximale de $V$. La même preuve que celle de la proposition précédente montre que $W$ est un ensemble régulument irréductible. Mais ce n'est pas en général un ensemble régulument fermé.

Exemple 6.10. On considère la sous-variété algébrique irréductible $V$ de $\mathbf{R}^{4}=$ $\mathbf{R}^{2} \times \mathbf{R}^{2}$ de dimension 2 donnée par les équations

$$
\left\{\begin{array}{l}
(x+2)(x+1)(x-1)(x-2)+y^{2}=0 \\
u^{2}=x v^{2}
\end{array}\right.
$$

Cette variété est un ensemble régulument irréductible.
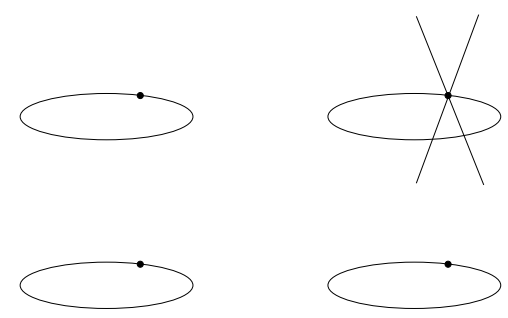

FiguRE 2. Un fermé régulument irréductible.

Sur la figure, on a représenté la fibre de $(x, y, u, v) \mapsto(x, y)$ au dessus de chaque point des deux composantes connexes de la courbe hyperelliptique d'équation $(x+2)(x+1)(x-1)(x-2)+y^{2}=0$. Au dessus de l'un des ovales la fibre est un point, alors qu'au dessus de l'autre ovale, la fibre est formée de deux droites sécantes.

La variété $V$ possède deux composantes connexes $W$ et $Z$, avec $W$ l'unique composante symétrique par arcs irréductible de dimension 2 de $V$. Le lieu singulier de $V$ est la courbe hyperelliptique $C$ d'équations $(x+2)(x+1)(x-$ 1) $(x-2)+y^{2}=0$ et $u=v=0$, possédant deux composantes connexes $Z$ et $Y$ avec $Y \subset W$. On suppose que $W$ est un fermé régulu. Il existe donc $f \in \mathcal{R}^{0}\left(\mathbf{R}^{4}\right)$ telle que $\mathcal{Z}(f)=W$ et par conséquent $f$ s'annule identiquement sur $Y$ mais pas sur $Z$. Une telle fonction ne peut exister par la Proposition 6.6 en considérant sa restriction à $C=Z \cup Y$.

Les résultats précédents permettent de déterminer la dimension de Krull de l'anneau $\mathcal{R}^{k}\left(\mathbf{R}^{n}\right)$ pour $k \in \mathbf{N}$.

Proposition 6.11. Soit $k \in \mathbf{N}$. L'espace topologique $\mathbf{R}^{n}$ est de dimension $n$ pour la topologie régulue. L'anneau $\mathcal{R}^{k}\left(\mathbf{R}^{n}\right)$ est par conséquent de dimension de Krull n.

Démonstration. Soit

$$
W_{0} \subsetneq W_{1} \subsetneq \ldots \subsetneq W_{m} \subseteq \mathbf{R}^{n}
$$

une suite d'inclusions strictes de fermés régulus irréductibles avec $m>n$. Pour $i=0, \ldots, m$, on note $I_{i}=\mathcal{I}_{\mathcal{R}^{0}}\left(W_{i}\right), J_{i}=I_{i} \cap \mathbf{R}\left[x_{1}, \ldots, x_{n}\right]$ et $V_{i}={\overline{W_{i}}}^{\text {Zar }}$. On a $\operatorname{dim} W_{i}=\operatorname{dim} V_{i}$ et de plus $V_{i}$ est irréductible pour la topologie de Zariski 
car $V_{i}=\mathcal{Z}\left(J_{i}\right)$ d'après la Proposition 6.2 (l'idéal $J_{i}$ est bien un idéal premier). On a aussi $W_{i}={\overline{\left(V_{i}\right)_{\text {reg }}}}^{C}$ d'après le Théorème 6.7. On obtient par conséquent une suite d'inclusions strictes de fermés de Zariski irréductibles

$$
V_{0} \subsetneq V_{1} \subsetneq \ldots \subsetneq V_{m} \subsetneq \mathbf{R}^{n}
$$

avec $m>n$, ce qui est impossible. La dimension régulue de $\mathbf{R}^{n}$ est donc inférieure à $n$. Il est clairement possible de construire une suite d'inclusions strictes

$$
V_{0} \subsetneq V_{1} \subsetneq \ldots \subsetneq V_{n}=\mathbf{R}^{n}
$$

de fermés de Zariski irréductibles lisses. D'après la Proposition 6.9, les $V_{i}$ sont des fermés régulus irréductibles ce qui prouve que la dimension régulue de $\mathbf{R}^{n}$ est bien $n$. Soit $k \in \mathbf{N}$. On rappelle que les topologies $k$-régulue et 0 régulues coincident. En utilisant le Nullstellensatz $k$-régulu, on en déduit que la dimension de Krull de $\mathcal{R}^{k}\left(\mathbf{R}^{n}\right)$ est $n$.

Exemples 6.12. On considère trois exemples (les deux premiers sont classiques).

- Le parapluie de Whitney :

Considérons la sous-variété $V$ d'équation $z x^{2}=y^{2}$ dans $\mathbf{R}^{3}$. Le parapluie $V$ est un ensemble régulument fermé et vérifie la relation $V={\overline{V_{\text {reg }}}}^{\mathcal{A}} \mathcal{R}$. Par la Proposition 6.9, c'est un ensemble régulument fermé et irréductible.

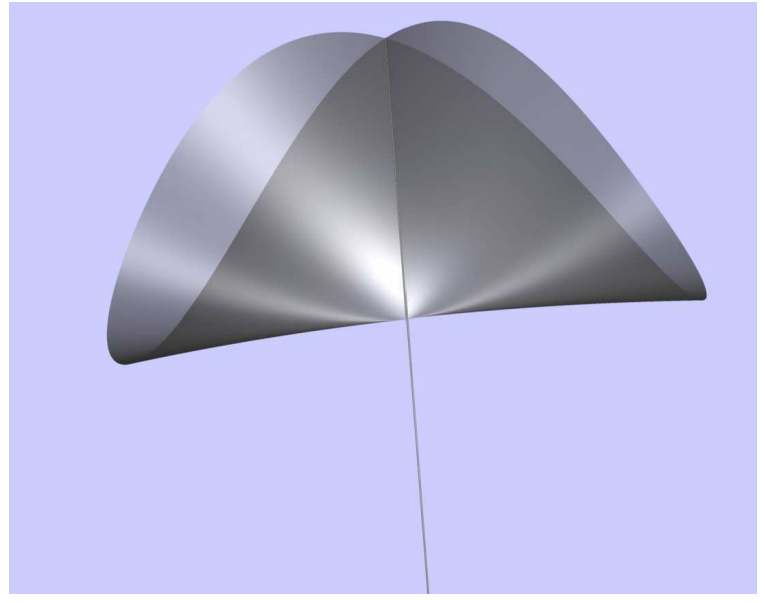

Figure 3. Parapluie de Whitney.

- Le parapluie de Cartan :

Considérons la sous-variété $V$ d'équation $z\left(x^{2}+y^{2}\right)=x^{3}$ de $\mathbf{R}^{3}$. La décomposition de $V$ en ensembles $\mathcal{A} \mathcal{R}$-irréductibles est $W \cup Z$ où $W={\overline{V_{\text {reg }}}}^{\mathcal{A} \mathcal{R}}$ est la toile du parapluie et $Z$ est le manche. L'ensemble $W$ est régulument fermé car $W=\mathcal{Z}(f)$ avec $f=z-\frac{x^{3}}{x^{2}+y^{2}}$. Par la Proposition 6.8, $W$ est régulument irréductible. L'ensemble $Z$ est un ensemble régulument fermé car $Z=\mathcal{Z}\left(x^{2}+y^{2}\right)$. L'ensemble $V$ est donc un ensemble régulument fermé et non-régulument irréductible. 


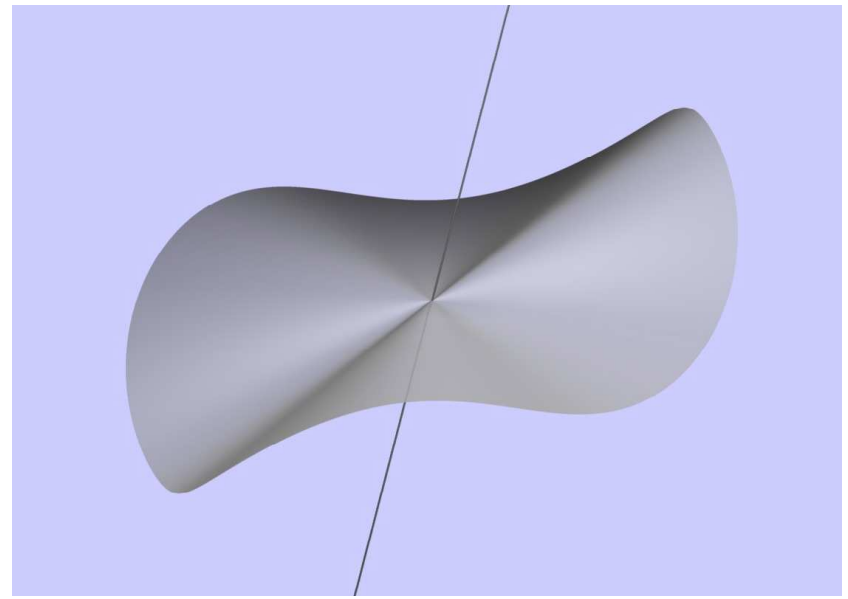

Figure 4. Parapluie de Cartan.

- Un parapluie cornu :

Considérons la sous-variété $V$ d'équation $s(x, y, z)=x^{2}+y^{2}\left(\left(y-z^{2}\right)^{2}+\right.$ $\left.y z^{3}\right)=x^{2}+y^{4}+y^{2} z^{4}+y^{3} z^{3}-2 y^{3} z^{2}=0$ de $\mathbf{R}^{3}$. La décomposition de $V$ en ensembles $\mathcal{A} \mathcal{R}$-irréductibles est $W \cup Z$ où $W={\overline{V_{\text {reg }}}}^{\mathcal{A R}}$ est la corne du parapluie et $Z$ est le manche. L'ensemble $W$ est régulument fermé car $W=\mathcal{Z}(f)$ avec $f=z^{2} \frac{s(x, y, z)}{x^{2}+y^{4}+y^{2} z^{4}}$. Par la Proposition $6.8, W$ est régulument irréductible. Le manche $Z$ est un ensemble régulument fermé car $Z=\mathcal{Z}\left(x^{2}+y^{2}\right)$. Comme pour le parapluie de Cartan, l'ensemble $V$ est donc un ensemble régulument fermé et non-régulument irréductible.

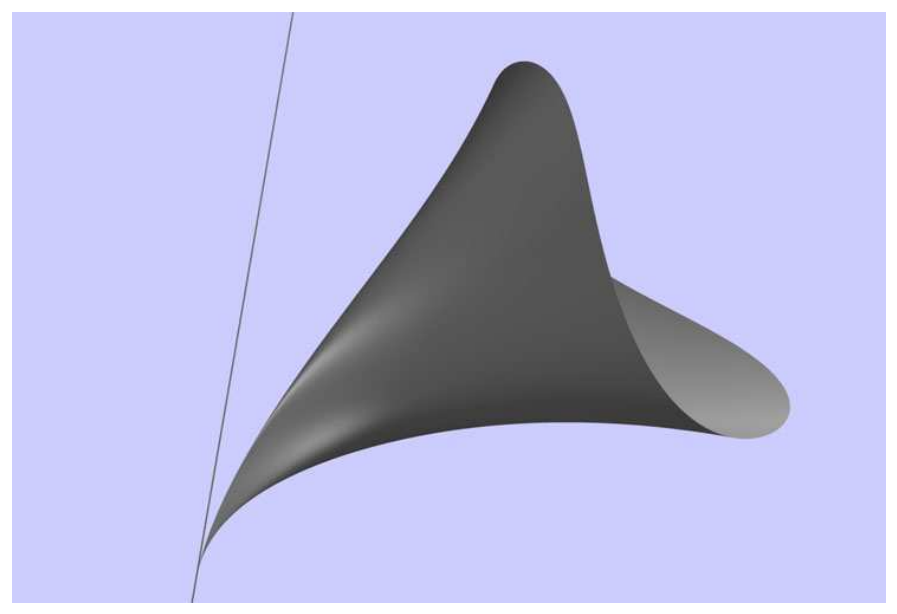

FiguRE 5. Un parapluie cornu.

On énonce maintenant un théorème qui résume ce qui précède et qui via le Nullstellensatz, est équivalent au Théorème 6.7. 
Théorème 6.13. Les fermés irréductibles régulus de $\mathbf{R}^{n}$ sont les ensembles du type

$$
\overline{V_{\text {reg }}} C
$$

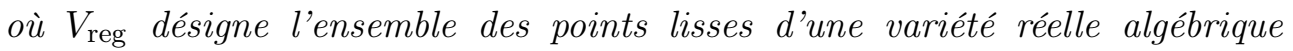
irréductible $V \subset \mathbf{R}^{n}$.

Le résultat suivant permet de construire de façon algorithmique le plus petit fermé algébriquement constructible contenant les points réguliers d'une variété réelle algébrique affine et donc de caractériser les fermés irréductibles régulus de $\mathbf{R}^{n}$.

Proposition 6.14. Soit $V \subset \mathbf{R}^{n}$ une sous-variété réelle algébrique irréductible. Soit $W$ la réunion des composantes symétriques par arcs de dimension maximale de $V$. Soit $Z$ une composante symétrique par arc irréductible de dimension strictement plus petite que $V$. Si

$$
\operatorname{dim} \bar{Z}^{Z a r} \cap W=\operatorname{dim} Z,
$$

alors $Z$ est dans $\bar{W}^{C}$.

Remarque 6.15. La réciproque de la Proposition 6.14 est fausse, voir l'Exemple 6.16 .

Démonstration de la Proposition 6.14. Notons que l'adhérence de Zariski de $Z$ forme une variété réelle algébrique irréductible de même dimension que $Z$. Soit $f$ une fonction régulue définie sur $\mathbf{R}^{n}$ et s'annulant sur $W$. Par hypothèse, la fonction $f$ s'annule sur un ensemble semi-algébrique $S=\bar{Z}^{Z a r} \cap W$ de même dimension que $\bar{Z}^{Z a r}$, donc sur $Z$ aussi d'après la Proposition 6.6. Par conséquent $Z$ appartient à l'adhérence pour la topologie algébriquement constructible de $W$.

On décrit maintenant de manière algorithmique l'unique composante algébriquement constructible irréductible de dimension maximale d'une variété réelle algébrique affine en termes d'ensembles symétriques par arcs irréductibles. Soit $V \subset \mathbf{R}^{n}$ une sous-variété réelle algébrique affine irréductible de dimension $d$. On note

$$
V=\cup_{i \in I_{d}} W_{i} \cup_{i=0}^{d-1} \cup_{j \in I_{i}} Z_{j}^{i}
$$

la décomposition de $V$ en sous-ensembles symétriques par arcs irréductibles, avec $\operatorname{dim} Z_{j}^{i}=i$ pour $j \in I_{i}$. On note $W$ la réunion des composantes symétriques par arcs de dimension maximale de $V$.

Soit $j \in I_{d-1}$.

(1) Si $Z_{j}^{d-1}$ satisfait la condition de la Proposition 6.14, alors $Z_{j}^{d-1} \subset \bar{W}^{C}$.

(2) Sinon, $Z_{j}^{d-1}$ n'est pas tout entier contenu dans $\bar{W}^{C}$. Néanmoins, un sous-ensemble symétrique par arcs de $Z_{j}^{d-1}$ peut être contenu dans $\bar{W}^{C}$. L'intersection $Z_{j}^{d-1} \cap W$ est un ensemble symétrique par arcs de dimension au plus $d-2$, et on rajoute les composantes symétriques par

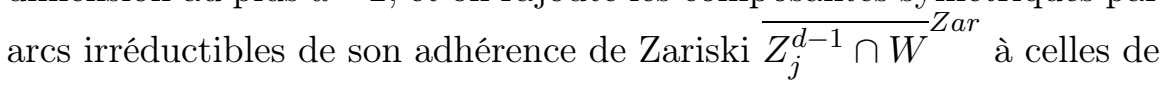


dimension au plus $d-2$ de $V$. Procédant de même pour tout $j \in I_{d-1}$, on obtient ainsi une réunion d'ensembles symétriques par arcs irréductibles de dimension au plus $d-2$

$$
\cup_{i=0}^{d-2} \cup_{j \in I_{i}^{2}} Z_{j}^{i}
$$

où $I_{i}^{2}$ est un ensemble d'indices fini contenant $I_{i}$.

Notons $W^{2}$ la réunion de $W$ et des composantes $Z_{j}^{d-1}$, pour $j \in I_{d-1}$, satisfaisant la condition de la Proposition 6.14. On renouvelle maintenant les opérations (1) et (2) avec $W^{2}$ à la place de $W$ et en considérant les indices $j$ appartenant à $I_{d-2}^{2}$. Ainsi, on construit pas à pas, en au plus $d-1$ étapes, l'adhérence pour la topologie algébriquement constructible de $W$.

Exemple 6.16. On modifie légèrement l'exemple 6.10 de la façon suivante. On considère la sous-variété algébrique irréductible $V^{\prime}$ de $\mathbf{R}^{6}$ de dimension 3 donnée par les équations

$$
\left\{\begin{array}{l}
(x+2)(x+1)(x-1)(x-2)(x-4)(x-5)+y^{2}=0 \\
w^{2}+u^{2}=x v^{2} \\
t^{2}=(x-3) w^{2}
\end{array}\right.
$$

Notons $C$ la courbe donnée par les équations de $V^{\prime}$ en faisant $t=u=v=$ $w=0$. La décomposition en sous-ensembles symétriques par arcs irréductibles de $V^{\prime}$ est de la forme $V^{\prime}=Z_{1}^{\prime} \cup Z_{2}^{\prime} \cup W^{\prime}$ avec $Z_{1}^{\prime}$ l'ovale de $C$ contenant le point de coordonnées $(-2,0,0,0,0,0), Z_{2}^{\prime}$ la surface contenant l'ovale de $C$ contenant le point de coordonnées $(1,0,0,0,0,0)$, et $W^{\prime}$ la partie de dimension trois contenant le dernier ovale de $C$.
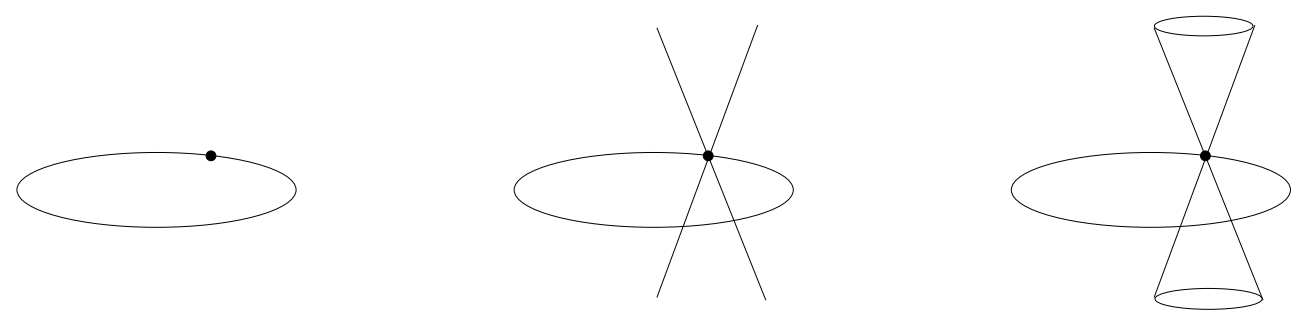

Figure 6. Une chaine d'ensembles symétriques par arcs.

Soit $T \in \mathbf{R}[x, y, t, u, v, w]$ l'équation d'un tore contenant en son intérieur l'ovale $C \cap W^{\prime}$ (c'est-à-dire l'ovale de droite sur le dessin). On suppose de plus que $T$ est négatif à l'intérieur du tore et positif à l'extérieur (cf. Example 3.2.8 dans [6]). On définit alors $V$ dans $\mathbf{R}^{7}$, en ajoutant une variable $s$, par les équations de $V^{\prime}$ auxquelles on rajoute l'équation $s^{2}=T$.

La variété $V$ ainsi obtenue forme un revêtement double de $V^{\prime}$ à l'extérieur du tore, et efface la partie à l'intérieur du tore. Notons $W$ la partie symétrique par arcs de dimension maximale de $V$. Soit $Z_{1}$ une composante symétrique par arcs de dimension un de $V$ s'envoyant sur l'ovale $Z_{1}^{\prime}$ (de gauche sur le dessin) de la courbe $C$ par le revêtement. L'adhérence de Zariski de $Z_{1}$, formée de quatre ovales vivant au dessus des ovales $Z_{1}^{\prime}$ et $Z_{2}^{\prime} \cap C$ de la courbe $C$, ne 
rencontre pas $W$ par construction de $V$. Ainsi $Z_{1}$ ne satisfait pas l'hypothèse de la Proposition 6.14. Pour autant, $Z_{1}$ est bien dans l'adhérence pour la topologie algébriquement constructible de $W$. En effet, les deux composantes symétriques par arcs de dimension deux de $V$ satisfont les hypothèses de la Proposition 6.14 puisque ${\overline{Z_{2}^{\prime}}}^{Z a r}=V^{\prime} \cap\{t=0\}$, et donc font partie de $\bar{W}^{C}$. Notons $Z_{2}$ leur réunion. Alors l'adhérence de Zariski de $Z_{1}$ rencontre $Z_{2}$ le long d'une courbe, donc d'après la Proposition $6.14, Z_{1}$ est dans l'adhérence pour la topologie algébriquement constructible de $Z_{2}$, donc dans celle de $W$.

\section{RÉFÉRENCES}

[1] A. Andreotti, E. Bombieri, Sugli omeomorfismi delle varietà algebriche, Ann. Scuola Norm. Sup Pisa (3) 23, 431-450, 1969.

[2] A. Andreotti, F. Norguet, La convexité holomorphe dans l'espace analytique des cycles d'une variété algébrique, Ann. Scuola Norm. Sup. Pisa (3) 21, 31-82, 1967.

[3] E. Bierstone, P. D. Milman, Arc-analytic functions, Invent. Math. 101, 411-424, 1990.

[4] I. Biswas, J. Huisman, Rational real algebraic models of topological surfaces, Doc. Math. 12, 549-567, 2007.

[5] J. Blanc, F. Mangolte, Geometrically rational real conic bundles and very transitive actions, Compositio Mathematica 147, 161-187, 2011.

[6] J. Bochnak, M. Coste, M.-F. Roy, Géométrie algébrique réelle, Ergeb. Math. Grenzgeb. 3 Folge, 12. Berlin Heidelberg New York, Springer 1987.

[7] M. Carral, M. Coste, Normal spectral spaces and their dimensions, Journal of Pure and Applied Algebra 30, 227-235, 1983.

[8] M. Coste, M. M. Diop, Real algebraic 1-cocycles are Nash coboundaries, Boll. Un. Mat. Ital. A (7) 6, no. 2, 249-254, 1992.

[9] C. N. Delzell, A continuous, constructive solution to Hilbert's 17th problem, Invent. Math. 76, 365-384, 1984.

[10] A. Grothendieck, J. Dieudonné, EGA1, Le langage des schémas, Publ. Math. IHES 4, 1960.

[11] A. Grothendieck, Éléments de géométrie algébrique. III. Étude cohomologique des faisceaux cohérents. I. Publ. Math. IHES 11, 1961.

[12] J. Harris, Algebraic Geometry, Graduate Texts in Mathematics 133, SpringerVerlag, 1992.

[13] R. Hartshorne, Algebraic geometry, Graduate Texts in Mathematics 52, SpringerVerlag, 1977.

[14] H. Hironaka, Resolution of singularities of an algebraic variety over a field of characteristic zero, Ann. Math. 79, 109-326, 1964.

[15] J. Huisman, F. Mangolte, The group of automorphisms of a real rational surface is n-transitive, Bull. London Math. Soc. 41, 563-568, 2009.

[16] S. Holzer, O. Labs, SuRfex 0.90. University of Mainz and University of Saarbrücken (2008) www.surfex.AlgebraicSurface.net

[17] J. H. Hubbard, On the cohomology of Nash sheaves, Topology 11, 265-270, 1972.

[18] J. Kollár, Lectures on resolution of singularities, Princeton University Press, 2007.

[19] J. Kollár, Continuous rational functions on real and p-adic varieties, arXiv:1101.3737 [math.AG].

[20] J. Kollár, K. Nowak, Continuous rational functions on real and p-adic varieties II, arXiv:1301.5048 [math.AG]. 
[21] J. Kollár, F. Mangolte, Cremona transformations and diffeomorphisms of surfaces, Adv. in Math. 222, 44-61, 2009.

[22] W. Kucharz, Rational maps in real algebraic geometry, Adv. Geom. 9 (4), 517-539, 2009.

[23] T.-C. Kuo, On classification of real singularities, Invent. Math. 82, 257-262, 1985.

[24] K. Kurdyka, Ensemble semi-algébriques symétriques par arcs, Math. Ann. 282, 445462, 1988.

[25] K. Kurdyka, Injective endomorphisms of real algebraic sets are surjective, Math. Ann. 313, 69-82, 1999.

[26] K. Kurdyka, A. Parusiński, Arc-symmetric sets and arc-analytic mappings, Arc spaces and additive invariants in real algebraic and analytic geometry, 33-67, Panor. Synthèses 24, Soc. Math. France, Paris, 2007.

[27] K. J. Nowak, On the Euler characteristic of the links of a set determined by smooth definable functions, Ann. Polon. Math. 93, no. 3, 231-246, 2008.

[28] A. Parusiński, Topology of injective endomorphisms of real algebraic sets, Math. Ann. 328, no. 1-2, 353-372, 2004.

[29] W. Rudin, Principles of mathematical analysis, International student editions, 1976.

[30] J-P. Serre, Faisceaux algébriques cohérents, Ann. of Math. (2) 61, 197-278, 1955.

[31] M. Spivak, Calculus, Publish or Perish, Houston, 1984.

[32] G. Stengle, A Nullstellensatz and a Positivstellensatz in semialgebraic geometry, Math. Ann. 207, 87-97, 1974.

Goulwen Fichou, IRMAR (UMR 6625), Université de Rennes 1, Campus de Beaulieu, 35042 Rennes Cedex, France

E-mail address: goulwen.fichou@univ-rennes1.fr

Johannes Huisman, LMBA (UMR 6205), Université de Bretagne Occidentale, 6, Av. Victor Le Gorgeu, CS 93837, 29238 Brest Cedex 3, France

E-mail address: Johannes.Huisman@univ-brest.fr

Frédéric Mangolte, LUnAM Université, LAREMA, Université d'Angers

E-mail address: frederic.mangolte@univ-angers.fr

JeAn-Philippe Monnier, LUnAM Université, LAREMA, Université D'Angers

E-mail address: jean-philippe.monnier@univ-angers.fr 